\title{
Construction of triangulated categories of motives using the localization property
}

\author{
Doosung Park
}

\begin{abstract}
Using the localization property, we construct a triangulated category of motives over quasiprojective $T$-schemes for any coefficient where $T$ is a noetherian separated scheme, and we prove the Grothendieck six operations formalism. We also construct integral étale realization of motives.
\end{abstract}

\section{Introduction}

1.1. Throughout this paper, fix a ring $\Lambda$, and fix a noetherian separated scheme $T$. Let

(1) $\mathscr{S}\left(\right.$ resp. $\left.\mathscr{S}^{s m}\right)$ denote the category of schemes quasi-projective (resp. quasi-projective and smooth) over $T$,

(2) $\operatorname{Tri}^{\otimes}$ denote the 2-category of symmetric monoidal triangulated categories where 1-morphisms are symmetric monoidal functors and 2-morphisms are symmetric monoidal natural transformations,

(3) Sm denote the class of smooth morphisms of schemes.

1.2. According to Ayo07, when $\Lambda$ is a $\mathbb{Q}$-algebra, we have triangulated categories of motives over schemes satisfying the Grothendieck six operations formalism in CD12, 2.4.50]. However, for general $\Lambda$, such a construction have not been available yet according to our knowledge.

1.3. In this paper, we define $\mathrm{DM}^{l o c}$, which is a successful construction of triangulated categories of motives over quasi-projective $T$-schemes with all coefficients satisfying the Grothendieck six operations formalism. Our idea is as follows. For any object $S$ of $\mathscr{S}^{s m}$, the construction $\operatorname{DM}(S, \Lambda)$ in [CD12, 11.1.1] seems to enjoy many useful properties, so put

$$
\operatorname{DM}^{l o c}(S, \Lambda)=\operatorname{DM}(S, \Lambda)
$$

for such an $S$.

Assume that we can extend the above $\operatorname{DM}^{l o c}(-, \Lambda)$ to $\mathscr{S}$ such that the Grothendieck six operations formalism is satisfied. Let $i: Z \rightarrow S$ be a closed immersion in $\mathscr{S}$, and let $j: U \rightarrow S$ denote its complement. Then from the formalism, $i_{*}$ should be fully faithful, and we should have the distinguished triangle

$$
j_{\sharp} j^{*} \longrightarrow \mathrm{id} \longrightarrow i_{*} i^{*} \longrightarrow j_{\sharp} j^{*}[1] .
$$

Thus we see that $\operatorname{DM}^{l o c}(Z, \Lambda)$ should be the full subcategory of $\operatorname{DM}^{l o c}(S, \Lambda)$ consisting of objects $K$ of $\mathrm{DM}^{l o c}(S, \Lambda)$ such that $j^{*} K=0$. 
We can consider it as our definition of triangulated categories of motives. A problem is that the definition itself is dependent on the choice of the closed immersion $i: Z \rightarrow S$, so we need to show that the definition is independent of the choice of $i$. We also need to show that $\operatorname{DM}^{l o c}(-, \Lambda)$ satisfies the Grothendieck six operations formalism. We solve these problems by proving the following theorems.

Theorem 1.4. (7.4) in the text) Consider a diagram

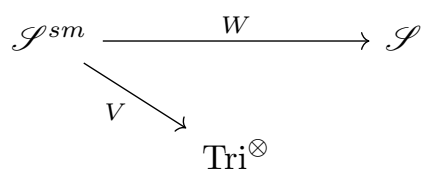

of 2 -categories where

(i) $V$ is a Sm-premotivic pseudofunctor satisfying (Loc) (see (2.6) and (4.7) for the definitions),

(ii) $W$ denotes the inclusion functor.

Then there is a contravariant pseudofunctor $H: \mathscr{S} \rightarrow \mathrm{Tri}^{\otimes}$ satisfying (Loc) and a pseudonatural equivalence $\kappa: V \rightarrow H \circ W$ making the above diagram commutes.

The above construction is functorial in the following sense. Suppose that we have a diagram

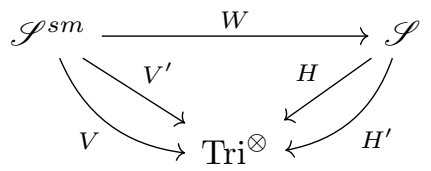

of 2-categories and a diagram

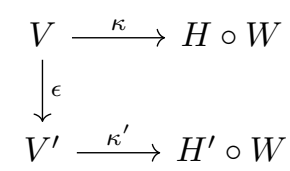

of pseudofunctors where $V^{\prime}$ and $H^{\prime}$ are Sm-premotivic pseudofunctors satisfying (Loc), $\kappa^{\prime}$ is a pseudonatural equivalence, and $\epsilon$ is a Sm-premotivic pseudonatural transformation (see (2.9) for the definition). Then there exists a Sm-premotivic pseudonatural transformation $H \rightarrow H^{\prime}$ unique up to isomorphism such that the induced Sm-pseudonatural transformation $H \circ W \rightarrow H^{\prime} \circ W$ makes the diagram (1.4.1) commutative.

1.5. Note that in (1.4), the functoriality implies that $H$ is unique up to pseudonatural equivalence.

Theorem 1.6. ((8.2) in the text) In (1.4), if $V$ satisfies the axioms from (B-5) to (B-8) in (2.10), then $H$ satisfies the Grothendieck six operations formalism in [CD12, 2.4.50], which is as follows.

(1) For any morphism $f: X \rightarrow S$ in $\mathscr{S}$, there exists 3 pairs of adjoint functors as follows:

$$
\begin{gathered}
f^{*}: H(S) \rightleftarrows H(X): f_{*}, \\
f_{!}: H(X) \rightleftarrows H(S): f^{!},
\end{gathered}
$$

$(\otimes, H o m)$, symmetric closed monoidal structure on $H(S)$. 
(2) There exists a structure of covariant (resp. contravariant) pseudofunctors on $f \mapsto f_{*}, f \mapsto f_{\text {: }}$ (resp. $f \mapsto f^{*}, f \mapsto f^{!}$).

(3) There exists a canonical natural transformation

$$
\alpha_{f}: f_{!} \rightarrow f_{*}
$$

which is an isomorphism when $f$ is proper.

(4) For any smooth separated morphism $f: X \rightarrow S$ in $\mathscr{S}$ of relative dimension d with tangent bundle $T_{f}$, there exists a canonical natural isomorphism

$$
f^{!} \otimes \operatorname{MTh}\left(-T_{f}\right) \longrightarrow f^{*}
$$

See [CD12, 2.4.12] for the definition of MTh.

If $H$ admits an orientation in the sense of [CD12, 2.4.38] and $f$ has dimension d, then there exists a canonical natural isomorphism

$$
f^{!}(-d)[-2 d] \rightarrow f^{*}
$$

(5) For any Cartesian diagram

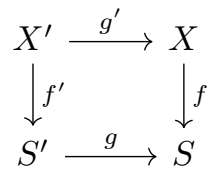

in $\mathscr{S}$, there exist natural isomorphisms

$$
\begin{aligned}
& g^{*} f_{!} \stackrel{\sim}{\rightarrow} f_{!}^{\prime} g^{*}, \\
& g_{*}^{\prime} f^{\prime !} \stackrel{\sim}{\rightarrow} f^{!} g_{*} .
\end{aligned}
$$

(6) For any morphism $f: X \rightarrow S$ in $\mathscr{S}$, there exist natural isomorphisms

$$
\begin{aligned}
& f_{!} K \otimes_{S} L \stackrel{\sim}{\longrightarrow} f_{!}\left(K \otimes_{X} L\right), \\
& \operatorname{Hom}_{S}\left(f_{!}(L), K\right) \stackrel{\sim}{\longrightarrow} f_{*} \operatorname{Hom}_{X}\left(L, f^{!} K\right), \\
& f^{!} \operatorname{Hom}_{S}(L, M) \stackrel{\sim}{\longrightarrow} \operatorname{Hom}_{X}\left(f^{*} L, f^{!} M\right) .
\end{aligned}
$$

(7) For any closed immersion $i: Z \rightarrow S$ with complement $j: U \rightarrow S$, there exists a distinguished triangle of natural transformations

$$
j ! j^{!} \stackrel{a d^{\prime}}{\longrightarrow} \text { id } \stackrel{a d}{\longrightarrow} i_{*} i^{*} \stackrel{\partial_{i}}{\longrightarrow} j ! j^{!}[1]
$$

where ad (resp. ad') denotes the unit (resp. counit) of the relevant adjunction. 
1.7. Our primary application of the theorems is as follows. Consider the diagram

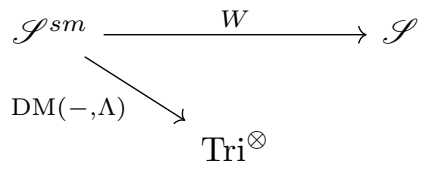

of 2-categories. In (9.1), we will check that $\operatorname{DM}(-, \Lambda): \mathscr{S}^{s m} \rightarrow \operatorname{Tr}^{\otimes}$ is a $S m$-premotivic pseudofunctor satisfying (Loc) and the axioms from (B-5) to (B-8), so by (1.5), there is a pseudofunctor $\operatorname{DM}^{l o c}(-, \Lambda): \mathscr{S} \rightarrow \operatorname{Tri}^{\otimes}$ unique up to pseudonatural equivalence such that it is an extension of $\operatorname{DM}(-, \Lambda): \mathscr{S}^{s m} \rightarrow \operatorname{Tr}^{\otimes}$ and satisfies the Grothendieck six operations formalism.

We can also apply the theorem to étale realization. Let $n$ be a positive integer, and assume that $T$ is a noetherian scheme separated over Spec $\mathbb{Z}[1 / n]$. In (9.5), we will construct the étale realization of $\operatorname{DM}(-, \mathbb{Z}): \mathscr{S}^{s m} \rightarrow \operatorname{Tr}^{\otimes}$, which is a $S m$-premotivic pseudonatural transformation

$$
R_{\grave{e} t, n}: \mathrm{DM}(-, \mathbb{Z}) \rightarrow \mathrm{D}_{\grave{e} t}(-, \mathbb{Z} / n \mathbb{Z}) .
$$

From this, we have the commutative diagram

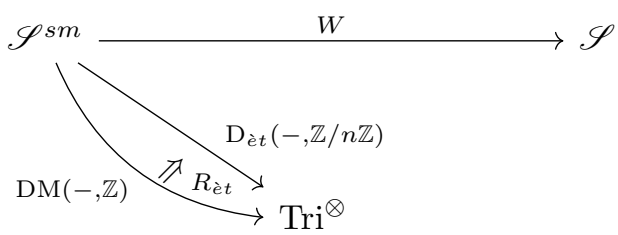

of 2-categories. Then by (1.4), there is a $S m$-premotivic pseudonatural transformation

$$
R_{\grave{e ̀ t}, n}^{l o c}: \mathrm{DM}^{l o c}(-, \mathbb{Z}) \rightarrow \mathrm{D}_{\grave{e} t}(-, \mathbb{Z} / n \mathbb{Z})
$$

unique up to isomorphism such that it is an extension of $R_{\grave{e} t, n}: \mathrm{DM}(-, \mathbb{Z}) \rightarrow \mathrm{D}_{\grave{e} t}(-, \mathbb{Z} / n \mathbb{Z})$.

1.8. We also prove that $\mathrm{DM}^{l o c}(-, \Lambda)$ admits an orientation, so the second part of (1.6(4)) holds for $\mathrm{DM}^{l o c}(-, \Lambda)$.

1.9. Organization of the paper. In Section 2, we review pseudofunctors, pseudonatural transformations, and modifications to help the reader to understand our notations. Then we review $\mathscr{P}$-premotivic pseudofunctors and $\mathscr{P}$-premotivic pseudonatural transformations. In Section 4, we define categories with immersions, and we review the localization property and motivic pseudofunctors. We also construct a category $\widetilde{\mathcal{C}}$ that will be used later.

In Sections 3, 5, 6, and 7, we prove the main theorem (1.4) by constructing the commutative diagram

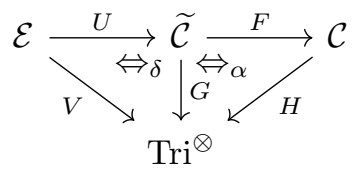

of 2-categories where $\mathcal{C}=\mathscr{S}$ and $\mathcal{E}=\mathscr{S}^{s m}$. In Section 3, we construct $H$ and $\alpha$ when $G$ is given and some conditions are satisfied. In Section 5 , we construct $G$ and $\delta$. In Section 6 , we show a 
condition for $F$ so that we can use the argument in Section 3. In Section 7, we show that $H$ is $S m$-premotivic, and then we prove the main theorem (1.4).

In Section 8, we prove (1.6), and in Section 9, we apply (1.4) and (1.6) to construct $\operatorname{DM}^{l o c}(-, \Lambda)$ and its étale realization. We also construct a pseudonatural equivalence between $\operatorname{DM}^{l o c}(-, \Lambda)$ and $\mathrm{DA}_{\grave{e} t}(-, \Lambda)$ when $\Lambda$ is a $\mathbf{Q}$-algebra. In Section 10, we prove that $\mathrm{DM}^{l o c}(-, \Lambda)$ admits an orientation.

1.10. Terminology and conventions. Alongside (1.1), we have the following notations. When we have an adjunction

$$
F: \mathcal{C} \leftrightarrows \mathcal{D}: G
$$

where $F$ and $G$ are contravariant functors of categories, we denote by $a d$ (resp. $a d^{\prime}$ ) the unit id $\longrightarrow G F$ (resp. the counit $F G \longrightarrow$ id).

1.11. Acknowledgements. The author is grateful to Martin Olsson for helpful conversations. The author is grateful to Adeel Khan for indicating that orientation for $\operatorname{DM}^{l o c}(-, \Lambda)$ is needed. Section 10 is obtained from a conversation with him.

\section{Premotivic pseudofunctors}

2.1. To help the reader to understand our notations, we include the definitions of pseudofunctors, pseudonatural transformations, and modifications as follows.

Definition 2.2. Let $\mathcal{C}$ be a category, and let $\mathcal{D}$ be a 2-category. A contravariant pseudofunctor $F: \mathcal{C} \rightarrow \mathcal{D}$ is the data of

(1) an object $F\left(S_{1}\right)$ of $\mathcal{D}$ for each object $S_{1}$ of $\mathcal{C}$,

(2) a 1-morphism

$$
F\left(f_{1}\right): F\left(S_{1}\right) \rightarrow F\left(S_{2}\right)
$$

in $\mathcal{D}$ for each morphism $f_{1}: S_{2} \rightarrow S_{1}$ in $\mathcal{C}$,

(3) an invertible 2-morphism

$$
F_{\operatorname{id}_{S_{1}}}: F\left(\mathrm{id}_{S_{1}}\right) \rightarrow \mathrm{id}
$$

in $\mathcal{D}$ for each object $S_{1}$ of $\mathcal{C}$,

(4) an invertible 2-morphism

$$
F_{f_{1}, f_{2}}: F\left(f_{2}\right) F\left(f_{1}\right) \rightarrow F\left(f_{1} f_{2}\right)
$$

in $\mathcal{D}$ for each pair of morphisms $f_{1}: S_{2} \rightarrow S_{1}$ and $f_{2}: S_{3} \rightarrow S_{2}$ in $\mathcal{C}$

satisfying the following axioms.

(i) For any morphism $f_{1}: S_{2} \rightarrow S_{1}$ in $\mathcal{C}$, the diagram

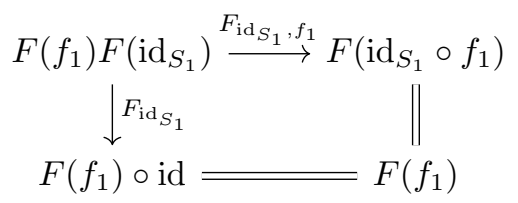

in $\mathcal{D}$ commutes. 
(ii) For any morphism $f_{1}: S_{2} \rightarrow S_{1}$ in $\mathcal{C}$, the diagram

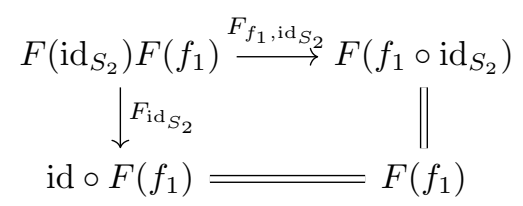

in $\mathcal{D}$ commutes.

(iii) For any morphisms $S_{4} \stackrel{f_{3}}{\rightarrow} S_{3} \stackrel{f_{2}}{\rightarrow} S_{2} \stackrel{f_{1}}{\rightarrow} S_{1}$ in $\mathcal{C}$, the diagram

$$
\begin{array}{rr}
F\left(f_{3}\right) F\left(f_{2}\right) F\left(f_{1}\right) \stackrel{F_{f_{1}, f_{2}}}{\longrightarrow} & F\left(f_{3}\right) F\left(f_{1} f_{2}\right) \\
\downarrow & \downarrow F_{f_{2}, f_{3}} \\
F\left(f_{2} f_{3}\right) F\left(f_{1}\right) \stackrel{F_{f_{1}, f_{2} f_{3}}}{\longrightarrow} & \downarrow F_{f_{1} f_{2}, f_{3}} \\
& F\left(f_{1} f_{2} f_{3}\right)
\end{array}
$$

commutes.

We often write $f_{1}^{*}$ for $F\left(f_{1}\right)$ when no confusion seems likely to arise.

Definition 2.3. Let $\mathcal{C}$ be a category, let $\mathcal{D}$ be a 2-category, and let $F, F^{\prime}: \mathcal{C} \rightarrow \mathcal{D}$ be contravariant pseudofunctors. A pseudonatural transformation $\alpha: F \rightarrow F^{\prime}$ is the data of

(1) 1-morphism

$$
\alpha\left(S_{1}\right): F\left(S_{1}\right) \rightarrow F^{\prime}\left(S_{1}\right)
$$

in $\mathcal{D}$ for each object $S_{1}$ of $\mathcal{C}$,

(2) invertible 2-morphism

$$
\alpha_{f_{1}}: \alpha\left(S_{2}\right) F\left(f_{1}\right) \rightarrow F^{\prime}\left(f_{1}\right) \alpha\left(S_{1}\right)
$$

in $\mathcal{D}$ for each morphism $f_{1}: S_{2} \rightarrow S_{1}$ in $\mathcal{C}$

satisfying the following axioms.

(i) For any object $S_{1}$ of $\mathcal{C}$, the diagram

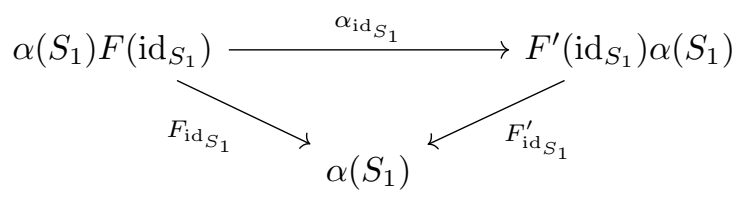

in $\mathcal{D}$ commutes.

(ii) For any morphisms $S_{3} \stackrel{f_{2}}{\rightarrow} S_{2} \stackrel{f_{1}}{\rightarrow} S_{1}$ in $\mathcal{C}$, the diagram

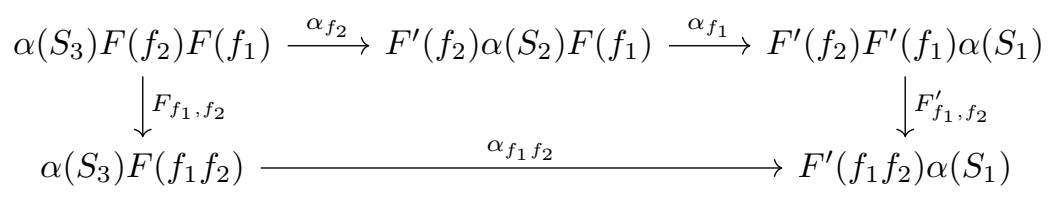

in $\mathcal{D}$ commutes. 
A pseudonatural transformation $\alpha: F \rightarrow F^{\prime}$ is called a pseudonatural equivalence if $\alpha\left(S_{1}\right)$ is invertible for any object $S_{1}$ of $\mathcal{C}$.

Definition 2.4. Let $\mathcal{C}$ be a category, let $\mathcal{D}$ be a 2-category, let $F, F^{\prime}: \mathcal{C} \rightarrow \mathcal{D}$ be contravariant pseudofunctors, and let $\alpha, \alpha^{\prime}: F \rightarrow F^{\prime}$ be pseudonatural transformations. A modification $\Phi: \alpha \rightarrow$ $\alpha^{\prime}$ is the data of 2-morphism

$$
\Phi_{S_{1}}: \alpha\left(S_{1}\right) \rightarrow \alpha^{\prime}\left(S_{1}\right)
$$

for each object $S_{1}$ of $\mathcal{C}$ satisfying the axiom that for any morphism $f_{1}: S_{2} \rightarrow S_{1}$ in $\mathcal{C}$, the diagram

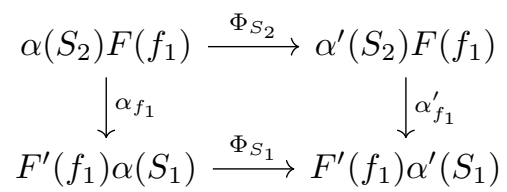

in $\mathcal{D}$ commutes.

A modification $\Phi: \alpha \rightarrow \alpha^{\prime}$ is called an isomorphism if $\Phi_{S_{1}}$ is invertible for any object $S_{1}$ of $\mathcal{C}$.

2.5. Now, following [CD12, 1.4.2, 1.4.6], we will define $\mathscr{P}$-premotivic pseudofunctors and $\mathscr{P}$ premotivic pseudonatural transformations as follows.

Definition 2.6. Let $\mathcal{C}$ be a category with fiber products, and let $\mathscr{P}$ be a class of morphisms of $\mathcal{C}$ containing all isomorphisms and stable by compositions and pullbacks. We say that a pseudofunctor

$$
H: \mathcal{C} \longrightarrow \operatorname{Tr}^{\otimes}
$$

is $\mathscr{P}$-premotivic if it satisfies the following axioms.

(B-1) For any morphism $f$ in $\mathcal{C}$, the functor $f^{*}:=H(f)$ has a right adjoint, denoted by $f_{*}$.

(B-2) For any morphism $f$ in $\mathscr{P}$, the functor $f^{*}$ has a left adjoint, denoted by $f_{\sharp}$.

(B-3) For any Cartesian diagram

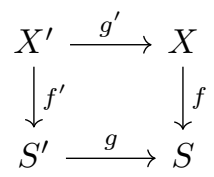

in $\mathcal{C}$ with $f \in \mathscr{P}$, the natural transformation

$$
E x: f_{\sharp} g^{*} \longrightarrow g^{\prime *} f_{\sharp}^{\prime}
$$

given by the composition

$$
f_{\sharp} g^{*} \stackrel{a d}{\longrightarrow} f_{\sharp} g^{*} f^{\prime *} f_{\sharp}^{\prime} \stackrel{\sim}{\longrightarrow} f_{\sharp} f^{*} g^{\prime *} f_{\sharp}^{\prime} \stackrel{a d^{\prime}}{\longrightarrow} g^{\prime *} f_{\sharp}^{\prime}
$$

is an isomorphism. The natural transformation (2.6.1) is called an exchange transformation. 
(B-4) For any morphism $f: X \rightarrow S$ in $\mathscr{P}$, and for any objects $K$ of $H(X)$ and $L$ of $H(S)$, the natural transformation

$$
E x: f_{\sharp}\left(K \otimes_{X} f^{*} L\right) \longrightarrow f_{\sharp} K \otimes_{S} L
$$

given by the composition

$$
f_{\sharp}\left(K \otimes_{X} f^{*} L\right) \stackrel{a d}{\longrightarrow} f_{\sharp}\left(f^{*} f_{\sharp} K \otimes_{X} f^{*} L\right) \stackrel{\sim}{\longrightarrow} f_{\sharp} f^{*}\left(f_{\sharp} K \otimes_{S} L\right) \stackrel{a d^{\prime}}{\longrightarrow} f_{\sharp} K \otimes_{S} L
$$

is an isomorphism. Here, the second arrow is obtained by the fact that $f^{*}$ is monoidal. The natural transformation (2.6.2) is called an exchange transformation.

Remark 2.7. In (2.6), note that if $f$ is an isomorphism, then the axioms from (B-1) to (B-4) for $f$ is always satisfied.

Definition 2.8. Under the notations and hypotheses of (2.6), assume that $H$ is $\mathscr{P}$-premotivic. For any object $S$ of $\mathcal{C}$, we denote by $1_{S}$ the unit of $H(S)$ obtained by the monoidal structure of $H(S)$.

Definition 2.9. Let $\mathcal{C}$ be a category with fiber products, and let $\mathscr{P}$ be a class of morphisms of $\mathcal{C}$ containing all isomorphisms and stable by compositions and pullbacks, and let $H, H^{\prime}: \mathcal{C} \rightarrow \operatorname{Tri}^{\otimes}$ be $\mathscr{P}$-premotivic pseudofunctors. We say that a pseudonatural transformation

$$
\alpha: H \rightarrow H^{\prime}
$$

is $\mathscr{P}$-premotivic if it satisfies the following axioms.

(C-1) For any object $S_{1}$ of $\mathcal{C}$, the functor

$$
\alpha\left(S_{1}\right): H\left(S_{1}\right) \rightarrow H^{\prime}\left(S_{1}\right)
$$

has a right adjoint.

(C-2) For any morphism $f_{1}: S_{2} \rightarrow S_{1}$ in $\mathscr{P}$, the natural transformation

$$
E x: f_{1 \sharp} \alpha\left(S_{2}\right) \stackrel{a d}{\longrightarrow} f_{1 \sharp} \alpha\left(S_{2}\right) f_{1}^{*} f_{1 \sharp} \stackrel{\alpha_{f_{1}}}{\longrightarrow} f_{1 \sharp} f_{1}^{*} \alpha\left(S_{1}\right) f_{1 \sharp} \stackrel{a d^{\prime}}{\longrightarrow} \alpha\left(S_{1}\right) f_{1 \sharp}
$$

is an isomorphism.

Remark 2.10. In (2.9), note that if $f_{1}$ is an isomorphism, then the axiom (C-2) for $f_{1}$ is always satisfied.

Proposition 2.11. Under the notations and hypotheses of (2.9), if $\alpha\left(S_{1}\right)$ is an isomorphism for any object $S_{1}$ of $\mathcal{C}$, then $H$ is $\mathscr{P}$-premotivic.

Proof. For any object $S_{1}$ of $\mathcal{C}$, let $\beta\left(S_{1}\right)$ denote the inverse of $\alpha\left(S_{1}\right)$. Then $\beta\left(S_{1}\right)$ is both the right adjoint and left adjoint of $\alpha\left(S_{1}\right)$, so $(\mathrm{C}-1)$ is satisfied.

Let $f_{1}: S_{2} \rightarrow S_{1}$ be a morphism in $\mathcal{P}$. The left adjoint of the isomorphism

$$
\alpha_{f_{1}}: \alpha\left(S_{2}\right) f_{1}^{*} \longrightarrow f_{1}^{*} \alpha\left(S_{1}\right)
$$

is the composition

$$
\begin{aligned}
\beta\left(S_{1}\right) f_{1 \sharp} & \stackrel{a d}{\longrightarrow} \beta\left(S_{1}\right) f_{1 \sharp} \alpha\left(S_{2}\right) \beta\left(S_{2}\right) \stackrel{a d}{\longrightarrow} \beta\left(S_{1}\right) f_{1 \sharp} \alpha\left(S_{2}\right) f_{1}^{*} f_{1 \sharp \beta(} \beta\left(S_{2}\right) \\
& \stackrel{\alpha_{f_{1}}}{\longrightarrow} \beta\left(S_{1}\right) f_{1 \sharp} f_{1}^{*} \alpha\left(S_{1}\right) f_{1 \sharp} \beta\left(S_{2}\right) \stackrel{a d^{\prime}}{\longrightarrow} \beta\left(S_{1}\right) \alpha\left(S_{1}\right) f_{1 \sharp} \beta\left(S_{2}\right) \stackrel{a d^{\prime}}{\longrightarrow} f_{1 \sharp} \beta\left(S_{2}\right),
\end{aligned}
$$


which is also an isomorphism. The first and fifth arrows are isomorphisms since $\beta\left(S_{1}\right)$ and $\beta\left(S_{2}\right)$ are the inverses of $\alpha\left(S_{1}\right)$ and $\alpha\left(S_{2}\right)$ respectively. Thus the composition of the second, third, and fourth arrows is an isomorphism, applying $\alpha\left(S_{1}\right)$ to the left and $\alpha\left(S_{2}\right)$ to the right of the composition, we get the natural transformation in the axiom $(\mathrm{C}-2)$. Thus $H$ satisfies $(\mathrm{C}-2)$.

Remark 2.12. For example, the pseudonatural equivalence $\kappa$ in (1.4) is $S m$-premotivic.

Proposition 2.13. Let $\mathcal{C}$ and $\mathcal{D}$ be categories with fiber product, and let $\mathscr{P}$ be a class of morphisms in $\mathcal{C}$ containing all isomorphisms and stable by compositions and pullback. Consider a commutative diagram

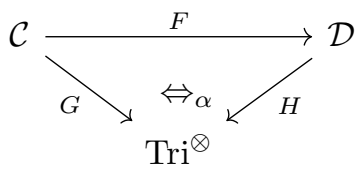

of 2-categories where

(i) F is a covariant functor preserving fiber products,

(ii) $G$ and $H$ are contravariant pseudofunctors,

(iii) $\alpha$ is a pseudonatural equivalence.

Then $G$ is $\mathscr{P}$-premotivic if and only if $H$ is $F(\mathscr{P})$-premotivic.

Proof. It follows from (2.7) since every morphism in $F(\mathscr{P})$ is a composition of isomorphisms and morphisms of the form $F(f)$ for $f \in \mathscr{P}$.

Proposition 2.14. Under the notations and hypotheses of (2.13), consider a commutative diagram

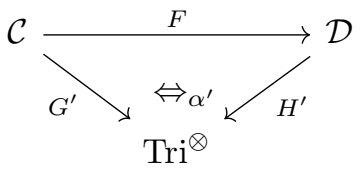

of 2-categories and a diagram

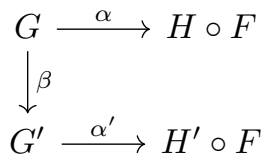

of pseudofunctors where

(i) $G^{\prime}$ is a $\mathscr{P}$-premotivic pseudofunctor and $H^{\prime}$ are contravariant pseudofunctors,

(ii) $\alpha^{\prime}$ is a pseudonatural equivalence.

(iii) $\beta$ is a $\mathscr{P}$-premotivic pseudonatural transformation.

If $\gamma: H \rightarrow H^{\prime}$ is a pseudonatural transformation such that the induced pseudonatural transformation $H \circ F \rightarrow H^{\prime} \circ F$ makes the above diagram commutative, then $\gamma$ is $F(\mathscr{P})$-premotivic.

Proof. It follows from (2.10) since every morphism in $F(\mathscr{P})$ is a composition of isomorphisms and morphisms of the form $F(f)$ for $f \in \mathscr{P}$. 


\section{Proof of (1.4), part I}

3.1. Throughout this section, we fix a diagram

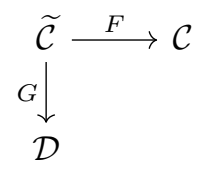

where

(i) $\mathcal{C}$ and $\widetilde{\mathcal{C}}$ are categories, and $\mathcal{D}$ is a 2-category,

(ii) $F$ is an essentially surjective covariant functor, and $G$ is a contravariant pseudofunctor.

We denote by $\mathscr{W}$ the class of morphisms in $\widetilde{\mathcal{C}}$ such that $f \in \mathscr{W}$ if and only if $F(f)$ is an isomorphism. Assume that for any morphism $f$ in $\mathscr{W}, f^{*}$ is invertible.

Consider the category $\mathcal{C}^{\prime}$ where ob $\mathcal{C}^{\prime}:=\mathrm{ob} \widetilde{\mathcal{C}}$ and

$$
\operatorname{Hom}_{\mathcal{C}^{\prime}}\left(X_{1}, X_{2}\right)=\operatorname{Hom}_{\mathcal{C}}\left(F\left(X_{1}\right), F\left(X_{2}\right)\right)
$$

for any $X_{1}, X_{2} \in$ ob $\mathcal{C}^{\prime}$. Then $\mathcal{C}^{\prime}$ is equivalent to $\mathcal{C}$ since $F$ is essentially surjective, so it is not harmful to consider $\mathcal{C}^{\prime}$ instead of $\mathcal{C}$. Thus we may assume ob $\mathcal{C}:=$ ob $\widetilde{\mathcal{C}}$.

Under this assumption, for any object $X$ of $\mathcal{C}$, we denote by $\widetilde{X}$ the corresponding object in $\widetilde{C}$ to avoid confusion.

3.2. We will study when the diagram (3.1.1) can be extended to a commutative diagram

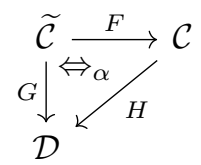

of 2-categories where $H$ is a contravariant pseudofunctor and $\alpha: G \rightarrow H \circ F$ is a pseudonatural equivalence. Consider the following conditions for $G$.

(A-1) For any morphism $X_{2} \stackrel{f_{1}}{\rightarrow} X_{1}$ in $\mathcal{C}$, we can choose a diagram

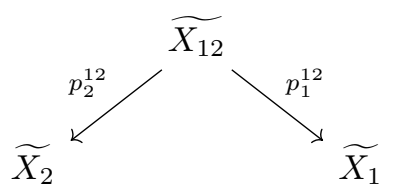

in $\widetilde{\mathcal{C}}$ such that $p_{2}^{12}$ is in $\mathscr{W}$ and $f_{1}=F\left(p_{1}^{12}\right)\left(F\left(p_{2}^{12}\right)\right)^{-1}$. Moreover, when $f_{1}=F\left(\widetilde{f}_{1}\right)$ for some morphism $\widetilde{f}_{1}: \widetilde{X}_{2} \rightarrow \widetilde{X_{1}}$ in $\widetilde{\mathcal{C}}$, we require that $p_{2}^{12}$ has a section $d_{12}^{2}$ such that $p_{1}^{12} d_{12}^{2}=\widetilde{f}_{1}$. Here, $\widetilde{X_{1}}=X_{1}$ and $\widetilde{X}_{2}=X_{2}$, but we distinguish the notations following (3.1) to avoid confusion. 
(A-2) Assume $(\mathrm{A}-1)$. For any morphisms $X_{3} \stackrel{f_{2}}{\rightarrow} X_{2} \stackrel{f_{1}}{\rightarrow} X_{1}$ in $\mathcal{C}$, we can choose a commutative diagram

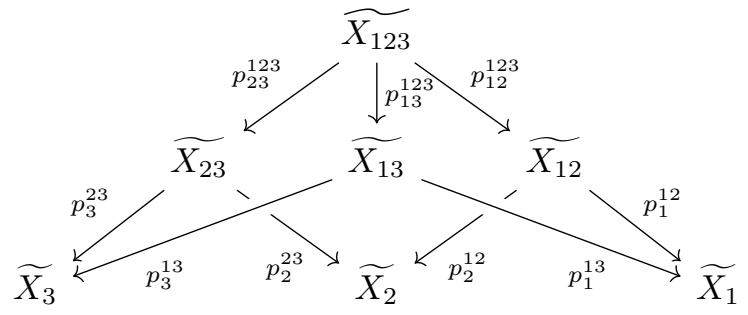

such that $p_{13}^{123}$ and $p_{23}^{123}$ are in $\mathscr{W}$. Here, $\widetilde{X_{12}}, \widetilde{X_{13}}, \widetilde{X_{23}}, p_{1}^{12}, \ldots, p_{3}^{23}$ are already chosen from $\left(\right.$ A-1). Moreover, when $f_{1}=F\left(\widetilde{f}_{1}\right)$ (resp. $f_{2}=F\left(\widetilde{f}_{2}\right)$ ) for some morphism $\widetilde{f}_{1}: \widetilde{X}_{2} \rightarrow \widetilde{X_{1}}$ (resp. $\widetilde{f}_{2}: \widetilde{X_{3}} \rightarrow \widetilde{X_{2}}$ ) in $\widetilde{\mathcal{C}}$, we require that $p_{23}^{123}$ (resp. $p_{13}^{123}$ ) has a section $d_{123}^{23}$ (resp. $d_{123}^{13}$ ) such that $p_{12}^{123} d_{123}^{23}=d_{12}^{2} p_{2}^{23}$ (resp. $p_{23}^{123} d_{123}^{13}=d_{23}^{3} p_{3}^{13}$ ). Here, $d_{12}^{2}$ and $d_{23}^{3}$ are defined in $(\mathrm{A}-1)$.

(A-3) Assume (A-1) and (A-2). For any morphisms $X_{4} \stackrel{f_{3}}{\rightarrow} X_{3} \stackrel{f_{2}}{\rightarrow} X_{2} \stackrel{f_{1}}{\rightarrow} X_{1}$ in $\mathcal{C}$, we can choose a commutative diagram

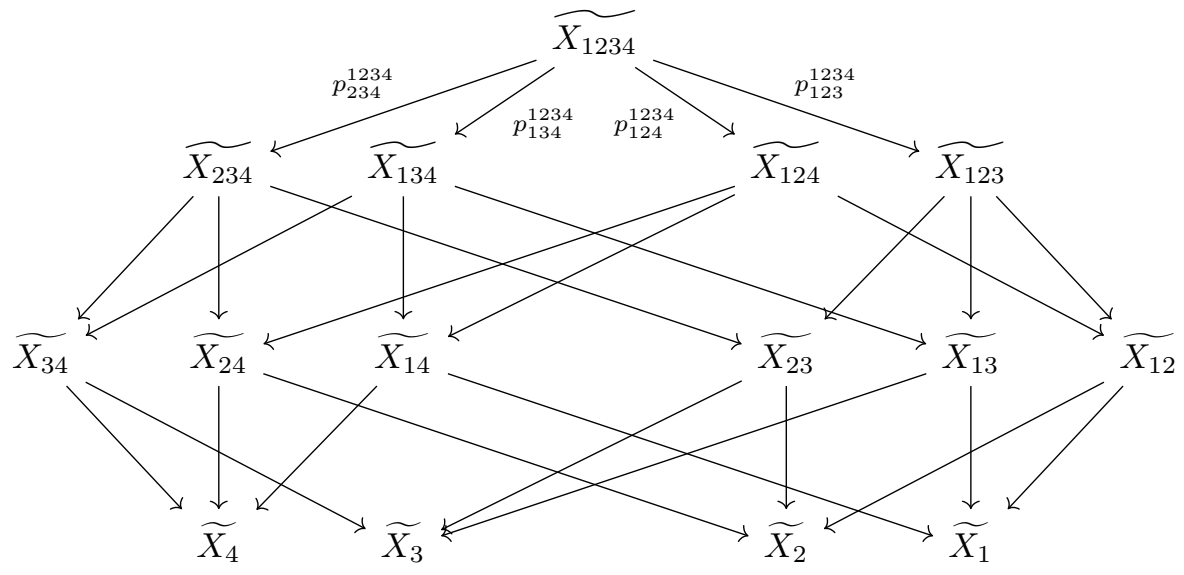

in $\widetilde{\mathcal{C}}$ such that $p_{124}^{1234}, p_{134}^{1234}$, and $p_{234}^{1234}$ are in $\mathscr{W}$. Here, $\widetilde{X_{12}}, \ldots, \widetilde{X_{34}}, \widetilde{X_{123}}, \ldots, \widetilde{X_{234}}$ and not denoted arrows are already chosen from $(\mathrm{A}-1)$ and $(\mathrm{A}-2)$.

3.3. Under the conditions from (A-1) to (A-3), we can construct a pseudofunctor $H$ as follows.

(1) For any object $X_{1}$ of $\mathcal{C}$, put

$$
H\left(X_{1}\right):=G\left(\widetilde{X_{1}}\right) .
$$

(2) For any morphism $f_{1}: X_{2} \rightarrow X_{1}$ in $\mathcal{C}$, we can fix a diagram

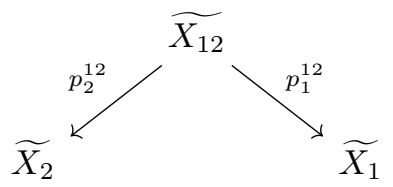


in $\widetilde{\mathcal{C}}$ by $(\mathrm{A}-1)$. Then put

$$
H\left(f_{1}\right):=\left(p_{2}^{12 *}\right)^{-1} p_{1}^{12 *} .
$$

(3) Under the notations and hypotheses of (2), if $f_{1}$ is the identity morphism, we have the invertible 2-morphism

$$
H_{f_{1}}: H\left(f_{1}\right) \longrightarrow \mathrm{id}
$$

given by

$$
\left(p_{2}^{12 *}\right)^{-1} p_{1}^{12 *} \stackrel{\sim}{\longrightarrow} d_{12}^{2 *} p_{2}^{12 *}\left(p_{2}^{12 *}\right)^{-1} p_{1}^{12 *} \stackrel{\sim}{\longrightarrow} d_{12}^{2 *} p_{1}^{12 *} \stackrel{\sim}{\longrightarrow} \mathrm{id}^{*} \stackrel{\sim}{\longrightarrow} \mathrm{id} .
$$

(4) For any morphisms $f_{2}: X_{3} \rightarrow X_{2}$ and $f_{1}: X_{2} \rightarrow X_{1}$ in $\mathcal{C}$, we have the invertible 2-morphism

$$
H_{f_{1}, f_{2}}: H\left(f_{2}\right) H\left(f_{1}\right) \longrightarrow H\left(f_{1} f_{2}\right)
$$

given by

$$
\begin{aligned}
\left(p_{3}^{23 *}\right)^{-1} p_{2}^{23 *}\left(p_{2}^{12 *}\right)^{-1} p_{1}^{12 *} & \stackrel{\sim}{\longrightarrow}\left(p_{3}^{23 *}\right)^{-1}\left(p_{23}^{123 *}\right)^{-1} p_{12}^{123 *} p_{1}^{12 *} \stackrel{\sim}{\longrightarrow}\left(p_{3}^{123 *}\right)^{-1} p_{1}^{123 *} \\
& \stackrel{\sim}{\longrightarrow}\left(p_{3}^{13 *}\right)^{-1}\left(p_{13}^{123 *}\right)^{-1} p_{13}^{123 *} p_{1}^{13 *} \stackrel{\sim}{\longrightarrow}\left(p_{3}^{13 *}\right)^{-1} p_{1}^{13 *} .
\end{aligned}
$$

where $p_{1}^{123}=p_{1}^{12} p_{12}^{123}$ and $p_{3}^{123}=p_{3}^{23} p_{23}^{123}$. Here, the notations are from (A-2), and the first arrow is induced by the 2-morphism

$$
p_{23}^{123 *} p_{2}^{23 *} \stackrel{\sim}{\longrightarrow} p_{12}^{123 *} p_{2}^{12 *} .
$$

We will verify the axioms of pseudofunctors for $H$ in (3.5).

3.4. Let us first study the functoriality of the condition (A-2). Assume the conditions from (A-1) to $(\mathrm{A}-3)$, and consider a commutative diagram

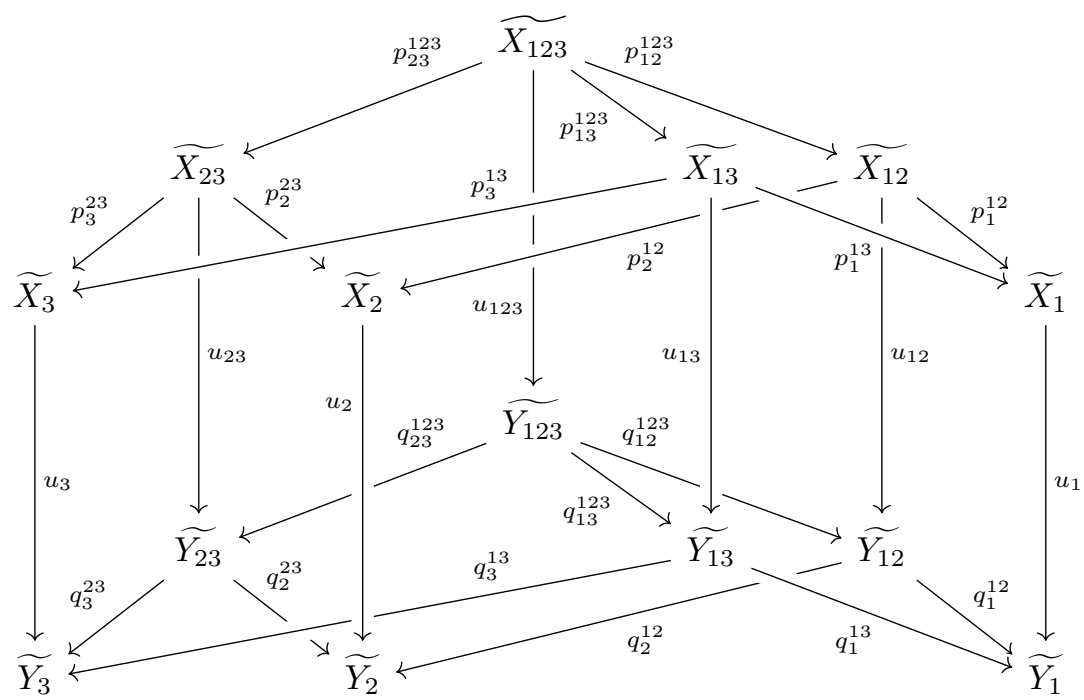


in $\widetilde{\mathcal{C}}$ such that $q_{2}^{12}, q_{3}^{13}, q_{3}^{23}, q_{13}^{123}, q_{23}^{123}$ are also in $\mathscr{W}$. Temporary put

$$
\begin{gathered}
P\left(f_{1}\right):=\left(q_{2}^{12 *}\right)^{-1} q_{1}^{12 *} \\
P\left(f_{2}\right):=\left(q_{3}^{23 *}\right)^{-1} q_{2}^{23 *} \\
P\left(f_{1} f_{2}\right):=\left(q_{3}^{13 *}\right)^{-1} q_{1}^{13 *} .
\end{gathered}
$$

We have the invertible 2-morphism

$$
P_{f_{1}, f_{2}}: P\left(f_{2}\right) P\left(f_{1}\right) \longrightarrow P\left(f_{1} f_{2}\right)
$$

in $\mathcal{D}$ as in (3.3), and we have the invertible 2-morphism

$$
E x: H\left(f_{1}\right) u_{1}^{*} \longrightarrow u_{2}^{*} P\left(f_{1}\right)
$$

in $\mathcal{D}$ given by

$$
\left(p_{2}^{12 *}\right)^{-1} p_{1}^{12 *} u_{1}^{*} \stackrel{\sim}{\longrightarrow}\left(p_{2}^{12 *}\right)^{-1} u_{12}^{*} q_{1}^{12 *} \stackrel{\sim}{\longrightarrow} u_{2}^{*}\left(q_{2}^{12 *}\right)^{-1} q_{1}^{12 *} .
$$

Similarly, we have the invertible 2-morphisms

$$
\begin{gathered}
H\left(f_{2}\right) u_{2}^{*} \stackrel{E x}{\longrightarrow} u_{3}^{*} P\left(f_{2}\right), \\
H\left(f_{1} f_{2}\right) u_{1}^{*} \stackrel{E x}{\longrightarrow} u_{3}^{*} P\left(f_{1} f_{2}\right) .
\end{gathered}
$$

in $\mathcal{D}$. Now, we have the diagram

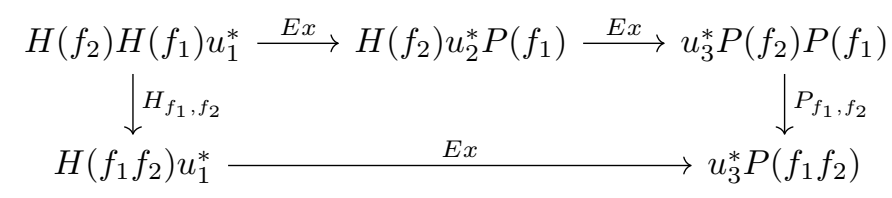

in $\mathcal{D}$. It is a big outside diagram of the diagram

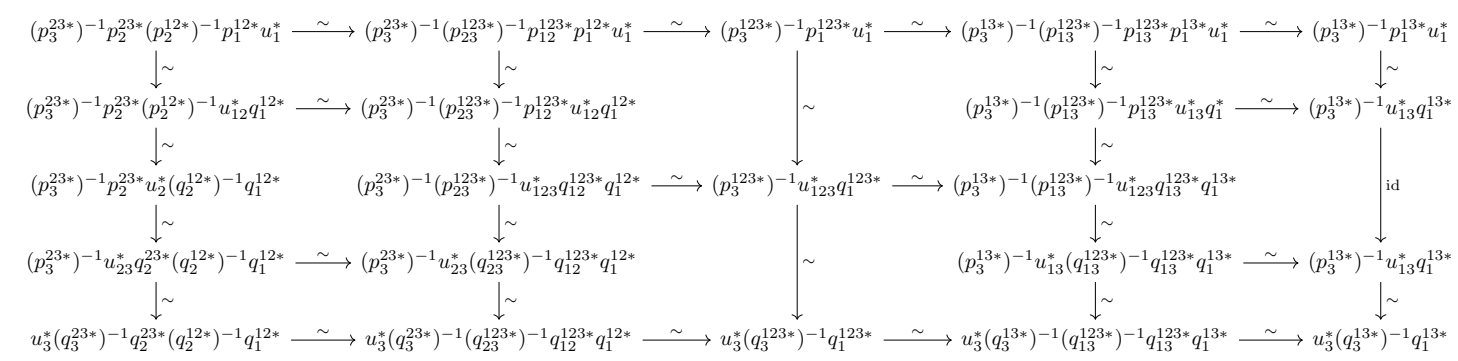

in $\mathcal{D}$, and each small square commutes since $G$ is a pseudofunctor. Thus (3.4.1) commutes.

3.5. Now, under the conditions from $(\mathrm{A}-1)$ to $(\mathrm{A}-3)$, we will show that $H$ constructed in (3.3) satisfies the axioms of pseudofunctors. 
(1) Let $X_{3} \stackrel{f_{2}}{\rightarrow} X_{2} \stackrel{f_{1}}{\rightarrow} X_{1}$ be morphisms in $\mathcal{C}$. When $f_{1}$ is the identity morphism, we have to show that the diagram

$$
\begin{gathered}
H\left(f_{2}\right) H\left(f_{1}\right) \stackrel{H_{f_{1}}}{\longrightarrow} H\left(f_{2}\right) \\
\downarrow H_{f_{1}, f_{2}}
\end{gathered}
$$

in $\mathcal{D}$ commutes. It follows from applying (3.4) to the commutative diagram

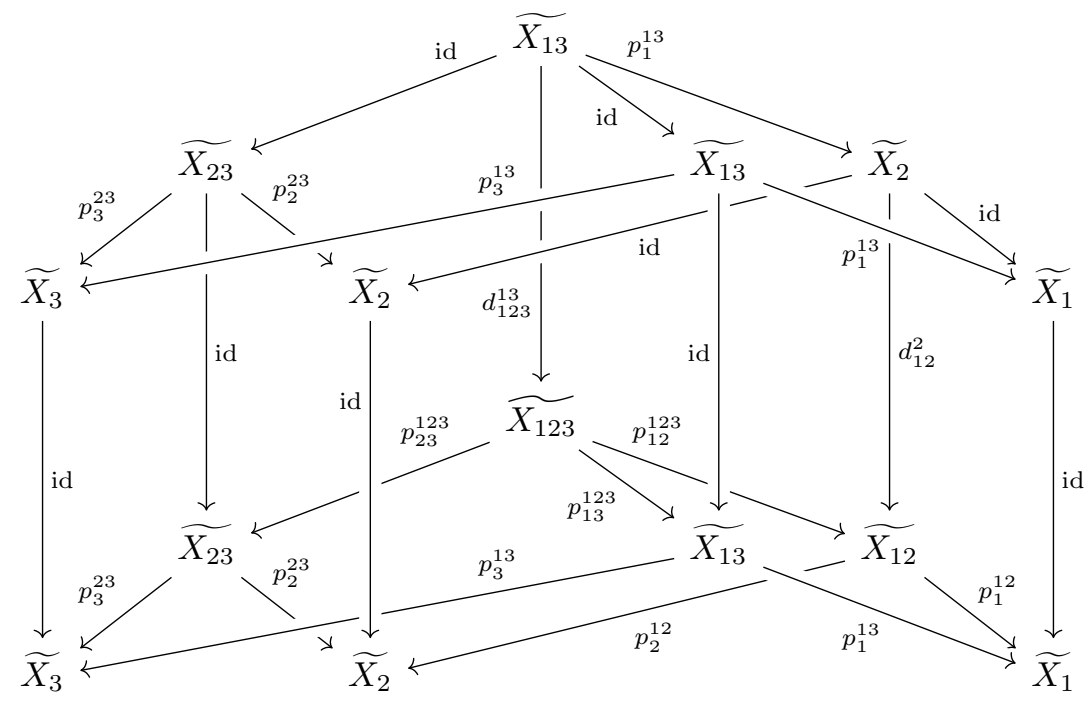

in $\widetilde{\mathcal{C}}$.

(2) Let $X_{3} \stackrel{f_{2}}{\rightarrow} X_{2} \stackrel{f_{1}}{\rightarrow} X_{1}$ be morphisms in $\mathcal{C}$. When $f_{2}$ is the identity morphism, as in (1), the diagram

$$
\begin{gathered}
H\left(f_{2}\right) H\left(f_{1}\right) \stackrel{H_{f_{2}}}{\longrightarrow} H\left(f_{1}\right) \\
\downarrow H_{f_{1}, f_{2}} \\
H\left(f_{1} f_{2}\right)=H\left(f_{1}\right)
\end{gathered}
$$

in $\mathcal{D}$ commutes. 
(3) Let $X_{4} \stackrel{f_{3}}{\rightarrow} X_{3} \stackrel{f_{2}}{\rightarrow} X_{2} \stackrel{f_{1}}{\rightarrow} X_{1}$ be morphisms in $\mathcal{C}$. Consider the commutative diagram

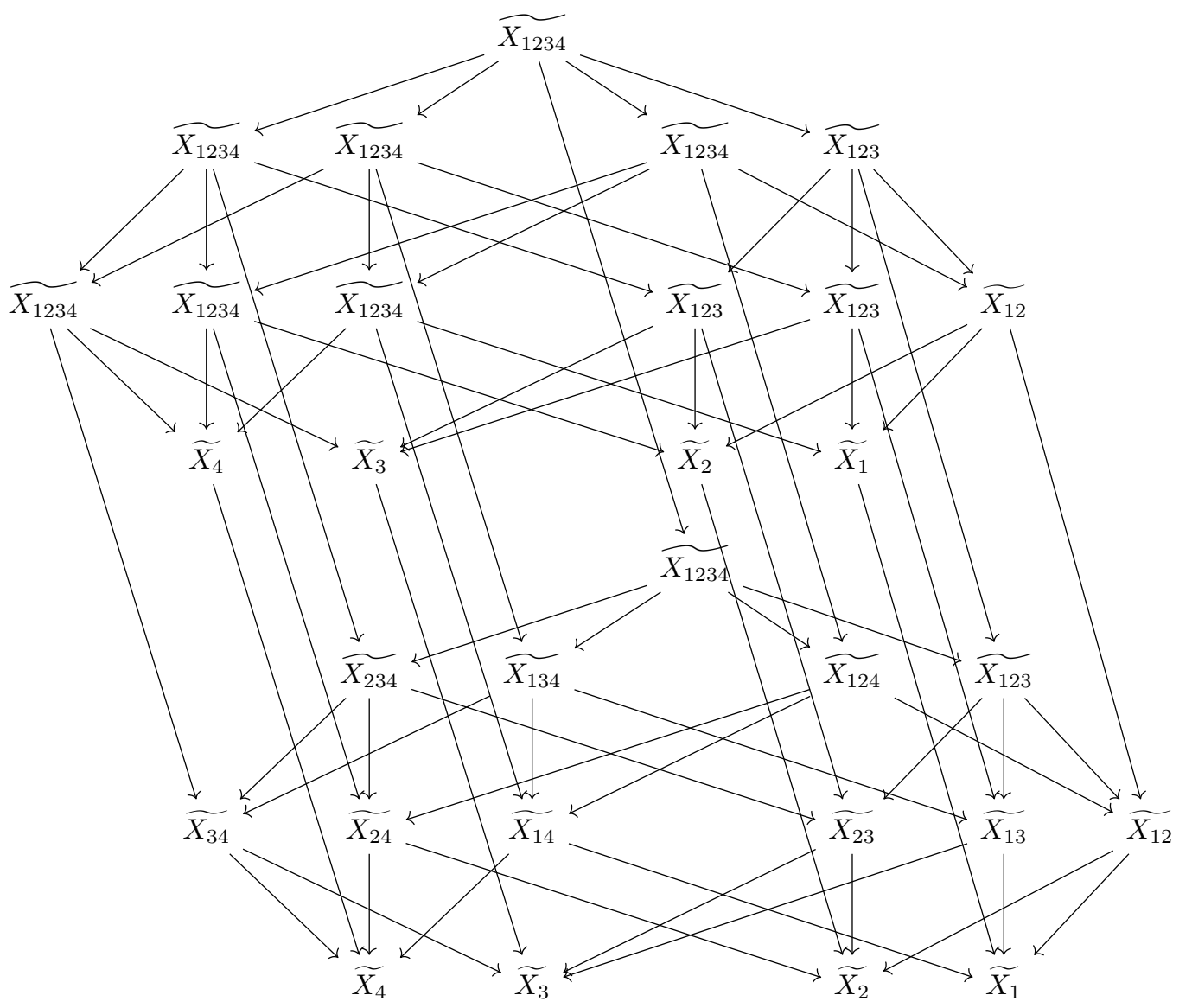

in $\mathcal{C}$. Here, each arrow is the identity morphism or a composition of morphisms in the diagram in $(\mathrm{A}-3)$. Let

$$
Q\left(f_{1}\right), Q\left(f_{2}\right), Q\left(f_{3}\right), Q\left(f_{1} f_{2}\right), Q\left(f_{1} f_{3}\right), Q\left(f_{1} f_{2} f_{3}\right), Q_{f_{1}, f_{2}}, Q_{f_{2}, f_{3}} \cdot Q_{f_{1} f_{2}, f_{3}}, Q_{f_{1}, f_{2} f_{3}}
$$

denote the 1-morphisms and 2-morphisms obtained as in (3.3) using the upper part of the above diagram instead of the diagram in (A-3). As in (3.4), we also have the invertible 2-morphisms

$$
\begin{gathered}
Q\left(f_{1}\right) \stackrel{E x}{\longrightarrow} H\left(f_{1}\right), \quad Q\left(f_{2}\right) \stackrel{E x}{\longrightarrow} H\left(f_{2}\right), \quad Q\left(f_{3}\right) \stackrel{E x}{\longrightarrow} H\left(f_{3}\right), \\
Q\left(f_{1} f_{2}\right) \stackrel{E x}{\longrightarrow} H\left(f_{1} f_{2}\right), \quad Q\left(f_{2} f_{3}\right) \stackrel{E x}{\longrightarrow} H\left(f_{2} f_{3}\right), \quad Q\left(f_{1} f_{2} f_{3}\right) \stackrel{E x}{\longrightarrow} H\left(f_{1} f_{2} f_{3}\right)
\end{gathered}
$$


in $\mathcal{D}$. Now applying (loc. cit), we see that in the diagram

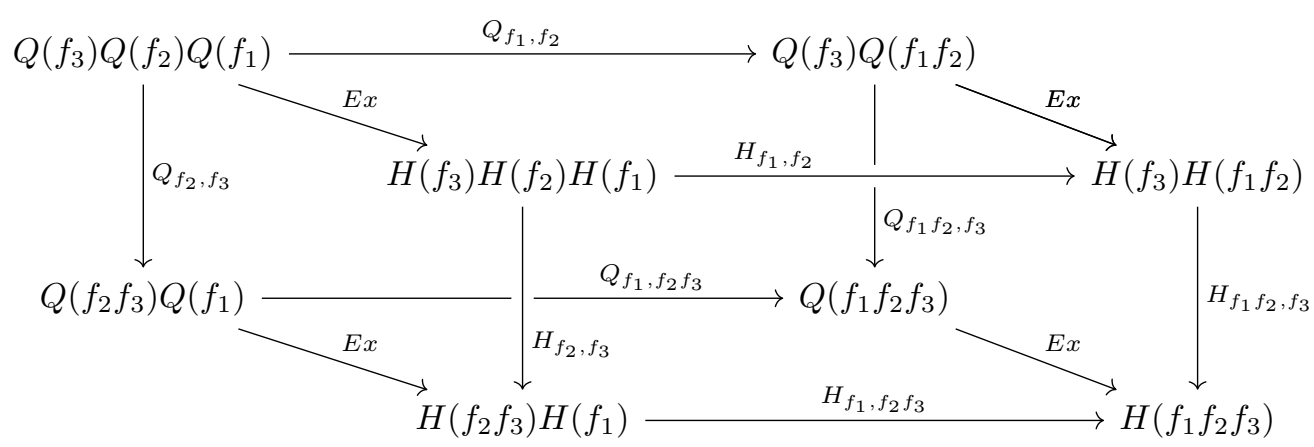

in $\mathcal{D}$, the small squares other than the front side and back side squares commute. Since the purpose is to show that the front side square commutes, we only need to show that the back side square commutes. Put

$$
p_{2}^{123}=p_{2}^{12} p_{12}^{123}, \quad p_{3}^{123}=p_{3}^{23} p_{23}^{123}, \quad p_{3}^{1234}=p_{3}^{123} p_{123}^{1234}, \quad p_{4}^{1234}=p_{4}^{34} p_{34}^{234} p_{234}^{1234},
$$

and consider the commutative diagram

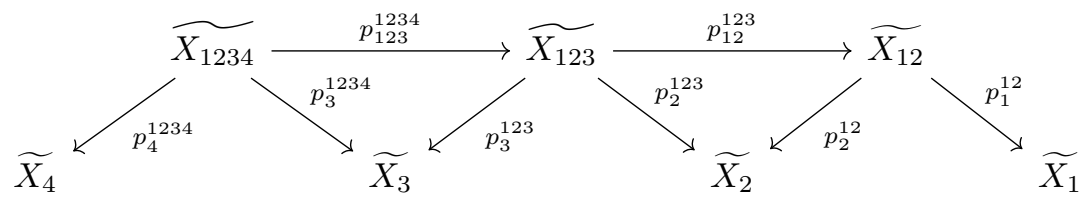

in $\widetilde{\mathcal{C}}$. The back side square is then the diagram

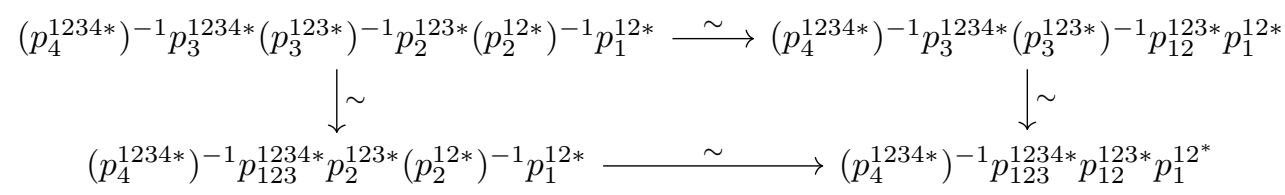

in $\mathcal{D}$, and it commutes since $G$ is a pseudofunctor.

We have proved all axioms of pseudofunctors for $H$, so $H$ is a pseudofunctor.

3.6. The remaining part to construct (3.2.1) is the construction of $\alpha: G \rightarrow H \circ F$. Under the conditions from $(\mathrm{A}-1)$ to $(\mathrm{A}-3)$, we can construct $\alpha$ as follows. For any object $\widetilde{X_{1}}$ of $\widetilde{\mathcal{C}}$, put $\alpha\left(\widetilde{X_{1}}\right)$ as the identity 1-morphism

$$
G\left(\widetilde{X_{1}}\right) \rightarrow(H \circ F)\left(\widetilde{X_{1}}\right) .
$$

For any morphism $\widetilde{X_{2}} \stackrel{\widetilde{f_{1}}}{\rightarrow} \widetilde{X_{1}}$ in $\widetilde{C}$, by $(\mathrm{A}-1)$, we have the diagram

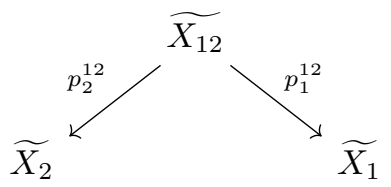


in $\widetilde{\mathcal{C}}$ such that $p_{2}^{12}$ has a section $d_{12}^{2}$ with $p_{1}^{12} d_{12}^{2}=\widetilde{f}_{1}$. Then put $\alpha_{\widetilde{f}_{1}}$ as the invertible 2-morphism

$$
{\widetilde{f_{1}}}^{*} \stackrel{\sim}{\longrightarrow} d_{12}^{2 *} p_{1}^{12 *} \stackrel{\sim}{\longrightarrow} d_{12}^{2 *} p_{2}^{12 *}\left(p_{2}^{12 *}\right)^{-1} p_{1}^{12 *} \stackrel{\sim}{\longrightarrow}\left(p_{2}^{12 *}\right)^{-1} p_{1}^{12 *}=H\left(f_{1}\right)
$$

in $\mathcal{D}$ where $f_{1}=F\left(\widetilde{f}_{1}\right)$.

Now, we will verify the axioms of pseudonatural transformations for $\alpha$.

(1) Let $\widetilde{X_{2}} \stackrel{\widetilde{f_{1}}}{\rightarrow} \widetilde{X_{1}}$ be a morphism in $\widetilde{C}$, and put $f_{1}=F\left(\widetilde{f_{1}}\right)$. When $\widetilde{f}_{1}$ is the identity morphism, we have to show that the diagram

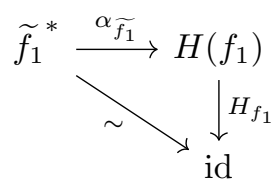

in $\mathcal{D}$ commutes. It follows from construction.

(2) Let $\widetilde{X_{3}} \stackrel{\widetilde{f_{2}}}{\rightarrow} \widetilde{X_{2}} \stackrel{\widetilde{f_{1}}}{\rightarrow} \widetilde{X_{1}}$ be morphisms in $\widetilde{C}$, and put

$$
f_{1}=F\left(\widetilde{f}_{1}\right), \quad f_{2}=F\left(\widetilde{f}_{2}\right) .
$$

We have to show that the diagram

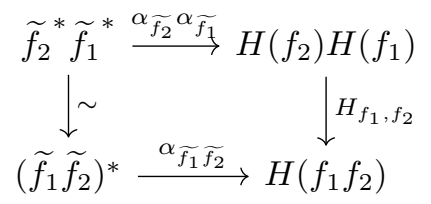

in $\mathcal{D}$ commutes. Since $p_{2}^{12 *}, p_{3}^{23 *}, p_{13}^{123 *}$, and $p_{23}^{123 *}$ are invertible, we have isomorphisms

$$
\begin{array}{cc}
d_{12}^{2 *} \stackrel{\sim}{\longrightarrow}\left(p_{2}^{12 *}\right)^{-1}, & d_{23}^{3 *} \stackrel{\sim}{\longrightarrow}\left(p_{3}^{23 *}\right)^{-1}, \\
d_{123}^{13 *} \stackrel{\sim}{\longrightarrow}\left(p_{13}^{123 *}\right)^{-1}, & d_{123}^{23 *} \stackrel{\sim}{\longrightarrow}\left(p_{23}^{123 *}\right)^{-1} .
\end{array}
$$

Then we have the diagram

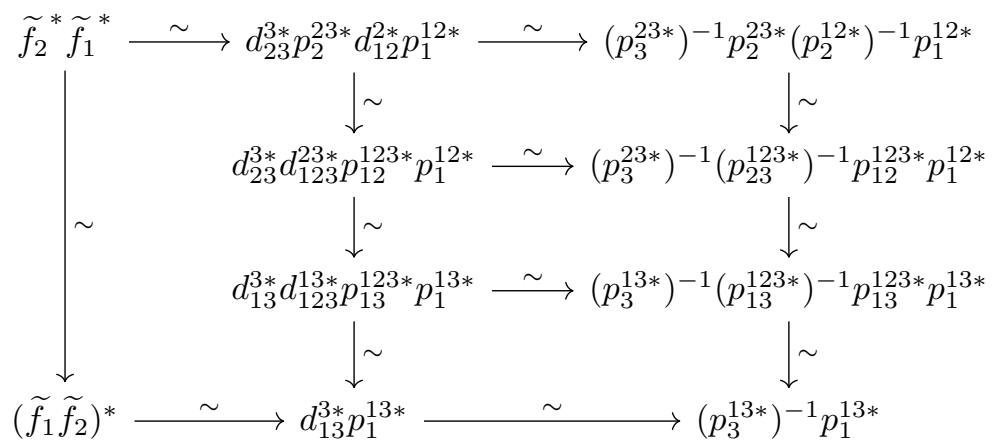

in $\mathcal{D}$, and its big outside diagram is (3.6.1). Since each small squares of the above diagram commute, (3.6.1) commutes. 
We have verified the axioms of pseudonatural transformations for $\alpha$, so $\alpha$ is a pseudonatural transformation. Since $\alpha\left(\widetilde{X_{1}}\right)$ is an equivalence for any object $\widetilde{X_{1}}$ of $\widetilde{C}, \alpha$ is a pseudonatural equivalence. Thus we have proven the following theorem.

Theorem 3.7. Assume the conditions from (A-1) to (A-3), and assume that $g^{*}$ is an equivalence for any $g \in \mathscr{W}$. Then there is a pseudofunctor $H: \mathcal{C} \rightarrow \mathcal{D}$ and a pseudonatural equivalence $\alpha: G \rightarrow H \circ F$ making the diagram

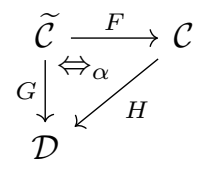

commutes.

3.8. Now, we will prove that the above construction is functorial in the following sense.

Theorem 3.9. Assume the conditions from $(\mathrm{A}-1)$ to $(\mathrm{A}-3)$, and assume that $g^{*}$ is an equivalence for any $g \in \mathscr{W}$. Let

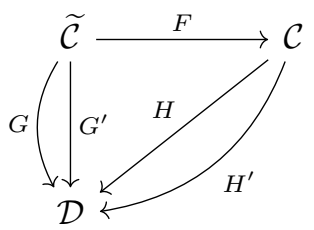

be a diagram in $\mathcal{C}$ extending (3.1.1) where $G^{\prime}$ and $H^{\prime}$ are contravariant pseudofunctors such that $G^{\prime}(g)$ is invertible for any morphism $g \in \mathscr{W}$, and let

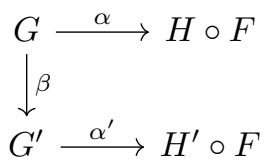

be a diagram of pseudofunctors (i.e., $\alpha, \beta$, and $\alpha^{\prime}$ are pseudonatural transformations) such that $\alpha$ and $\alpha^{\prime}$ are pseudonatural equivalences. Then there is a unique pseudonatural transformation $\gamma: H \rightarrow H^{\prime}$ up to isomorphisms such that the induced pseudonatural transformation

$$
H \circ F \rightarrow H^{\prime} \circ F
$$

makes the above diagram commutative.

Proof. We may assume that $\alpha$ and $\alpha^{\prime}$ are the identity 2-morphisms for simplicity. We will first construct $\gamma$. For any object $X_{1}$ of $\mathcal{C}$, put $\gamma\left(X_{1}\right)$ as the 1-morphism $H\left(X_{1}\right) \rightarrow H^{\prime}\left(X_{1}\right)$ given by

$$
H\left(X_{1}\right)=G\left(\widetilde{X_{1}}\right) \stackrel{\beta\left(\widetilde{X_{1}}\right)}{\longrightarrow} G^{\prime}\left(\widetilde{X_{1}}\right)=H^{\prime}\left(X_{1}\right) .
$$

For any morphism $X_{2} \stackrel{f_{1}}{\rightarrow} X_{1}$ in $\mathcal{C}$, consider the diagram

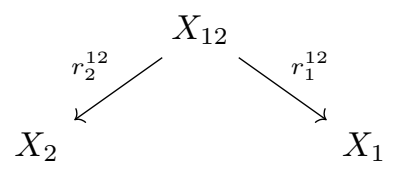


in $\mathcal{C}$ that is the image of the diagram in $(\mathrm{A}-1)$ via $F$. Put

$$
H_{1}\left(f_{1}\right)=H\left(r_{2}^{12}\right)^{-1} H\left(r_{1}^{12}\right), \quad H_{1}^{\prime}\left(f_{1}\right)=H^{\prime}\left(r_{2}^{12}\right)^{-1} H^{\prime}\left(r_{1}^{12}\right) .
$$

Then we have the invertible 2-morphism

$$
\mu_{f_{1}}: H_{1}\left(f_{1}\right) \rightarrow H\left(f_{1}\right)
$$

in $\mathcal{D}$ given by the composition

$$
H\left(r_{2}^{12}\right)^{-1} H\left(r_{1}^{12}\right) \stackrel{\sim}{\longrightarrow} H\left(\left(r_{2}^{12}\right)^{-1}\right) H\left(r_{1}^{12}\right) \stackrel{H_{r_{1}^{12}} \stackrel{\left(r_{12}^{2}\right)^{-1}}{\longrightarrow}}{\longrightarrow} H\left(r_{1}^{12}\left(r_{2}^{12}\right)^{-1}\right) \stackrel{\sim}{\longrightarrow} H\left(f_{1}\right) .
$$

We similarly have the invertible 2-morphism

$$
\mu_{f_{1}}^{\prime}: H_{1}^{\prime}\left(f_{1}\right) \rightarrow H^{\prime}\left(f_{1}\right)
$$

in $\mathcal{D}$. We also have the invertible 2-morphism

$$
\eta_{f_{1}}: \gamma\left(X_{2}\right) H_{1}\left(f_{1}\right) \longrightarrow H_{1}^{\prime}\left(f_{1}\right) \gamma\left(X_{1}\right)
$$

in $\mathcal{D}$ given by the composition

$$
\begin{aligned}
\gamma\left(X_{2}\right) H\left(r_{2}^{12}\right)^{-1} H\left(r_{1}^{12}\right) & =\beta\left(\widetilde{X_{2}}\right) G\left(p_{2}^{12}\right)^{-1} G\left(p_{1}^{12}\right) \rightarrow G^{\prime}\left(p_{2}^{12}\right)^{-1} \beta\left(\widetilde{X_{12}}\right) G\left(p_{1}^{12}\right) \\
& \stackrel{\beta_{p_{12}^{12}}}{\rightarrow} G^{\prime}\left(p_{2}^{12}\right)^{-1} G\left(p_{1}^{12}\right) \beta\left(\widetilde{X_{1}}\right)=H_{1}^{\prime}\left(r_{2}^{12}\right)^{-1} H_{1}^{\prime}\left(r_{1}^{12}\right) \gamma\left(X_{1}\right) .
\end{aligned}
$$

Here, the second arrow is obtained by applying $H^{\prime}\left(r_{2}^{12}\right)^{-1}$ to the left side and $H\left(r_{1}^{12}\right)^{-1}$ to the right side of the 2-morphism

$$
\beta_{p_{2}^{12}}^{-1}: H^{\prime}\left(r_{2}^{12}\right) \beta\left(\widetilde{X_{2}}\right) \longrightarrow \beta\left(\widetilde{X_{12}}\right) H\left(r_{2}^{12}\right)
$$

in $\mathcal{D}$. Then put $\gamma_{f_{1}}$ as the 2-morphism

$$
\gamma\left(X_{2}\right) H\left(f_{1}\right) \rightarrow H^{\prime}\left(f_{2}\right) \gamma\left(X_{1}\right)
$$

in $\mathcal{D}$ given by the composition

$$
\gamma\left(X_{2}\right) H\left(f_{1}\right) \stackrel{\mu_{f_{1}}^{-1}}{\longrightarrow} \gamma\left(X_{2}\right) H_{1}\left(f_{1}\right) \stackrel{\eta_{f_{1}}}{\longrightarrow} H_{1}^{\prime}\left(f_{1}\right) \gamma\left(X_{1}\right) \stackrel{\mu_{f_{1}}^{\prime}}{\longrightarrow} H^{\prime}\left(f_{1}\right) \gamma\left(X_{1}\right) .
$$

Now, we will verify the axioms of pseudonatural transformations for $\gamma$.

(1) For any morphism $X_{2} \stackrel{f_{1}}{\rightarrow} X_{1}$, when $X_{1}=X_{2}$ and $f_{1}$ is the identity morphism, we have to show that the diagram

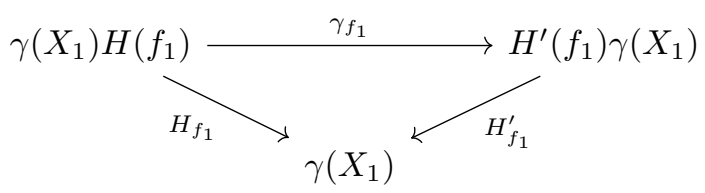


in $\mathcal{D}$ commutes. By $(\mathrm{A}-1), p_{2}^{12}$ has a section $d_{12}^{2}$ such that $\widetilde{f}_{1}=p_{1}^{12} d_{12}^{2}$, and the above diagram is the big outside diagram of the diagram

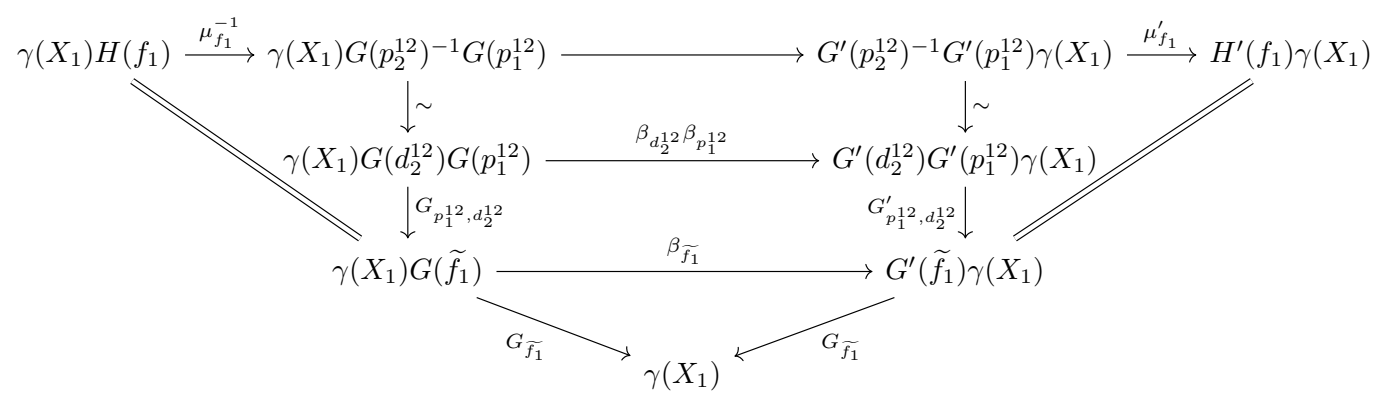

in $\mathcal{D}$, whose small diagrams commute. Here, the not denoted arrow in the first row is the 2-morphism induced by $\gamma$. Thus (3.9.1) commutes.

(2) For any morphisms $X_{3} \stackrel{f_{2}}{\rightarrow} X_{2} \stackrel{f_{1}}{\rightarrow} X_{1}$ in $\mathcal{C}$, consider the commutative diagram

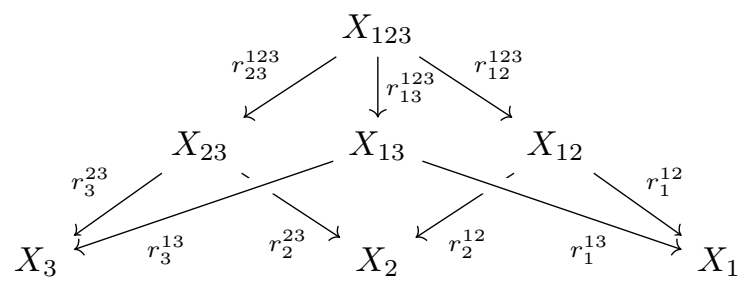

in $\mathcal{C}$ that is the image of the diagram in $(\mathrm{A}-2)$ via $F: \widetilde{\mathcal{C}} \rightarrow \mathcal{C}$. Put

$$
\begin{aligned}
& U\left(f_{2}\right)=H\left(r_{2}^{12}\right)^{-1} H\left(r_{1}^{12}\right), \quad U\left(f_{1} f_{2}\right)=H\left(r_{3}^{13}\right)^{-1} H\left(r_{1}^{13}\right), \\
& U^{\prime}\left(f_{2}\right)=H^{\prime}\left(r_{2}^{12}\right)^{-1} H^{\prime}\left(r_{1}^{12}\right), \quad U^{\prime}\left(f_{1} f_{2}\right)=H^{\prime}\left(r_{3}^{13}\right)^{-1} H^{\prime}\left(r_{1}^{13}\right),
\end{aligned}
$$

and consider the 2-morphisms

$$
U\left(f_{2}\right) U\left(f_{1}\right) \stackrel{U_{f_{1}, f_{2}}}{\longrightarrow} U\left(f_{1} f_{2}\right), \quad U^{\prime}\left(f_{2}\right) U\left(f_{1}\right) \stackrel{U_{f_{1}, f_{2}}^{\prime}}{\longrightarrow} U^{\prime}\left(f_{1} f_{2}\right)
$$

in $\mathcal{D}$ defined as in $(\underline{3.3}(4))$. We also have the 2-morphisms

$$
\begin{gathered}
U\left(f_{2}\right) \stackrel{\mu_{f_{2}}}{\longrightarrow} H\left(f_{2}\right), \quad U\left(f_{1} f_{2}\right) \stackrel{\mu_{f_{1} f_{2}}}{\longrightarrow} H\left(f_{1} f_{2}\right), \\
U^{\prime}\left(f_{2}\right) \stackrel{\mu_{f_{2}}^{\prime}}{\longrightarrow} H^{\prime}\left(f_{2}\right), \quad U^{\prime}\left(f_{1} f_{2}\right) \stackrel{\mu_{f_{1} f_{2}}^{\prime}}{\longrightarrow} H^{\prime}\left(f_{1} f_{2}\right), \\
\gamma\left(X_{3}\right) U\left(f_{2}\right) \stackrel{\eta_{f_{2}}}{\longrightarrow} U^{\prime}\left(f_{2}\right) \gamma\left(X_{2}\right), \quad \gamma\left(X_{3}\right) U\left(f_{1} f_{2}\right) \stackrel{\eta_{f_{1} f_{2}}}{\longrightarrow} U^{\prime}\left(f_{1} f_{2}\right) \gamma\left(X_{1}\right)
\end{gathered}
$$


in $\mathcal{D}$ defined as $\mu\left(f_{1}\right), \mu^{\prime}\left(f_{1}\right)$, and $\eta\left(f_{1}\right)$. Then we have the diagram

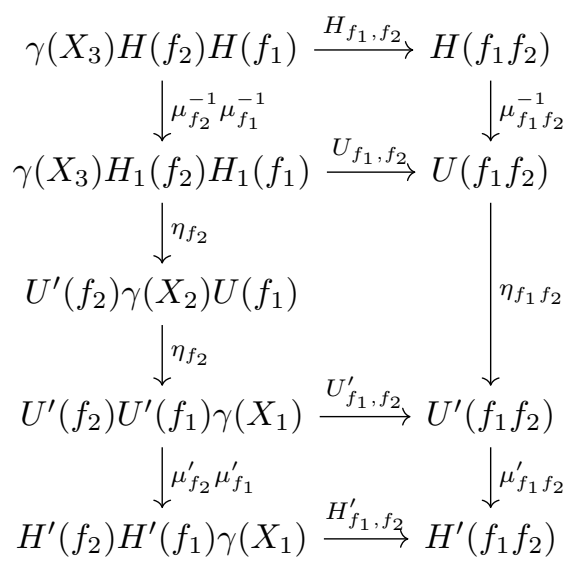

in $\mathcal{D}$. The middle diagram commutes as in (3.4) since $\beta: G \rightarrow G^{\prime}$ is a pseudonatural transformation. The top and bottom diagrams commute as in the proof that (3.6.1) commutes. Thus the above diagram commutes, so we obtain the commutative diagram

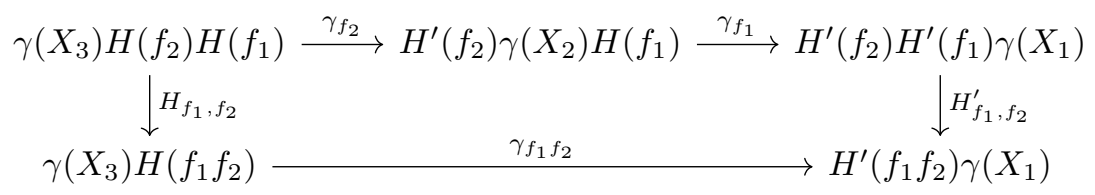

in $\mathcal{D}$.

We have verified the axioms of pseudonatural transformations for $\gamma$, so $\gamma$ is a pseudonatural transformation. The commutativity of the diagram

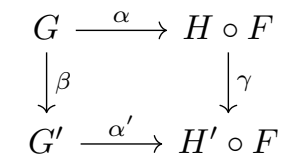

of pseudofunctors follows from construction. Thus the remaining part is the uniqueness of $\gamma$ up to isomorphisms. If $\gamma^{\prime}: H \rightarrow H^{\prime}$ is another pseudonatural transformation such that $\alpha^{\prime} \beta$ is isomorphic to $\gamma^{\prime} \alpha$, then $\gamma \alpha$ and $\gamma^{\prime} \alpha$ are isomorphic. From (2.4), the data of the isomorphism is a collection of invertible 2-morphisms

$$
\Theta_{\widetilde{Y_{1}}}: \gamma \alpha\left(\widetilde{Y_{1}}\right) \rightarrow \gamma^{\prime} \alpha\left(\widetilde{Y_{1}}\right)
$$

in $\mathcal{D}$ for any object $\widetilde{Y_{1}}$ of $\widetilde{\mathcal{C}}$ such that for any morphism $\widetilde{g_{1}}: \widetilde{Y_{2}} \rightarrow \widetilde{Y_{1}}$ in $\widetilde{\mathcal{C}}$, the diagram

$$
\begin{gathered}
\gamma \alpha\left(\widetilde{Y_{1}}\right) \stackrel{\Theta_{\widetilde{Y_{1}}}}{\longrightarrow} \gamma^{\prime} \alpha\left(\widetilde{Y_{1}}\right) \\
\quad \downarrow(\gamma \alpha)_{\widetilde{g_{1}}} \quad \downarrow\left(\gamma^{\prime} \alpha\right)_{\widetilde{g_{1}}} \\
\gamma \alpha\left(\widetilde{Y_{2}}\right) \stackrel{\Theta_{\widetilde{Y_{2}}}}{\longrightarrow} \gamma^{\prime} \alpha\left(\widetilde{Y_{2}}\right)
\end{gathered}
$$


in $\mathcal{D}$ commutes. Then for any object $X_{1}$ of $\mathcal{C}$, the data gives the collection of invertible 2-morphisms

$$
\Phi_{X_{1}}: \gamma\left(X_{1}\right) \rightarrow \gamma^{\prime}\left(X_{1}\right)
$$

in $\mathcal{D}$. The condition that $\Gamma$ is a modification is the commutativity of the diagram

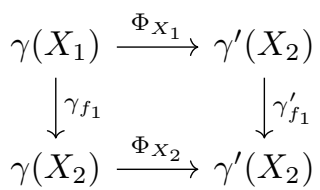

in $\mathcal{D}$ for any morphism $f_{1}: X_{2} \rightarrow X_{1}$ in $\mathcal{C}$. It follows from showing that the diagram

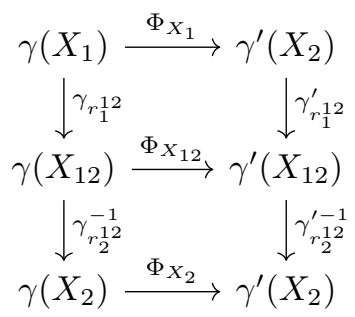

in $\mathcal{D}$ commutes, which is true because of the commutativity of (3.9.2). Thus $\Phi: \gamma \rightarrow \gamma^{\prime}$ is a modification, which is an isomorphism since $\Phi_{X_{1}}$ is invertible for any object $X$ of $\mathcal{C}$.

\section{Categories with immersions}

Definition 4.1. A category with immersions is the data of $(\mathcal{C}, c: \mathcal{I} \rightarrow \mathcal{J})$ with the following axioms.

$(\mathrm{D}-1) \mathcal{C}$ is a category, and $\mathcal{I}$ and $\mathcal{J}$ are classes of monomorphisms in $\mathcal{C}$, and $c: \mathcal{I} \rightarrow \mathcal{J}$ is a function. Assume that $\mathcal{C}$ has fiber products, an initial object, and a final object. A morphism in $\mathcal{I}$ is called a closed immersions, and a morphism in $\mathcal{J}$ is called an open immersions. When $i \in \mathcal{I}$, $c(i)$ is called the complement of $i$.

(D-2) For any closed immersion $Z \rightarrow S$ in $\mathcal{C}$, the morphism $c(Z \rightarrow S)$ is an open immersion whose target is $S$.

(D-3) Isomorphisms are closed immersions and open immersions, and closed immersions and open immersions are stable by compositions and pullbacks.

(D-4) For any commutative diagram

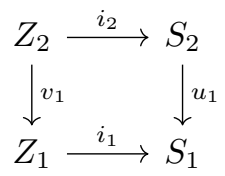

in $\mathcal{C}$ where $i_{1}$ and $i_{2}$ are closed immersions with complements $j_{1}: U_{1} \rightarrow S_{1}$ and $j_{2}: U_{2} \rightarrow S_{2}$ respectively, the projection $U_{1} \times_{S_{1}} S_{2} \rightarrow S_{2}$ has a factorization

$$
U_{1} \times_{S_{1}} S_{2} \stackrel{j_{2}^{\prime}}{\rightarrow} U_{2} \stackrel{j_{2}}{\rightarrow} S_{2}
$$


for some open immersion $j_{2}^{\prime}$. Moreover, $j_{2}^{\prime}$ is an isomorphism when the diagram (4.1.1) is Cartesian.

If no confusion seems likely to arise, we simply write $\mathcal{C}$ for $(\mathcal{C}, c: \mathcal{I} \rightarrow \mathcal{J})$.

Definition 4.2. Let $\mathcal{C}$ be a category with immersions, and let $S$ be an object of $\mathcal{C}$. We say that $S$ is separated if the diagonal morphism $S \rightarrow S \times S$ is a closed immersion.

Proposition 4.3. Let $\mathcal{C}$ be a category with immersions, and let $i_{1}: Z \rightarrow S_{1}$ and $i_{2}: Z \rightarrow S_{2}$ be closed immersions in $\mathcal{C}$. If $Z$ is separated, then the morphism

$$
i: Z \rightarrow S_{1} \times S_{2}
$$

induced by $i_{1}$ and $i_{2}$ is a closed immersion.

Proof. The morphism $i$ has the factorization

$$
Z \stackrel{\Delta}{\longrightarrow} Z \times Z \stackrel{\text { id } \times i_{2}}{\longrightarrow} Z \times S_{2} \stackrel{i_{1} \times \mathrm{id}}{\longrightarrow} S_{1} \times S_{2}
$$

where $\Delta$ denotes the diagonal morphism. The first arrow is a closed immersion since $Z$ is separated, and the second and third arrows are closed immersions by (D-3). Thus the composition is also a closed immersion by (D-3).

Example 4.4. Let $\mathcal{C}$ be a category with immersions, and let $\mathcal{E}$ be a full subcategory of $\mathcal{C}$ such that

(i) for any object $Z_{1}$ of $\mathcal{C}$, there is a closed immersion $Z_{1} \rightarrow S_{1}$ with $S_{1} \in$ ob $\mathcal{E}$,

(ii) for any open immersion $U_{1} \rightarrow S_{1}$ in $\mathcal{C}$, if $S_{1}$ is in $\mathcal{E}$, then $U_{1}$ is also in $\mathcal{E}$.

We will construct some categories with immersions and functors that will be used frequently later.

The category $\mathcal{E}$ has a structure of immersions as follows. A morphism $S_{2} \rightarrow S_{1}$ in $\mathcal{E}$ is a closed immersion if its image in $\mathcal{C}$ is a closed immersion. A morphism $S_{2} \rightarrow S_{1}$ in $\mathcal{E}$ is an open immersion if its image in $\mathcal{C}$ is an open immersion. The complement of a closed immersion is defined from the complement function in $\mathcal{C}$, which is possible because of (ii). The axioms (D-3) and (D-4) for $\mathcal{E}$ follows from those for $\mathcal{C}$.

Consider the category $\widetilde{\mathcal{C}}$ where objects are morphisms $\left(Z_{1} \rightarrow S_{1}\right)$ in $\mathcal{C}$ and morphisms are commutative diagrams

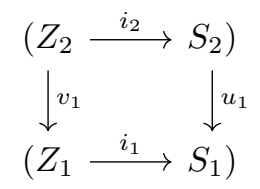

in $\mathcal{C}$. Then $\widetilde{\mathcal{C}}$ has a structure of immersions as follows. A closed immersion in $\widetilde{\mathcal{C}}$ is a morphism that is isomorphic to a commutative diagram

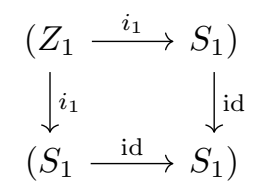


in $\mathcal{C}$ where $i_{1}$ is a closed immersion in $\mathcal{C}$. An open immersion in $\widetilde{\mathcal{C}}$ is a morphism that is isomorphic to a commutative diagram

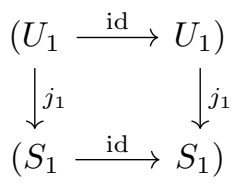

in $\mathcal{C}$ where $j_{1}$ is an open immersion in $\mathcal{C}$. The complement of a closed immersion in $\widetilde{\mathcal{C}}$ given by (4.4.1) is the open immersion in $\widetilde{\mathcal{C}}$ given by (4.4.2) if $j_{1}$ is the complement of $i_{1}$. The axioms (D-3) and (D-4) for $\widetilde{\mathcal{C}}$ follows from those for $\mathcal{C}$.

Let $S_{1}$ be an object of $\mathcal{C}$. We often write $S_{1}$ for the object $\left(S_{1} \rightarrow S_{1}\right)$ in $\widetilde{\mathcal{C}}$ when no confusion seems likely to arise. Let $S_{2} \rightarrow S_{1}$ be a morphism in $\mathcal{C}$. We often write $S_{2} \rightarrow S_{1}$ for the morphism in $\widetilde{\mathcal{C}}$ given by the commutative diagram

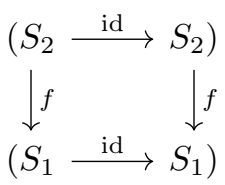

in $\mathcal{C}$ when no confusion seems likely to arise.

Let $U: \mathcal{E} \rightarrow \widetilde{\mathcal{C}}$ denote the functor mapping object $S_{1}$ to $S_{1}$ and mapping morphism $S_{2} \rightarrow S_{1}$ to $S_{2} \rightarrow S_{1}$. Then let $F: \widetilde{\mathcal{C}} \rightarrow \mathcal{C}$ denote the functor mapping object $\left(Z_{1} \rightarrow S_{1}\right)$ to $Z_{1}$ and mapping morphism given by the diagram

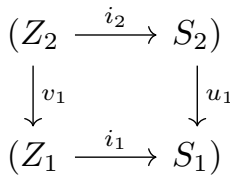

in $\mathcal{C}$ to $v_{1}: Z_{2} \rightarrow Z_{1}$.

Let $\mathscr{P}$ be a class of morphisms of $\mathcal{C}$ containing all isomorphisms and stable by compositions and pullbacks. Then we denote by $\widetilde{\mathscr{P}}$ the class of morphisms in $\widetilde{\mathcal{C}}$ given by a Cartesian diagram

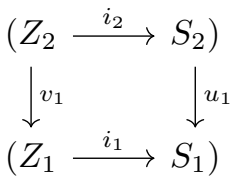

such that $u_{1}$ is in $\mathscr{P}$. We also put $\mathscr{P}^{\prime}=W^{-1}(\mathscr{P})$.

Remark 4.5. In (4.4), we are particularly interested in the case is when $\mathcal{C}=\mathscr{S}, \mathcal{E}=\mathscr{S}^{s m}$, and $\mathscr{P}=S m$.

4.6. Under the notations and hypothesis of (4.4), we will show the conditions from (A-1) to (A-3) so that we can use (3.7) and (3.9). Consider the category $\mathcal{C}^{\prime}$ where objects are morphisms $\left(Z_{1} \rightarrow S_{1}\right)$ 
in $\mathcal{C}$ and morphisms are commutative diagrams

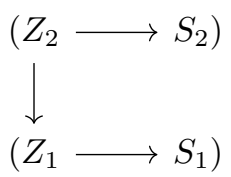

in $\mathcal{C}$. Then $\mathcal{C}^{\prime}$ is equivalent to $\mathcal{C}$, and we have the obvious functor

$$
F^{\prime}: \widetilde{\mathcal{C}} \rightarrow \mathcal{C}^{\prime}
$$

We will verify the conditions from (A-1) to $(\mathrm{A}-3)$ for $F^{\prime}$.

Let $X_{4} \stackrel{f_{3}}{\rightarrow} X_{3} \stackrel{f_{2}}{\rightarrow} X_{2} \stackrel{f_{1}}{\rightarrow} X_{1}$ be morphisms in $\mathcal{C}$ given by the diagram

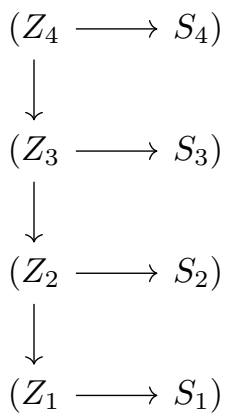

in $\mathcal{C}$. Then put

$$
\begin{aligned}
& \widetilde{X_{1}}=X_{1}, \quad \widetilde{X_{2}}=X_{2}, \quad \widetilde{X_{3}}=X_{3}, \quad \widetilde{X_{4}}=X_{4}, \\
& \widetilde{X_{12}}=\left(Z_{2} \rightarrow S_{1} \times S_{2}\right), \quad \widetilde{X_{13}}=\left(Z_{3} \rightarrow S_{1} \times S_{3}\right), \quad \widetilde{X_{14}}=\left(Z_{4} \rightarrow X_{1} \times X_{4}\right) \text {, } \\
& \widetilde{X_{23}}=\left(Z_{3} \rightarrow S_{2} \times S_{3}\right), \quad \widetilde{X_{24}}=\left(Z_{4} \rightarrow S_{2} \times S_{4}\right), \quad \widetilde{X_{34}}=\left(Z_{4} \rightarrow X_{3} \times X_{4}\right) \text {, } \\
& \widetilde{X_{123}}=\left(Z_{3} \rightarrow S_{1} \times S_{2} \times S_{3}\right), \quad \widetilde{X_{124}}=\left(Z_{4} \rightarrow S_{1} \times S_{2} \times S_{4}\right), \\
& \widetilde{X_{134}}=\left(Z_{4} \rightarrow S_{1} \times S_{3} \times S_{4}\right), \quad \widetilde{X_{234}}=\left(Z_{4} \rightarrow S_{2} \times S_{3} \times S_{4}\right), \\
& \widetilde{X_{1234}}=\left(Z_{4} \rightarrow S_{1} \times S_{2} \times S_{3} \times S_{4}\right)
\end{aligned}
$$

where the morphisms are induced by compositions of morphisms in (4.6.1). By (4.3), these are in $\widetilde{\mathcal{C}}$. We also have the morphisms

$$
\begin{aligned}
p_{1}^{12}: \widetilde{X_{12}} & \rightarrow \widetilde{X_{1}}, \quad \ldots, \quad p_{234}^{1234}: \widetilde{X_{1234}} \rightarrow \widetilde{X_{234}}, \\
d_{12}^{2}: \widetilde{X_{2}} & \rightarrow \widetilde{X_{12}}, \quad \ldots, \quad d_{123}^{23}: \widetilde{X_{23}} \rightarrow \widetilde{X_{123}}
\end{aligned}
$$

induced by the projections

$$
S_{1} \times S_{2} \rightarrow S_{1}, \quad \ldots, \quad S_{1} \times S_{2} \times S_{3} \times S_{4} \rightarrow S_{2} \times S_{3} \times S_{4}
$$

and the graph morphisms

$$
S_{2} \times S_{1} \times S_{2}, \quad \ldots, \quad S_{2} \times S_{3} \rightarrow S_{1} \times S_{2} \times S_{3}
$$

respectively. Our construction of $\widetilde{X_{1}}, \ldots, \widetilde{X_{1234}}, p_{1}^{12}, \ldots, p_{234}^{1234}$, and $d_{12}^{2}, \ldots, d_{123}^{23}$ satisfy the conditions from $(\mathrm{A}-1)$ to $(\mathrm{A}-3)$ for $F^{\prime}$. 
Definition 4.7. Let $\mathcal{C}$ be a category with immersions, let $\mathscr{P}$ be a class of morphisms in $\mathcal{C}$ containing all isomorphisms and stable by compositions and pullbacks, let $H: C \rightarrow \operatorname{Tri}^{\otimes}$ be a $\mathscr{P}$-premotivic pseudofunctor, let $i$ be a closed immersion in $\mathcal{C}$, and let $j$ denote the complement of $i$. We say that $H$ satisfies $\left(\operatorname{Loc}_{i}\right)$ if $i_{*}$ is fully faithful and the pair of functors $\left(i^{*}, j^{*}\right)$ is conservative.

We also say that $H$ satisfies the localization property, denoted by (Loc), if

(1) For any Cartesian diagram

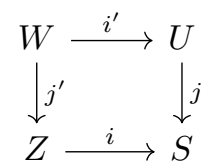

where $i$ is a closed immersion with complement $j$, we have $H(W)=0$,

(2) $H$ satisfies $\left(\operatorname{Loc}_{i}\right)$ for any closed immersion $i$ in $\mathcal{C}$.

4.8. Under the notations and hypotheses of (4.7), let $i: Z \rightarrow S$ be a closed immersion in $\mathcal{C}$, and let $j$ denote its complement. We list several consequences of (Loc) and the axioms from (B-1) to (B-4) whose proofs are the same as those in [CD12, §2.3]

(1) The functor $i_{*}$ admits a right adjoint $i$.

(2) There exists a unique natural transformation $\partial_{i}: i_{*} i^{*} \rightarrow j_{\sharp} j^{*}[1]$ such that the triangle

$$
j_{\sharp} j^{*} \stackrel{a d^{\prime}}{\longrightarrow} \text { id } \stackrel{a d}{\longrightarrow} i_{*} i^{*} \stackrel{\partial_{i}}{\longrightarrow} j_{\sharp} j^{*}[1]
$$

is distinguished.

(3) There exists a unique natural transformation $\partial_{i}: j_{*} j^{*} \rightarrow i_{*} i^{i}[1]$ such that the triangle

$$
i_{*} i^{!} \stackrel{a d^{\prime}}{\longrightarrow} \mathrm{id} \stackrel{a d}{\longrightarrow} j_{*} j^{*} \stackrel{\partial_{i}}{\longrightarrow} i_{*} i^{!}[1]
$$

is distinguished.

(4) We have $i^{*} j_{\sharp}=0$.

(5) The unit id $\stackrel{a d}{\longrightarrow} j^{*} j_{\sharp}$ is an isomorphism.

4.9. Under the notations and hypotheses of (4.7), assume $\mathcal{C}=\mathscr{S}$. A consequence of (Loc) in CD12, 3.3.4] is as follows. Consider a Cartesian diagram

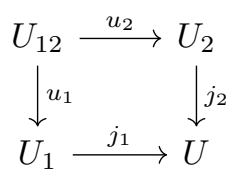

in $\mathscr{S}$ where $j_{1}$ and $j_{2}$ are open immersions, and put $j_{12}=j_{1} u_{1}$. Then there is a distinguished triangle

$$
j_{12 \sharp j_{12}} \longrightarrow j_{1 \sharp} j_{1}^{*} \oplus j_{2 \sharp} j_{2}^{*} \stackrel{a d^{\prime}+a d^{\prime}}{\longrightarrow} \text { id } \longrightarrow j_{12 \sharp} j_{12}^{*}[1]
$$


where the first arrow is induced by the natural transformations

$$
\begin{aligned}
& j_{12 \sharp} j_{12}^{*} \stackrel{\sim}{\longrightarrow} j_{1 \sharp} u_{1 \sharp} u_{1}^{*} j_{1}^{*} \stackrel{a d^{\prime}}{\longrightarrow} j_{1 \sharp} j_{1}^{*}, \\
& j_{12 \sharp j_{12}} \stackrel{\sim}{\longrightarrow} j_{2 \sharp} u_{2 \sharp} u_{2}^{*} j_{2}^{*} \stackrel{-a d^{\prime}}{\longrightarrow} j_{2 \sharp} j_{2}^{*} .
\end{aligned}
$$

The distinguished triangled is called the Mayer-Vietoris distinguished triangle. Its right adjoint

$$
\mathrm{id} \longrightarrow j_{1 *} j_{1}^{*} \oplus j_{2 *} j_{2}^{*} \longrightarrow j_{12 *} j_{12}^{*} \longrightarrow \mathrm{id}[1]
$$

is also a distinguished triangle.

Proposition 4.10. Under the notations and hypotheses of (4.7), consider a Cartesian diagram

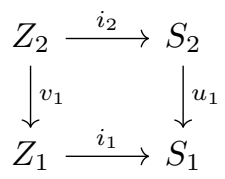

in $\mathcal{C}$ where $i_{1}$ is a closed immersion. Assume that $H$ satisfies (Loc). Then the exchange transformation

$$
u_{1}^{*} i_{1 *} \stackrel{E x}{\longrightarrow} i_{2 *} v_{1}^{*}
$$

given by the composition

$$
u_{1}^{*} i_{1 *} \stackrel{a d}{\longrightarrow} u_{1}^{*} i_{1 *} v_{1 *} v_{1}^{*} \stackrel{\sim}{\longrightarrow} u_{1}^{*} u_{1 *} i_{2 *} v_{1}^{*} \stackrel{a d^{\prime}}{\longrightarrow} i_{2 *} v_{1}^{*}
$$

is an isomorphism.

Proof. Consider the diagram

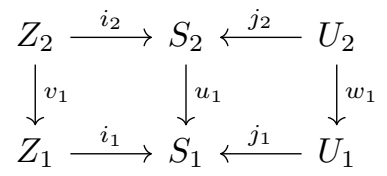

in $\mathcal{C}$ where each square is Cartesian and $j_{1}$ denotes the complement of $i_{1}$. By (D-4), $j_{2}$ is the complement of $i_{2}$. To show the statement, by (Loc), it suffices to show that the natural transformations

$$
j_{2}^{*} u_{1}^{*} i_{1 *} \stackrel{E x}{\longrightarrow} j_{2}^{*} i_{2 *} v_{1}^{*}, \quad i_{2}^{*} u_{1}^{*} i_{1 *} \stackrel{E x}{\longrightarrow} i_{2}^{*} i_{2 *} v_{1}^{*}
$$

are isomorphisms. The first arrow is an isomorphism since $j_{2}^{*} i_{2 *}=0$ and $j_{2}^{*} u_{1}^{*} i_{1 *} \cong w_{1}^{*} j_{1}^{*} u_{1 *}=0$. Hence the remaining is to show that the second arrow is an isomorphism.

Consider the commutative diagram

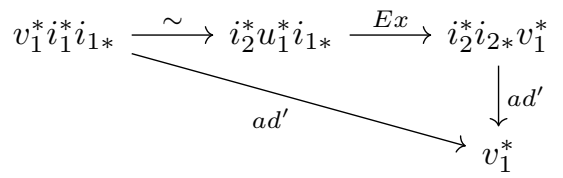

of functors. The vertical arrow and diagonal arrow are isomorphisms by (Loc), so the right horizontal arrow is an isomorphism. Thus the second arrow of (4.10.1) is an isomorphism. 
Proposition 4.11. Under the notations and hypotheses of (4.10), assume that $u_{1}$ is in $\mathscr{P}$. Then the exchange transformation

$$
u_{1 \sharp} i_{2 *} \stackrel{E x}{\longrightarrow} i_{1 *} v_{1 \sharp}
$$

given by the composition

$$
u_{1 \sharp} i_{2 *} \stackrel{a d}{\longrightarrow} i_{1 *} i_{1}^{*} u_{1 \sharp} i_{2 *} \stackrel{E x^{-1}}{\longrightarrow} i_{1 *} v_{1 \sharp} i_{2}^{*} i_{2 *} \stackrel{a d^{\prime}}{\longrightarrow} i_{1 *} v_{1 \sharp}
$$

is an isomorphism.

Proof. Note first that the second arrow in the composition is defined and an isomorphism by (4.10). The third arrow is an isomorphism by (Loc). Thus the remaining is to show that the first arrow is an isomorphism.

Consider the Cartesian diagram

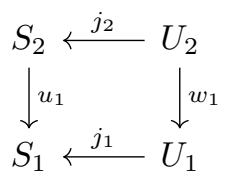

in $\mathcal{C}$ where $j_{1}$ denotes the complement of $i_{1}$. Then by (D-4), $j_{2}$ is the complement of $i_{2}$. By (Loc), it suffices to show that the natural transformations

$$
\begin{aligned}
& j_{1}^{*} u_{1 \sharp} i_{2 *} \stackrel{E x}{\longrightarrow} j_{1}^{*} i_{1 *} v_{1 \sharp}, \\
& i_{1}^{*} u_{1 \sharp} i_{2 *} \stackrel{E x}{\longrightarrow} i_{1}^{*} i_{1 *} v_{1 \sharp}
\end{aligned}
$$

are isomorphisms. By (B-3) and $(4.8(4))$, we have

$$
j_{1}^{*} u_{1 \sharp} i_{2 *} \cong w_{1 \sharp} j_{2}^{*} i_{2 *}=0, \quad j_{1}^{*} i_{1 *} v_{1 \sharp}=0,
$$

so (4.11.1) is an isomorphism. Thus the remaining is to show that (4.11.2) is an isomorphism. Consider the commutative diagram

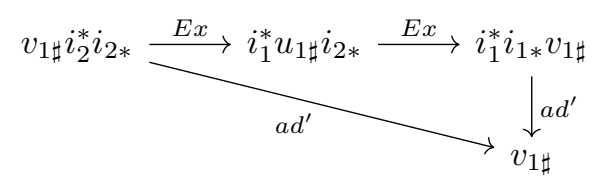

of functors. The vertical arrow and diagonal arrow are isomorphisms by (Loc), and the left horizontal arrow is an isomorphism by (4.10). Thus the right horizontal arrow is an isomorphism.

Definition 4.12. We will consider the following definitions in (4.14).

(1) Let $\mathcal{T}$ be a triangulated category with small sums, and let $K$ be an object. We say that $K$ is compact if the functor

$$
\operatorname{Hom}_{\mathcal{T}}(K,-)
$$

commutes with small sums. 
(2) Let $\mathcal{T}$ be a triangulated category, and let $\mathcal{F}$ be a family of objects of $\mathcal{T}$. We say that $\mathcal{F}$ generates $\mathcal{T}$ if the family of functors

$$
\operatorname{Hom}_{\mathcal{T}}(K,-)
$$

for $K \in \mathcal{F}$ is conservative.

Definition 4.13. Let $\mathcal{D}$ be one of $\mathscr{S}$ and $\mathscr{S}^{s m}$, and let

$$
H: \mathcal{D} \rightarrow \operatorname{Tr}^{\otimes}
$$

be a $S m$-premotivic pseudofunctor. For an object $S$ of $\mathcal{D}$, we denote by $1_{S}(1)$ the cone of the object $p_{\sharp} a_{*} 1_{S}[-2]$ where $p: \mathbf{A}_{S}^{1} \rightarrow S$ denotes the projection and $a: S \rightarrow \mathbf{A}_{S}^{1}$ denotes the zero section. Then for any object $K$ of $H(S)$ and positive integer $n$, put

$$
K(n)=K \otimes_{S} 1_{S}(1) \otimes_{S} \cdots \otimes_{S} 1_{S}(1)
$$

where the number of $1_{S}(1)$ is $n$. When there is an object $1_{S}(-1)$ of $H(S)$ such that $1_{S}(1) \otimes 1_{S}(-1) \cong$ $1_{S}$, put

$$
K(-n)=K \otimes_{S} 1_{S}(-1) \otimes_{S} \cdots \otimes_{S} 1_{S}(-1)
$$

where the number of $1_{S}(-1)$ is $n$.

Definition 4.14. Let $\mathcal{D}$ be one of $\mathscr{S}$ and $\mathscr{S}^{s m}$, and let

$$
H: \mathcal{D} \rightarrow \operatorname{Tri}^{\otimes}
$$

be a $S m$-premotivic pseudofunctor. Consider the following axioms.

(B-5) For any object $S$ of $\mathcal{D}$, the counit

$$
p_{\sharp} p^{*} \stackrel{a d^{\prime}}{\longrightarrow} \text { id }
$$

is an isomorphism where $p$ denotes the projection $\mathbb{A}^{1} \times S \rightarrow S$.

(B-6) For any object $S$ of $\mathcal{D}, 1_{S}(1)$ is $\otimes$-invertible, i.e., there is an object $K$ of $H(S)$ such that $1_{S}(1) \otimes_{S} K \cong 1_{S}$.

(B-7) Assume (B-6). For any object $S$ of $\mathcal{D}$, the family of objets $\mathcal{F}_{S}$ generates $H(D)$. Here, $\mathcal{F}_{S}$ denotes the family of objects $f_{\sharp} 1_{X}(d)[n]$ for smooth morphism $f: X \rightarrow S$ and $(d, n) \in \mathbb{Z} \times \mathbb{Z}$.

(B-8) Assume (B-6). For any morphism $f: X \rightarrow S$ of $\mathcal{D}$ and $(d, n) \in \mathbb{Z} \times \mathbb{Z}, f_{\sharp} 1_{X}(d)[n]$ is compact.

(B-9) For any proper morphism $f$ in $\mathcal{D}, f_{*}$ has a right adjoint, denoted by $f$ !

Following [CD12, 2.4.45], we say that $H$ is motivic if it satisfies (Loc) and the axioms (B-5), (B-6), and $(\mathrm{B}-9)$.

Remark 4.15. In (4.14), if $H$ satisfies (Loc), (B-5), (B-6), (B-7), and (B-8), then $H$ satisfies (B-9) by [CD12, 2.4.47], which means that $H$ is motivic.

4.16. Under the notations and hypotheses of (4.14), if $H$ is motivic, then by [CD12, 2.4.50], $H$ satisfies the Grothendieck six operations formalism in [loc. cit]. 


\section{Proof of (1.4), part II}

5.1. Under the notations and hypotheses of (4.4), consider the diagram

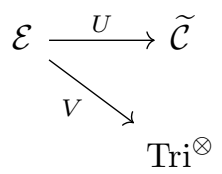

of 2-categories where $V$ is a $\mathscr{P}^{\prime}$-premotivic pseudofunctor satisfying (Loc). One purpose of this section is to show that the diagram can be extended to a commutative diagram

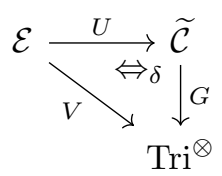

of 2-categories where $G$ is a $\widetilde{P}$-premotivic pseudofunctor satisfying (Loc) and $\delta: V \rightarrow G \circ U$ is a $\mathscr{P}^{\prime}$-premotivic pseudonatural transformation.

Definition 5.2. Under the notations and hypotheses of (4.4), for any closed immersion $i_{1}: Z_{1} \rightarrow$ $S_{1}$, we denote by $\rho_{i_{1}}$ the closed immersion in $\widetilde{\mathcal{C}}$ that is isomorphic to a commutative diagram

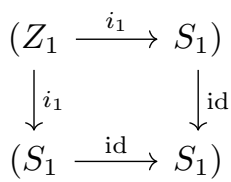

in $\mathcal{C}$.

5.3. Under the notations and hypotheses of (5.1) , for any object $\left(Z_{1} \stackrel{i_{1}}{\rightarrow} S_{1}\right)$ of $\widetilde{\mathcal{C}}$, put $G\left(Z_{1} \rightarrow S_{1}\right)$ as the full subcategory of $V\left(S_{1}\right)$ consisting of objects $K$ such that $j_{1}^{*} K=0$ where $j_{1}: U_{1} \rightarrow S_{1}$ denotes the complement of $i_{1}$. Since $j_{1}^{*}$ is triangulated, $G\left(Z_{1} \rightarrow S_{1}\right)$ is also triangulated, and since $j_{1}^{*}$ is monoidal, $G\left(Z_{1} \rightarrow S_{1}\right)$ has the symmetric closed monoidal structure induced from that of $V\left(S_{1}\right)$.

Then we denote by $\rho_{i_{1} *}$ the embedding

$$
G\left(Z_{1} \rightarrow S_{1}\right) \rightarrow V\left(S_{1}\right) .
$$

Now, we will prove some results about $\rho_{i_{1} *}$ as follows.

Proposition 5.4. Under the notations and hypotheses of (5.3),

(1) the functor $\rho_{i_{1} *}$ has a left adjoint, denoted by $\rho_{i_{1}}^{*}$, and the functor $\rho_{i_{1} *}$ has a right adjoint, denoted by $\rho_{i_{1}}^{!}$,

(2) if we denote by $j_{1}$ the complement of $i_{1}$, then we have a distinguished triangle

$$
j_{1 \sharp} j_{1}^{*} \stackrel{a d}{\longrightarrow} \mathrm{id} \stackrel{a d^{\prime}}{\longrightarrow} \rho_{i_{1} *} \rho_{i_{1}}^{*} \longrightarrow j_{1 \sharp} j_{1}^{*}[1],
$$


(3) the counit

$$
\rho_{i_{1}}^{*} \rho_{i_{1} *} \stackrel{a d^{\prime}}{\longrightarrow} \text { id }
$$

is an isomorphism,

(4) $\rho_{i_{1}}^{*} j_{1 \sharp}=0$.

Proof. Let us first show that $\rho_{i_{1} *}$ has a right adjoint denoted by $\rho_{i_{1}}^{*}$. For any object $K$ of $V\left(S_{1}\right)$, choose a cone of the counit

$$
j_{1 \sharp} j_{1}^{*} K \stackrel{a d^{\prime}}{\longrightarrow} K
$$

in $V\left(S_{1}\right)$, and it is denoted by $\rho_{i_{1}}^{*} K$. Since $j_{1}^{*} \rho_{i_{1}}^{*} K=0$ by 4.8(4)), $\rho_{i_{1}}^{*} K$ is in $G\left(Z_{1} \rightarrow S_{1}\right)$. We also fix a distinguished triangle

$$
j_{1 \sharp} j_{1}^{*} K \stackrel{a d^{\prime}}{\longrightarrow} K \longrightarrow \rho_{i_{1} *} \rho_{i_{1}}^{*} K \longrightarrow j_{1 \sharp} j_{1}^{*} K[1]
$$

in $V\left(S_{1}\right)$, and we denote by ad (resp. $\delta_{K}$ ) the second arrow (resp. third arrow).

Then let us define $\rho_{i_{1}}^{*} a$ for any morphism $a: K \rightarrow L$ in $V\left(S_{1}\right)$. Consider the commutative diagram

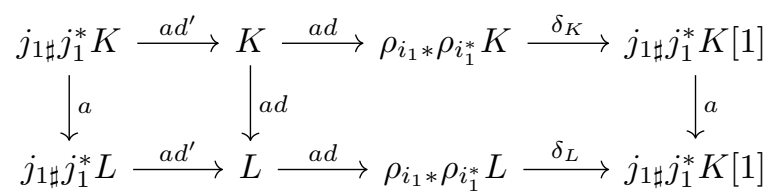

in $V\left(S_{1}\right)$ where the rows are the distinguished triangles. We will show that there is a unique morphism

$$
b: \rho_{i_{1} *} \rho_{i_{1}^{*}} K \longrightarrow \rho_{i_{1} *} \rho_{i_{1}^{*}} L
$$

in $V\left(S_{1}\right)$ making the above diagram commutative. This will be the definition of $\rho_{i_{1}}^{*} a$, and the functoriality of $\rho_{i_{1}}^{*}$ follows from the uniqueness.

The existence of $b$ is obtained by an axiom of triangulated categories, so the remaining is the uniqueness of $b$. If we have two morphisms

$$
b, b^{\prime}: \rho_{i_{1} *} \rho_{i_{1}^{*}} K \longrightarrow \rho_{i_{1} *} \rho_{i_{1}^{*}} L
$$

making the above diagram commutative, put $c=b-b^{\prime}$. Since $c \circ a d=0$, we have $c=d \delta_{K}$ for some morphism

$$
d: j_{1 \sharp j_{1}^{*}} K[1] \rightarrow \rho_{i_{1} *} \rho_{i_{1}}^{*} L
$$

in $V\left(S_{1}\right)$. Hence to show $b=b^{\prime}$, it suffices to show

$$
\operatorname{Hom}_{V\left(S_{1}\right)}\left(j_{1 \sharp} j_{1}^{*} K[1], \rho_{i_{1} *} \rho_{i_{1}}^{*} L\right)=0,
$$

which is true since $j_{1}^{*} \rho_{i_{1} *}=0$.

Thus $b$ is unique, so $\rho_{i_{1}}^{*}$ has a structure of functor. We also obtain the fact that $a d:$ id $\longrightarrow \rho_{i *} \rho_{i}^{*}$ is functorial. Now, we will show that $\rho_{i_{1}}^{*}$ is left adjoint to $\rho_{i_{1} *}$. For any object $K$ of $G\left(Z_{1} \rightarrow S_{1}\right)$, the morphism

$$
\rho_{i_{1} *} K \stackrel{a d}{\longrightarrow} \rho_{i_{1} *} \rho_{i_{1}}^{*} \rho_{i_{1} *} K
$$


is an isomorphism since its cone is $j_{1 \sharp} j_{1}^{*} \rho_{i_{1} *} K[1]$, which is zero. Since $\rho_{i_{1} *}$ is fully faithful, the inverse of the above morphism induces the isomorphism

$$
\rho_{i_{1}}^{*} \rho_{i_{1} *} K \longrightarrow K
$$

in $G\left(Z_{1} \rightarrow S_{1}\right)$. It is denoted by $a d^{\prime}$. Since $a d$ is functorial, $a d^{\prime}$ is also functorial. From construction, the compositions

$$
\begin{gathered}
\rho_{i_{1}}^{*} \stackrel{a d}{\longrightarrow} \rho_{i_{1}}^{*} \rho_{i_{1} *} \rho_{i_{1}}^{*} \stackrel{a d^{\prime}}{\longrightarrow} \rho_{i_{1}}^{*} \\
\rho_{i_{1} *} \stackrel{a d}{\longrightarrow} \rho_{i_{1} *} \rho_{i_{1}}^{*} \rho_{i_{1} *} \stackrel{a d^{\prime}}{\longrightarrow} \rho_{i_{1} *}
\end{gathered}
$$

are isomorphisms. Thus $\rho_{i_{1}}^{*}$ is right adjoint to $\rho_{i_{1} *}$. By a similar method, $\rho_{i_{1} *}$ has a left adjoint, denoted by $\rho_{i_{1}}^{!}$.

Applying $\rho_{i_{1}}^{*}$ to the distinguished triangle (5.4.1) and using the fact that the natural transformation

$$
\rho_{i_{1}}^{*} \rho_{i_{1} *} \stackrel{a d^{\prime}}{\longrightarrow} \mathrm{id}
$$

is an isomorphism, we have $\rho_{i_{1}}^{*} j_{1 \sharp}=0$.

5.5. Under the notations and hypotheses of $(\underline{5.3})$, consider a morphism $\widetilde{X}_{2} \stackrel{f_{1}}{\rightarrow} \widetilde{X_{1}}$ given by a commutative diagram

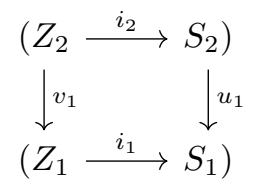

in $\mathcal{C}$. We denote by $j_{1}$ (resp. $j_{2}$ ) the complement of $i_{1}$ (resp. $i_{2}$ ). Then we have the commutative diagram

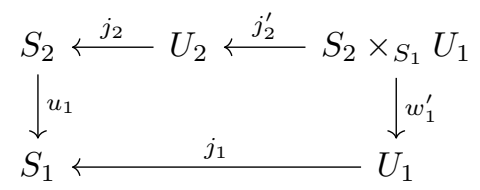

in $\mathcal{C}$ where $w_{1}^{\prime}$ denotes the projections and $j_{2}^{\prime}$ is obtained by (D-4). By (B-3) and (5.4), we have

$$
\rho_{i_{2}}^{*} u_{1}^{*} j_{1 \sharp} \cong \rho_{i_{2}}^{*} j_{2 \sharp} j_{2 \sharp}^{\prime} w_{1}^{\prime *}=0 .
$$

Thus the natural transformation

$$
\rho_{i_{2}}^{*} u_{1}^{*} \stackrel{a d}{\longrightarrow} \rho_{i_{2}}^{*} u_{1}^{*} \rho_{i_{1} *} \rho_{i_{1}}^{*}
$$

is an isomorphism since its cone is 0 by (5.4).

When (5.5.1) is Cartesian, we have the Cartesian diagram

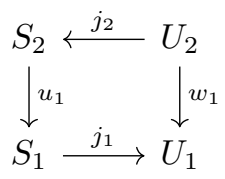


in $\mathcal{C}$. Then we have

$$
j_{2}^{*} u_{1}^{*} \rho_{i_{1} *} \cong w_{1}^{*} j_{1}^{*} \rho_{i_{1} *}=0 .
$$

Thus by (5.4), the natural transformation

$$
u_{1}^{*} \rho_{i_{1} *} \stackrel{a d}{\longrightarrow} \rho_{i_{2} *} \rho_{i_{2}}^{*} u_{1}^{*} \rho_{i_{1} *}
$$

is an isomorphism. Thus we have proven the following result.

Proposition 5.6. Under the notations and hypotheses of (5.5),

(1) the natural transformation

$$
\rho_{i_{2}}^{*} u_{1}^{*} \stackrel{a d}{\longrightarrow} \rho_{i_{2}}^{*} u_{1}^{*} \rho_{i_{1} *} \rho_{i_{1}}^{*}
$$

is an isomorphism,

(2) when (5.5.1) is Cartesian, the natural transformation

$$
u_{1}^{*} \rho_{i_{1} *} \stackrel{a d}{\longrightarrow} \rho_{i_{2} *} \rho_{i_{2}}^{*} u_{1}^{*} \rho_{i_{1} *}
$$

is an isomorphism.

5.7. Now, we will construct $G$ introduced in (5.1). Under the notations and hypotheses of (5.3), for any morphism $\widetilde{f}_{1}$ in $\mathscr{C}$ given by a commutative diagram

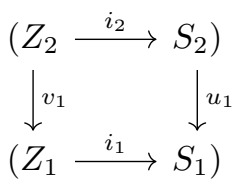

in $\mathcal{C}$, put

$$
G\left(\widetilde{f}_{1}\right):=\rho_{i_{2}}^{*} u_{1}^{*} \rho_{i_{1} *} .
$$

It is also denoted by $\widetilde{f}_{1}{ }^{*}$ following the convention in (2.2). We will first construct a monoidal structure for $G\left(\widetilde{f_{1}}\right)$. Since $u_{1}^{*}$ and $\rho_{i_{1} *}$ are monoidal, it suffices to construct a monoidal structure for $\rho_{i_{2}}^{*}$. Let $j_{2}: U_{2} \rightarrow S_{2}$ denotes the complement of $i_{2}$, and let $K$ and $L$ be objects of $V\left(S_{2}\right)$. Consider the distinguished triangle

$$
\rho_{i_{2} *} \rho_{i_{2}}^{*} K \otimes_{S_{2}} j_{2 \sharp} j_{2}^{*} L \stackrel{a d^{\prime}}{\longrightarrow} \rho_{i_{2} *} \rho_{i_{2}}^{*} K \otimes_{S_{2}} L \stackrel{a d}{\longrightarrow} \rho_{i_{2} *} \rho_{i_{2}}^{*} K \otimes_{S_{2}} \rho_{i_{2} *} \rho_{i_{2}}^{*} L \longrightarrow \rho_{i_{2} *} \rho_{i_{2}}^{*} K \otimes_{S_{2}} j_{2 \sharp} j_{2}^{*} L[1] .
$$

in $V\left(S_{2}\right)$. The first object is zero by (B-4) for $V$ since $j_{2}^{*} \rho_{i_{2} *}=0$. Thus the natural transformation

$$
\rho_{i_{2} *} \rho_{i_{2}}^{*} K \otimes_{S_{2}} L \stackrel{a d}{\longrightarrow} \rho_{i_{2} *} \rho_{i_{2}}^{*} K \otimes_{S_{2}} \rho_{i_{2} *} \rho_{i_{2}}^{*} L
$$

is an isomorphism. Then consider the commutative diagram

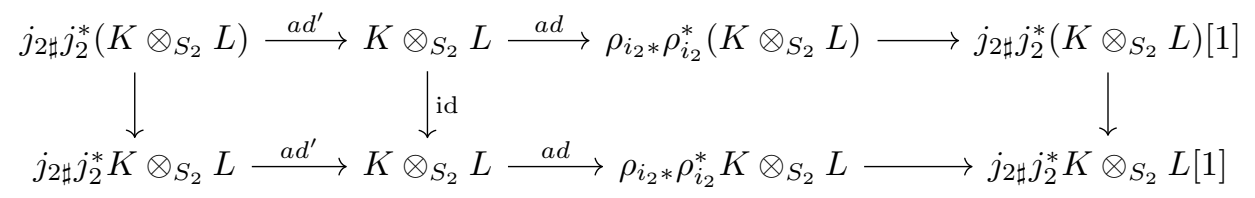


in $V\left(S_{2}\right)$ where the left side and the right side vertical arrows are given by

$$
j_{2 \sharp} j_{2}^{*}\left(K \otimes_{S_{2}} L\right) \stackrel{\sim}{\longrightarrow} j_{2 \sharp}\left(j_{2}^{*} K \otimes_{U_{2}} j_{2}^{*} L\right) \stackrel{E x}{\longrightarrow} j_{2 \sharp} j_{2}^{*} K \otimes_{S_{2}} L .
$$

By the proof of (5.4) (more precisely, the proof of uniqueness of $b$ in (loc. cit)), there is a unique isomorphism

$$
\rho_{i_{2} *} \rho_{i_{2}}^{*}\left(K \otimes S_{2} L\right) \longrightarrow \rho_{i_{2} *} \rho_{i_{2}}^{*} K \otimes_{S_{2}} L
$$

in $V\left(S_{1}\right)$ making the above diagram commutes. With (5.7.1), we obtain the isomorphism

$$
\rho_{i_{2} *} \rho_{i_{2}}^{*}\left(K \otimes_{S_{2}} L\right) \longrightarrow \rho_{i_{2} *} \rho_{i_{2}}^{*} K \otimes_{S_{2}} \rho_{i_{2} *} \rho_{i_{2}}^{*} L
$$

in $V\left(S_{1}\right)$. Since $\rho_{i_{2} *}$ is monoidal and fully faithful, we obtain the isomorphism

$$
\rho_{i_{2}}^{*}\left(K \otimes_{S_{2}} L\right) \longrightarrow \rho_{i_{2}}^{*} K \otimes_{Z_{2}} \rho_{i_{2}}^{*} L
$$

in $G\left(\widetilde{X_{2}}\right)$. This satisfies the coherence condition by the proof of (5.4) again.

5.8. Let us continue the argument in (5.7). We will show that $G$ has a contravariant pseudofunctor structure. For any object $\widetilde{X_{1}}=\left(Z_{1} \stackrel{i_{1}}{\rightarrow} S_{1}\right)$ in $\widetilde{\mathcal{C}}$, consider the identity morphism id ${\widetilde{X_{1}}}_{\widetilde{X_{1}}} \rightarrow \widetilde{X_{1}}$ in $\widetilde{\mathcal{C}}$. The natural transformation

$$
G_{\mathrm{id}_{\widetilde{X_{1}}}}: \operatorname{id}_{\widetilde{X}_{1}}^{*} \longrightarrow \mathrm{id}
$$

is given by

$$
\rho_{i_{1}}^{*} \rho_{i_{1} *} \stackrel{a d^{\prime}}{\longrightarrow} \mathrm{id},
$$

which is an isomorphism by (5.4). For any morphisms $\widetilde{X_{3}} \stackrel{f_{2}}{\longrightarrow} \widetilde{X_{2}} \stackrel{f_{1}}{\longrightarrow} \widetilde{X_{1}}$ in $\widetilde{\mathcal{C}}$ given by a diagram

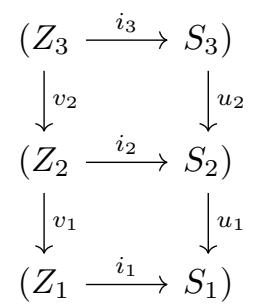

in $\mathcal{C}$, the natural isomorphism

$$
G_{f_{1}, f_{2}}:{\widetilde{f_{2}}}^{*}{\widetilde{f_{1}}}^{*} \longrightarrow\left(\widetilde{f}_{1} \widetilde{f}_{2}\right)^{*}
$$

is given by

$$
\rho_{i_{3}}^{*} u_{2}^{*} \rho_{i_{2} *} \rho_{i_{2}}^{*} u_{1}^{*} \rho_{i_{1} *} \stackrel{a d^{-1}}{\longrightarrow} \rho_{i_{3}}^{*} u_{2}^{*} u_{1}^{*} \rho_{i_{1} *} \stackrel{\sim}{\longrightarrow} \rho_{i_{3}}^{*}\left(u_{1} u_{2}\right)^{*} \rho_{i_{1} *} .
$$

Here, the first arrow is defined and an isomorphism by (5.6). Now, we will verify the axioms of psedofunctors for $G$ as follows.

(1) Consider morphisms

$$
\widetilde{X_{2}} \stackrel{f_{1}}{\longrightarrow} \widetilde{X_{1}} \stackrel{\mathrm{id}_{\widetilde{X_{1}}}}{\longrightarrow} \widetilde{X_{1}}
$$


in $\mathscr{C}$ given by a commutative diagram

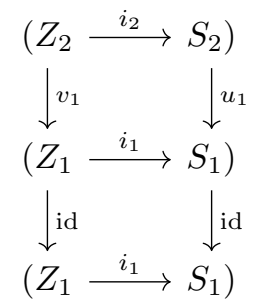

in $\mathcal{C}$. We have to show that the diagram

$$
\begin{aligned}
& \widetilde{f}_{1}^{*} \operatorname{id}_{\widetilde{X}_{1}}^{*} \stackrel{G_{\mathrm{id}}}{\longrightarrow} \widetilde{f}_{1}{ }^{*} \\
& \downarrow G_{\mathrm{id}_{\widetilde{X_{1}}}, f_{1}}|| \\
& \left(\operatorname{id}_{\widetilde{X_{1}}} \widetilde{f}_{1}\right)^{*}=\widetilde{f}_{1}^{*}
\end{aligned}
$$

of functors commutes. It is the diagram

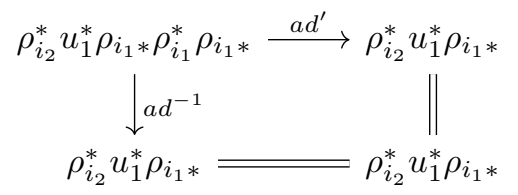

of functors, which commutes.

(2) Consider morphisms $\widetilde{X_{2}} \stackrel{\text { id }_{\widetilde{X_{2}}}}{\longrightarrow} \widetilde{X_{2}} \stackrel{f_{1}}{\longrightarrow} \widetilde{X_{1}}$ in $\widetilde{C}$. As in the above argument, the diagram

$$
\begin{gathered}
\operatorname{id}_{\widetilde{X_{2}}}^{*} f_{1}^{*} \stackrel{G_{\mathrm{id}}}{\stackrel{\bar{X}_{2}}{\longrightarrow}} \widetilde{f}_{1}^{*} \\
\left.\right|_{f_{1}, \mathrm{id} \widetilde{X_{2}}} \\
\left(f_{1} \mathrm{id}_{\widetilde{X_{2}}}\right)^{*}=f_{1}^{*}
\end{gathered}
$$

of functors commutes.

(3) Consider morphisms

$$
\widetilde{X_{4}} \stackrel{\widetilde{f_{3}}}{\rightarrow} \widetilde{X_{3}} \stackrel{\widetilde{f_{2}}}{\rightarrow} \widetilde{X_{2}} \stackrel{\widetilde{f_{1}}}{\rightarrow} \widetilde{X_{1}}
$$


in $\mathcal{C}$ given by a commutative diagram

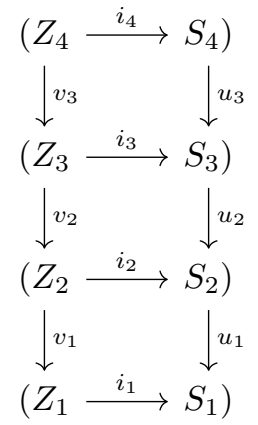

in $\mathcal{C}$. We have to show that the diagram

$$
\begin{aligned}
& \widetilde{f}_{3}{ }^{*} \widetilde{f}_{2}{ }^{*} \widetilde{f}_{1}{ }^{*} \stackrel{G_{\widetilde{f_{1}}, \widetilde{f}_{2}}}{\longrightarrow} \widetilde{f}_{3}{ }^{*}\left(\widetilde{f}_{1} \widetilde{f}_{2}\right)^{*} \\
& \downarrow G_{\widetilde{f_{2}}, \widetilde{f_{3}}} \downarrow G_{\widetilde{f_{1}} \widetilde{f_{2}}, \widetilde{f_{3}}} \\
& \left(\widetilde{f}_{2} \widetilde{f}_{3}\right)^{*} \widetilde{f}_{1} \stackrel{G_{\widetilde{f_{1}}}, \widetilde{f}_{2} \widetilde{f}_{3}}{\longrightarrow}\left(\widetilde{f}_{1} \widetilde{f}_{2} \widetilde{f}_{3}\right)^{*}
\end{aligned}
$$

of functors commutes. It is the big outside diagram of the diagram

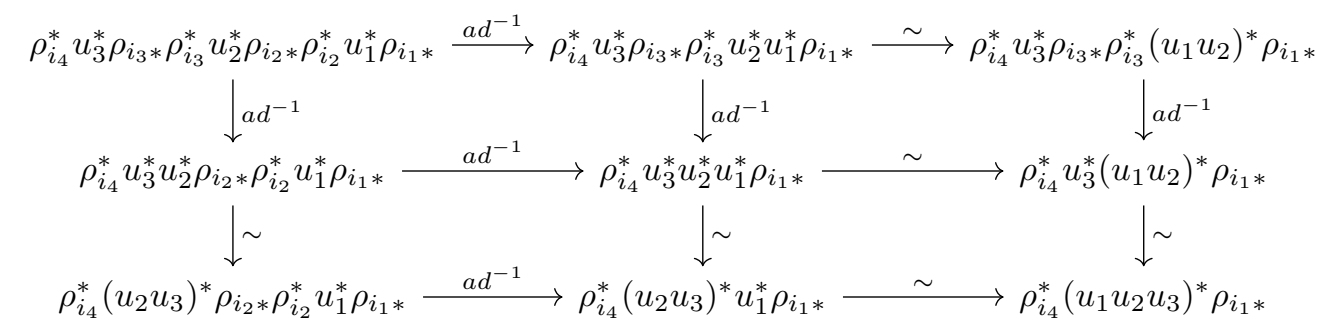

of functors, whose small diagrams commute. Here, the arrows denoted by $a d^{-1}$ are defined and isomorphisms by (5.6). Thus (5.8.1) commutes.

Thus we have verified all axioms of pseudofunctors for $G$, so $G$ is a pseudofunctor.

5.9. Let us interpret $[5.6(2))$ as follows. Consider a morphism $\widetilde{f_{1}}: \widetilde{X_{2}} \rightarrow \widetilde{X_{1}}$ given by a Cartesian diagram

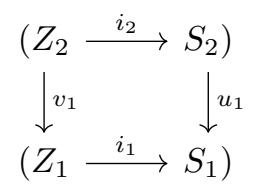

in $\mathcal{C}$. Then we have the Cartesian diagram

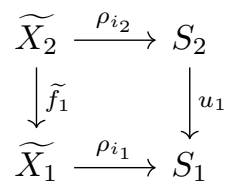


in $\widetilde{\mathcal{C}}$. Using $G$ constructed in (5.8), we have the exchange transformation

$$
u_{1}^{*} \rho_{i_{1} *} \stackrel{E x}{\longrightarrow} \rho_{i_{2} *} \widetilde{f}_{1}^{*}
$$

is given by

$$
u_{1}^{*} \rho_{i_{1} *} \stackrel{a d}{\longrightarrow} \rho_{i_{2} *} \rho_{i_{2}}^{*} u_{1}^{*} \rho_{i_{1} *} \stackrel{G_{u_{1}, \rho_{i_{2}}}}{\longrightarrow} \rho_{i_{2} *}{\widetilde{f_{1}}}_{1}^{*} \rho_{i_{1}}^{*} \rho_{i_{1} *} \stackrel{a d^{\prime}}{\longrightarrow} \rho_{i_{2}}^{*} \widetilde{f}_{1}^{*} .
$$

The first arrow is an isomorphism by $[5.6(2))$, and the second arrow is an isomorphism since $G$ is a pseudofunctor. The third arrow is also an isomorphism by transformation is an isomorphism.

5.10. Let us continue the argument in (5.8). So far, we have constructed the contravariant pseudofunctor $G$. Now, we will show that $G$ is $\widetilde{\mathscr{P}}$-premotivic by verifying the axioms from (B-1) to (B-4) as follows.

(1) For any morphism $\widetilde{f}_{1}$ given by a commutative diagram

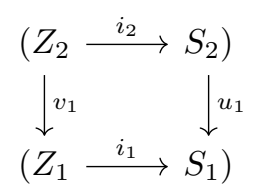

in $\mathcal{C}$, the right adjoint of ${\widetilde{f_{1}}}_{1}^{*}$ is $\rho_{i_{1}}^{!} u_{1 *} \rho_{i_{2} *}$. Thus (B-1) holds for $G$.

(2) For any $\widetilde{\mathscr{P}}$-morphism $\widetilde{f}_{1}$ in $\widetilde{C}$ given by a Cartesian diagram

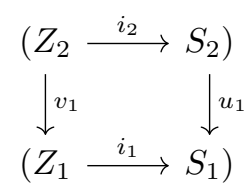

in $\mathcal{C}$, we will show that the left adjoint of $\widetilde{f}_{1}^{*}$ is $\rho_{i_{1}}^{*} u_{1 \sharp} \rho_{i_{2} *}$. For this, we will show that $\widetilde{f}_{1}^{*}$ is isomorphic to

$$
\rho_{i_{2}}^{!} u_{1}^{*} \rho_{i_{1} *}
$$

Consider the Cartesian diagram

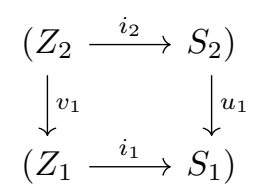

where $j_{1}$ (resp. $j_{2}$ ) denotes the complement of $i_{1}$ (resp. $i_{2}$ ). Since the natural transformations

$$
\rho_{i_{2}}^{*} \rho_{i_{2} *} \stackrel{a d^{\prime}}{\longrightarrow} \text { id, } \quad \text { id } \stackrel{a d}{\longrightarrow} \rho_{i_{2}}^{!} \rho_{i_{2} *}
$$

are isomorphisms by $[5.4(3))$, we only need to show that the essential image of $u_{1}^{*} \rho_{i_{1} *}$ is in $G\left(Z_{2} \rightarrow S_{2}\right)$, which is true by (5.9). Thus (B-2) holds for $G$. 
(3) Consider a Cartesian diagram

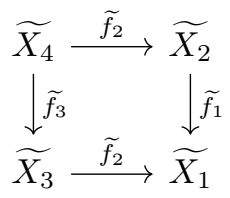

in $\widetilde{C}$ given by a commutative diagram

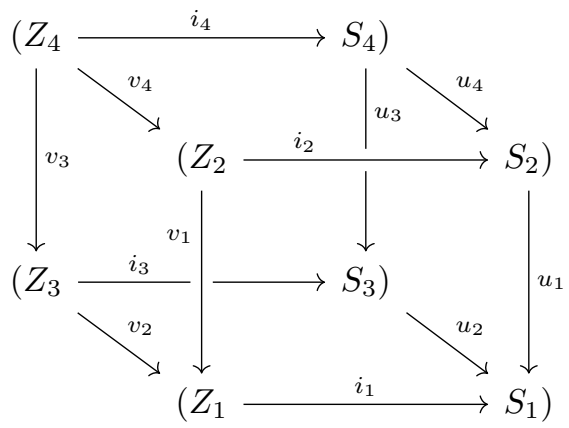

in $\mathcal{C}$. Assume that $\widetilde{f}_{1}$ is a $\widetilde{\mathscr{P}}$-morphism. Consider the natural transformation

$$
\widetilde{f}_{1}^{*} \widetilde{f}_{2 *} \stackrel{E x}{\longrightarrow} \widetilde{f}_{4 *} \widetilde{f}_{3}^{*}
$$

that is the left adjoint of the exchange transformation in (B-3). We will show that this is an isomorphism. By (5.5(3)), $\rho_{i_{2} *}$ is fully faithful, so it suffices to show that the natural transformation

$$
\rho_{i_{2} *} \widetilde{f}_{1}^{*} \widetilde{f}_{2_{*}} \stackrel{E x}{\longrightarrow} \rho_{i_{2} *} \widetilde{f}_{4_{*}} \widetilde{f}_{3}^{*}
$$

is an isomorphism.

From the commutative diagram

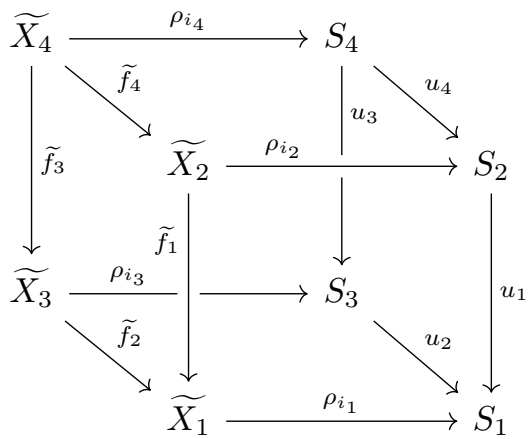

in $\widetilde{\mathcal{C}}$, we have the commutative diagram

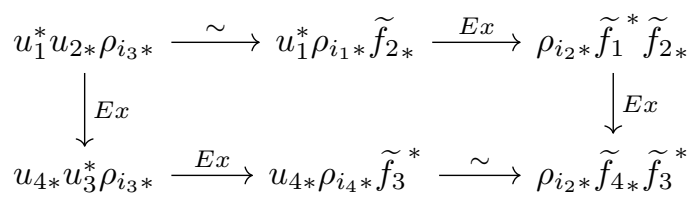


of functors. The left vertical arrow is an isomorphism by (B-3) for $V$. Hence to show that the right vertical arrow is an isomorphism, it suffices to show that the upper right horizontal and lower left horizontal arrows are isomorphisms. This follows from (5.9). Thus (B-3) holds for $G$.

(4) Let $\widetilde{f_{1}}: \widetilde{X_{2}} \rightarrow \widetilde{X_{1}}$ be a $\widetilde{\mathscr{P}}$-morphism in $\widetilde{C}$ given by a Cartesian diagram

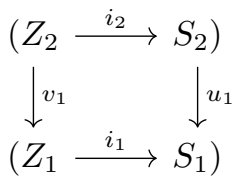

in $\mathcal{C}$. For any object $K$ of $G\left(\widetilde{X_{2}}\right)$ and $L$ of $G\left(\widetilde{X_{1}}\right)$, we will show that the exchange transformation

$$
\widetilde{f}_{1_{\sharp}}\left(K \otimes_{\widetilde{X}_{2}} \widetilde{f}_{1}^{*} L\right) \stackrel{E x}{\longrightarrow} \widetilde{f}_{1_{\sharp}} K \otimes_{\widetilde{X}_{1}} L
$$

is an isomorphism. Since $\rho_{i_{1}}^{*}$ and $\rho_{i_{2}}^{*}$ are essentially surjective by $\left.\underline{(5.4}(3)\right)$, we can put

$$
K=\rho_{i_{2}}^{*} K^{\prime}, \quad L=\rho_{i_{1}}^{*} L^{\prime} .
$$

Then consider the commutative diagram

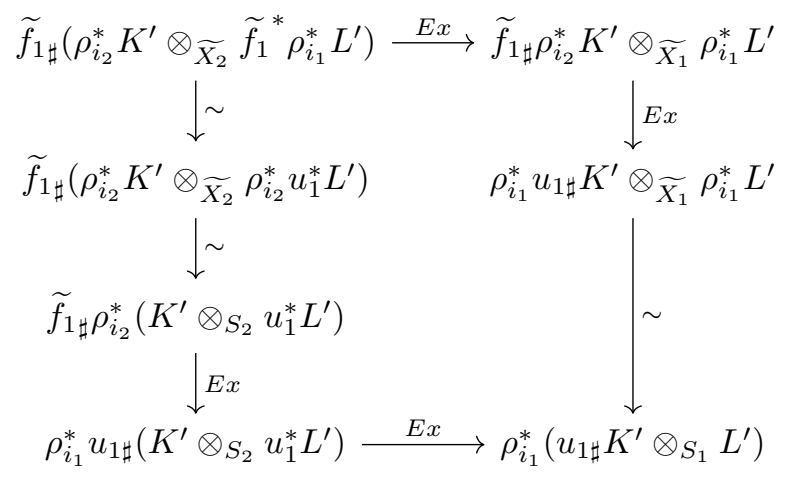

in $G\left(\widetilde{X_{1}}\right)$. Here, the middle left vertical and the lower right vertical arrows are obtained by the fact that $\rho_{i_{1}}^{*}$ and $\rho_{i_{2}}^{*}$ are monoidal, which is proved in (5.7). Since the bottom left vertical arrow and the upper right vertical arrows are isomorphisms by (B-3), to show that the upper horizontal arrow is an isomorphism, it suffices to show that the lower horizontal arrow is an isomorphism. This follows from (B-4) for $G$.

Thus $G$ is a $\widetilde{\mathscr{P}}$-premotivic pseudofunctor.

5.11. Let us continue the argument in (5.10). We will show that $G$ satisfies (Loc). Recall from (4.4) that a closed immersion in $\widetilde{\mathcal{C}}$ is of the form $\rho_{i_{1}}$ for some closed immersion $i_{1}: Z_{1} \rightarrow S_{1}$ in $\mathcal{C}$. The complement of $\rho_{i_{1}}$ in $\widetilde{\mathcal{C}}$ is $j_{1}$ where $j_{1}: U_{1} \rightarrow S_{1}$ denotes the complement of $i_{1}$ in $\mathcal{C}$. 
If $a: K \rightarrow K^{\prime}$ is a morphism in $G\left(S_{1}\right)$ such that $\rho_{i_{1}}^{*} a$ and $j_{1}^{*} a$ are isomorphisms, then consider the commutative diagram

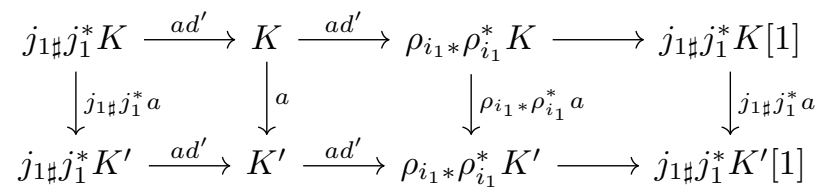

in $G\left(S_{1}\right)$ where the two rows are distinguished triangles obtained by $[5.4(2))$. By assumption, the first and third vertical arrows are isomorphisms, so the second vertical arrow is an isomorphism.

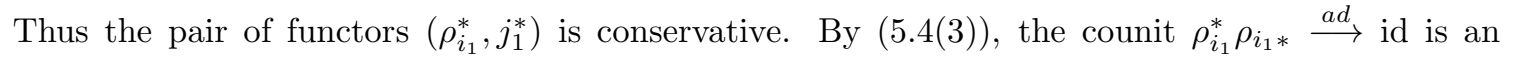
isomorphism. Thus $G$ satisfies $\left(\operatorname{Loc}_{\rho_{i_{1}}}\right)$.

Consider the Cartesian diagram

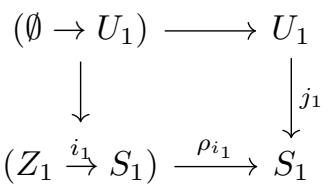

in $\widetilde{\mathcal{C}}$. By the construction of $G$, we have $G\left(\emptyset \rightarrow U_{1}\right)=0$. Thus we have verified all axioms of (Loc) for $G$, so $G$ satisfies (Loc).

5.12. Let us continue the argument in (5.11). We will construct a pseudonatural equivalence $\delta: V \rightarrow G \circ U$ in (5.1.1). Recall from (5.3) that $G\left(U\left(S_{1}\right)\right)=V\left(S_{1}\right)$, and for any object $S_{1}$ of $\mathcal{E}$, put $\delta\left(S_{1}\right)$ as the identity functor

$$
V\left(S_{1}\right) \longrightarrow G\left(U\left(S_{1}\right)\right) \text {. }
$$

For any morphism $f_{1}: S_{2} \rightarrow S_{1}$ in $\mathcal{E}$, put $\delta\left(S_{1}\right)$ as the natural transformation

$$
f_{1}^{*} \stackrel{\sim}{\longrightarrow} \operatorname{id}_{S_{2}}^{*} f_{1}^{*} \operatorname{id}_{S_{1} *} \text {. }
$$

We will verify the axioms of pseudonatural transformations for $\delta$ as follows.

(1) For any object $S_{1}$ of $\mathcal{E}$, we have to show that the diagram

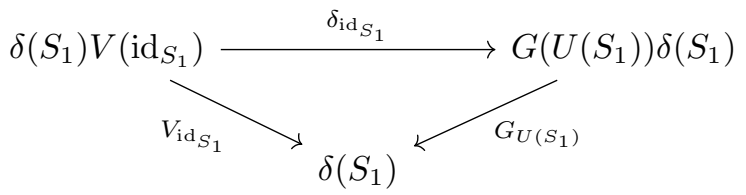

of functors commutes. It is the diagram

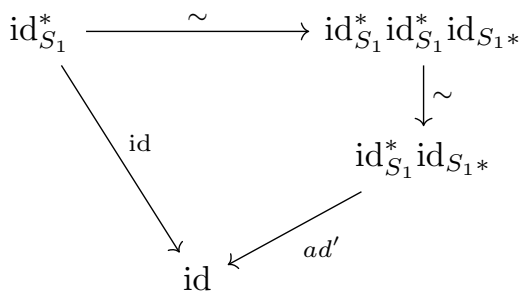

of functors, which commutes. 
(2) For any morphisms $S_{3} \stackrel{f_{2}}{\rightarrow} S_{2} \stackrel{f_{1}}{\rightarrow} S_{1}$ in $\mathcal{E}$, we have to show that the diagram

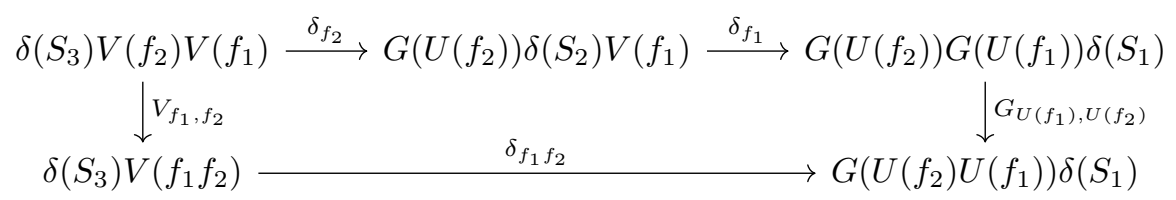

of functors commutes. It is the diagram

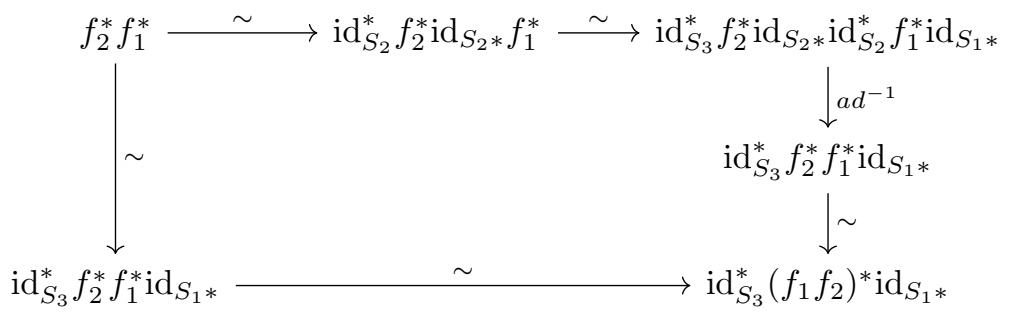

of functors, which commutes.

We have verified all axioms of pseudonatural transformations for $\delta$, and $\delta\left(S_{1}\right)$ is an equivalence for any object $S_{1}$ of $\mathcal{C}$, so $\delta$ is a pseudonatural equivalence.

So far, we have proved the following.

Theorem 5.13. Under the notations and hypotheses of (5.1), there is a $\widetilde{P}$-premotivic pseudofunctor $G: \mathcal{E} \rightarrow \operatorname{Tr}^{\otimes}$ satisfying (Loc) and a pseudonatural equivalence $\delta: V \rightarrow G \circ U$ such that the diagram

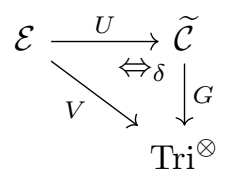

of 2-categories commutes.

5.14. Now, we will prove that the above construction is functorial in the following sense.

Theorem 5.15. Under the notations and hypotheses of (5.1), consider a diagram

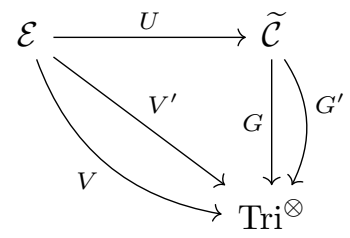

of 2-categories where $V$ and $V^{\prime}$ are $\mathscr{P}^{\prime}$-premotivic pseudofunctors satisfying (Loc) and $G$ and $G^{\prime}$

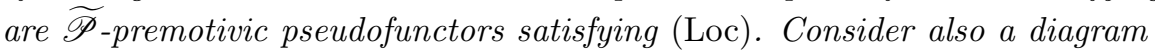

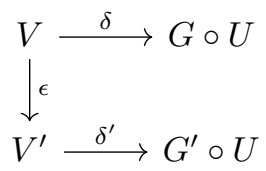


where $\epsilon$ is a $\mathscr{P}^{\prime}$-premotivic pseudonatural transformations and $\delta$ and $\delta^{\prime}$ are pseudonatural equivalences. Then there is a $\widetilde{P}$-premotivic pseudonatural transformation

$$
\beta: G \rightarrow G^{\prime}
$$

unique up to isomorphisms such that the induced $\mathscr{P}^{\prime}$-premotivic pseudonatural transformation $G \circ$ $U \rightarrow G^{\prime} \circ U$ makes the above diagram commutative.

Proof. We may assume that $\delta$ and $\delta^{\prime}$ are the identities. We will first construct $\beta: G \rightarrow G^{\prime}$. For any object $\widetilde{X_{1}}=\left(Z_{1} \stackrel{i_{1}}{\rightarrow} S_{1}\right)$ of $\widetilde{\mathcal{C}}$, put $\beta\left(\widetilde{X_{1}}\right)$ as the composition

$$
G\left(\widetilde{X_{1}}\right) \stackrel{\rho_{i_{1} *}}{\longrightarrow} V\left(S_{1}\right) \stackrel{\epsilon\left(S_{1}\right)}{\longrightarrow} V^{\prime}\left(S_{1}\right) \stackrel{\rho_{i_{1}}^{*}}{\longrightarrow} G^{\prime}\left(\widetilde{X_{1}}\right)
$$

of functors. For any morphism $\widetilde{f_{1}}: \widetilde{X_{2}} \rightarrow \widetilde{X_{1}}$ in $\mathcal{C}$ given by a commutative diagram

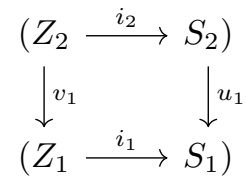

in $\mathcal{C}$, put $\beta\left(\widetilde{f_{1}}\right)$ as the natural transformation

$$
\beta\left(\widetilde{X_{2}}\right) G\left(\widetilde{f_{1}}\right) \longrightarrow G^{\prime}\left(\widetilde{f_{1}}\right) \beta\left(\widetilde{X_{1}}\right)
$$

given by the composition

$$
\rho_{i_{2}}^{*} \epsilon\left(S_{2}\right) \rho_{i_{2} *} \widetilde{f}_{1}^{*} \stackrel{E x^{-1}}{\longrightarrow} \rho_{i_{2}}^{*} \epsilon\left(S_{2}\right) u_{1}^{*} \rho_{i_{1} *} \stackrel{\epsilon_{u_{1}}}{\longrightarrow} \rho_{i_{2}}^{*} u_{1}^{*} \epsilon\left(S_{1}\right) \rho_{i_{1} *} \stackrel{\sim}{\longrightarrow} \widetilde{f}_{1}^{*} \rho_{i_{1}}^{*} \epsilon\left(S_{1}\right) \rho_{i_{1} *} .
$$

Here, the first arrow is defined and an isomorphism by (4.10).

Now, we will verify the axioms of pseudonatural transformations for $\beta$.

(1) For any object $\widetilde{X_{1}}=\left(Z_{1} \stackrel{i_{1}}{\rightarrow} S_{1}\right)$ in $\widetilde{\mathcal{C}}$, we have to show that the diagram

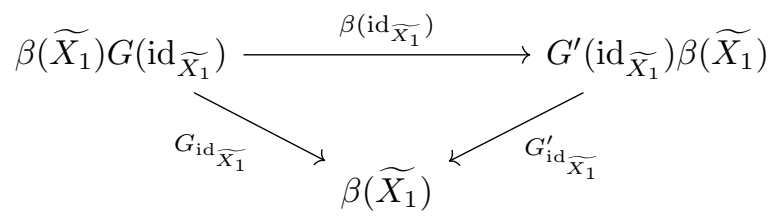

of functors commutes. It is true since it is the big outside diagram of the diagram

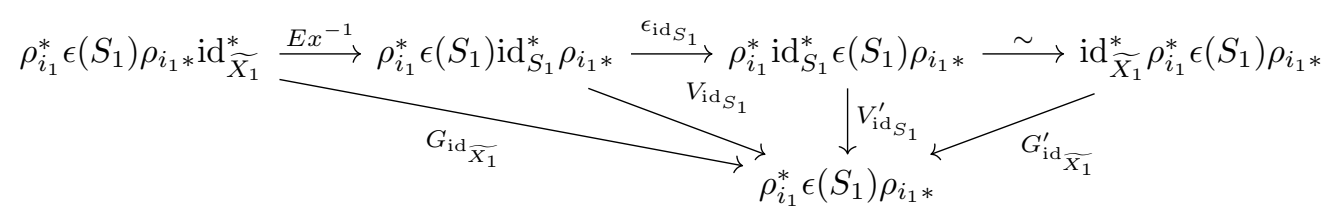

of functors, whose small diagrams commute. 
(2) For any morphisms $\widetilde{X_{3}} \stackrel{\widetilde{f_{2}}}{\longrightarrow} \widetilde{X_{2}} \stackrel{\widetilde{f_{1}}}{\longrightarrow} \widetilde{X_{1}}$ in $\widetilde{\mathcal{C}}$ given by a commutative diagram

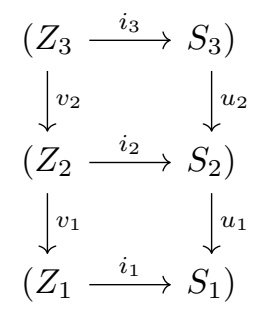

in $\mathcal{C}$, we have to show that the diagram

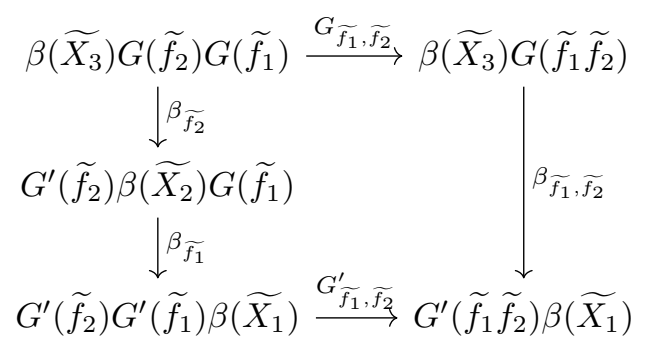

of functors commutes. It is true since it is the big outside diagram of the diagram

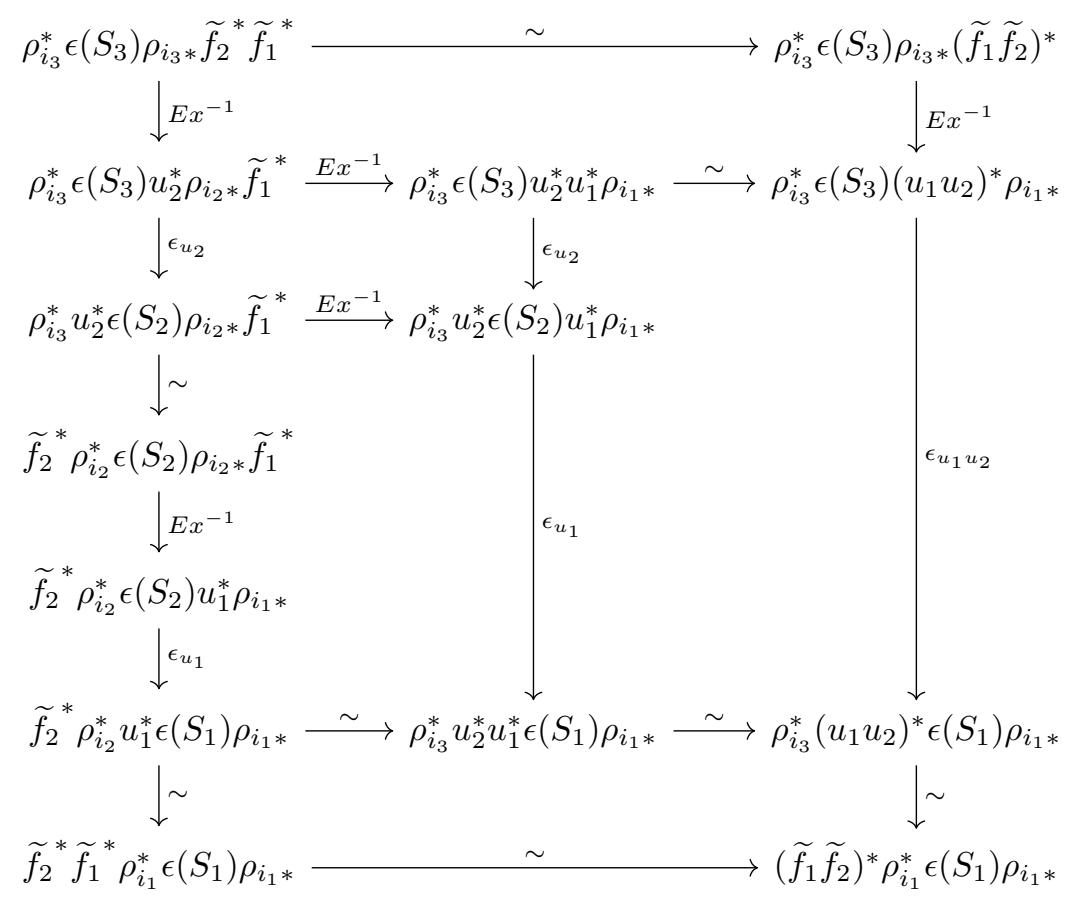

of functors, whose small diagrams commute. 
We have verified all axioms of pseudonatural transformations for $\beta$, so $\beta$ is a pseudonatural transformation. The commutativity of the diagram

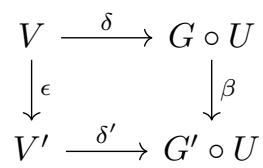

of pseudofunctors follows from construction. Now, we will verify the axioms (C-1) and (C-2) for $\beta$. Let $\widetilde{X_{1}}=\left(Z_{1} \stackrel{i_{1}}{\rightarrow} S_{1}\right)$ be an object of $\widetilde{\mathcal{C}}$. Since the functor $\epsilon\left(S_{1}\right)$ has a right adjoint by $(\mathrm{C}-1)$ for $\epsilon$, the functor

$$
\beta\left(\widetilde{X_{1}}\right)=\rho_{i_{1}}^{*} \epsilon\left(S_{1}\right) \rho_{i_{1}}
$$

has a right adjoint. Thus $\beta$ satisfies $(\mathrm{C}-1)$. Let $f_{1}: \widetilde{X_{2}} \rightarrow \widetilde{X_{1}}$ be a morphism in $\widetilde{P}$ given by a Cartesian diagram

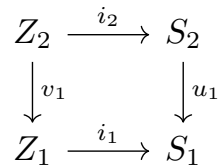

in $\mathcal{C}$. Then we have the Cartesian diagram

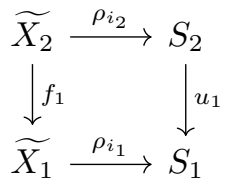

in $\widetilde{\mathcal{C}}$, and we have the commutative diagram

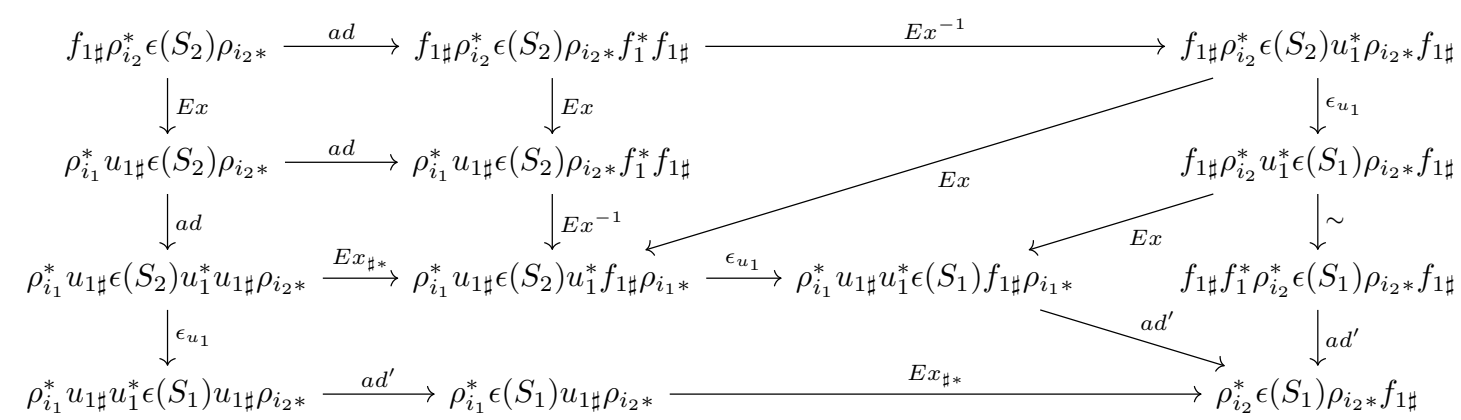

of functors. Here, the arrows denoted by $E x_{\sharp *}$ are induced from (4.11). The composition of the five arrows in the upper right route is the exchange transformation

$$
f_{1 \sharp} \beta\left(S_{2}\right) \stackrel{E x}{\longrightarrow} \beta\left(S_{1}\right) f_{1 \sharp} .
$$

To show that this is an isomorphism, it suffices to show that the composition

$$
\begin{aligned}
f_{1 \sharp} \rho_{i_{2}}^{*} \epsilon\left(S_{2}\right) \rho_{i_{2} *} & \stackrel{E x}{\longrightarrow} \rho_{i_{1}}^{*} u_{1 \sharp} \epsilon\left(S_{2}\right) \rho_{i_{2} *} \stackrel{a d}{\longrightarrow} \rho_{i_{1}}^{*} u_{1 \sharp} \epsilon\left(S_{2}\right) u_{1}^{*} u_{1 \sharp} \rho_{i_{2} *} \stackrel{\epsilon_{u_{1}}}{\longrightarrow} \rho_{i_{1}}^{*} u_{1 \sharp} u_{1}^{*} \epsilon\left(S_{1}\right) u_{1 \sharp} \rho_{i_{2} *} \\
& \stackrel{a d^{\prime}}{\longrightarrow} \rho_{i_{1}}^{*} \epsilon\left(S_{1}\right) u_{1 \sharp} \rho_{i_{2} *} \stackrel{E x_{\sharp *}}{\longrightarrow} \rho_{i_{2}}^{*} \epsilon\left(S_{1}\right) \rho_{i_{2} *} f_{1 \sharp}
\end{aligned}
$$


of the natural transformations in the lower left route is an isomorphism. The composition of the second, third, and fourth arrows is an isomorphism by $(\mathrm{C}-2)$ for $\epsilon$, and the first arrow is an isomorphism by (B-3) for $G^{\prime}$. Since the fifth arrow is an isomorphism by (4.11), (5.15.1) is an isomorphism. Thus $\beta$ satisfies the axiom $(\mathrm{C}-2)$. We have verified the axioms $(\mathrm{C}-1)$ and $(\mathrm{C}-2)$ for $\beta$, so $\beta$ is a $\widetilde{P}$-premotivic pseudonatural transformation.

Now, we will show the uniqueness of $\beta$. If $\beta^{\prime}: G \rightarrow G^{\prime}$ be another $\widetilde{P}$-premotivic pseudonatural transformation such that the diagram

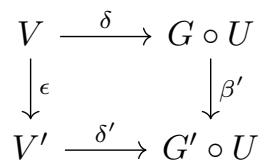

commutes, we have to show that there is an isomorphism $\Phi: \beta \rightarrow \beta^{\prime}$. We will construct it as follows. For any object $\widetilde{X_{1}}=\left(Z_{1} \stackrel{i_{1}}{\rightarrow} S_{1}\right)$ of $\widetilde{\mathcal{C}}$, put $\Phi_{\widetilde{X_{1}}}$ as the composition

$$
\begin{gathered}
\beta\left(\widetilde{X_{1}}\right) \stackrel{a d^{\prime-1}}{\longrightarrow} \beta\left(\widetilde{X_{1}}\right) \rho_{i_{1}}^{*} \rho_{i_{1} *} \stackrel{\beta_{\rho_{i_{1}}}}{\longrightarrow} \rho_{i_{1}}^{*} \beta\left(S_{1}\right) \rho_{i_{1} *} \stackrel{\sim}{\longrightarrow} \rho_{i_{1}}^{*} \epsilon\left(S_{1}\right) \rho_{i_{1} *} \\
\stackrel{\sim}{\longrightarrow} \rho_{i_{1}}^{*} \beta^{\prime}\left(S_{1}\right) \rho_{i_{1} *} \stackrel{\beta_{\rho_{i}}^{-1}}{\longrightarrow} \beta^{\prime}\left(\widetilde{X_{1}}\right) \rho_{i_{1}}^{*} \rho_{i_{1} *} \stackrel{a d^{\prime}}{\longrightarrow} \beta^{\prime}\left(\widetilde{X_{1}}\right) .
\end{gathered}
$$

Here, the first arrow is defined and an isomorphism by (Loc), and the sixth arrow is also an isomorphism by (Loc). Thus $\Phi_{\widetilde{X_{1}}}$ is an isomorphism. The axiom of modifications for $\Phi$ is that for any morphism $\widetilde{f}_{1}: \widetilde{X_{2}} \rightarrow \widetilde{X_{1}}$ in $\widetilde{\mathcal{C}}$ given by a commutative diagram

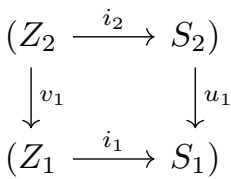

in $\mathcal{C}$, the diagram

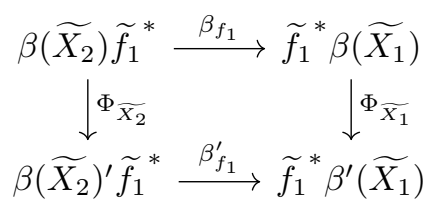


of functors commutes. This is true since the diagram is the big outside diagram of the diagram

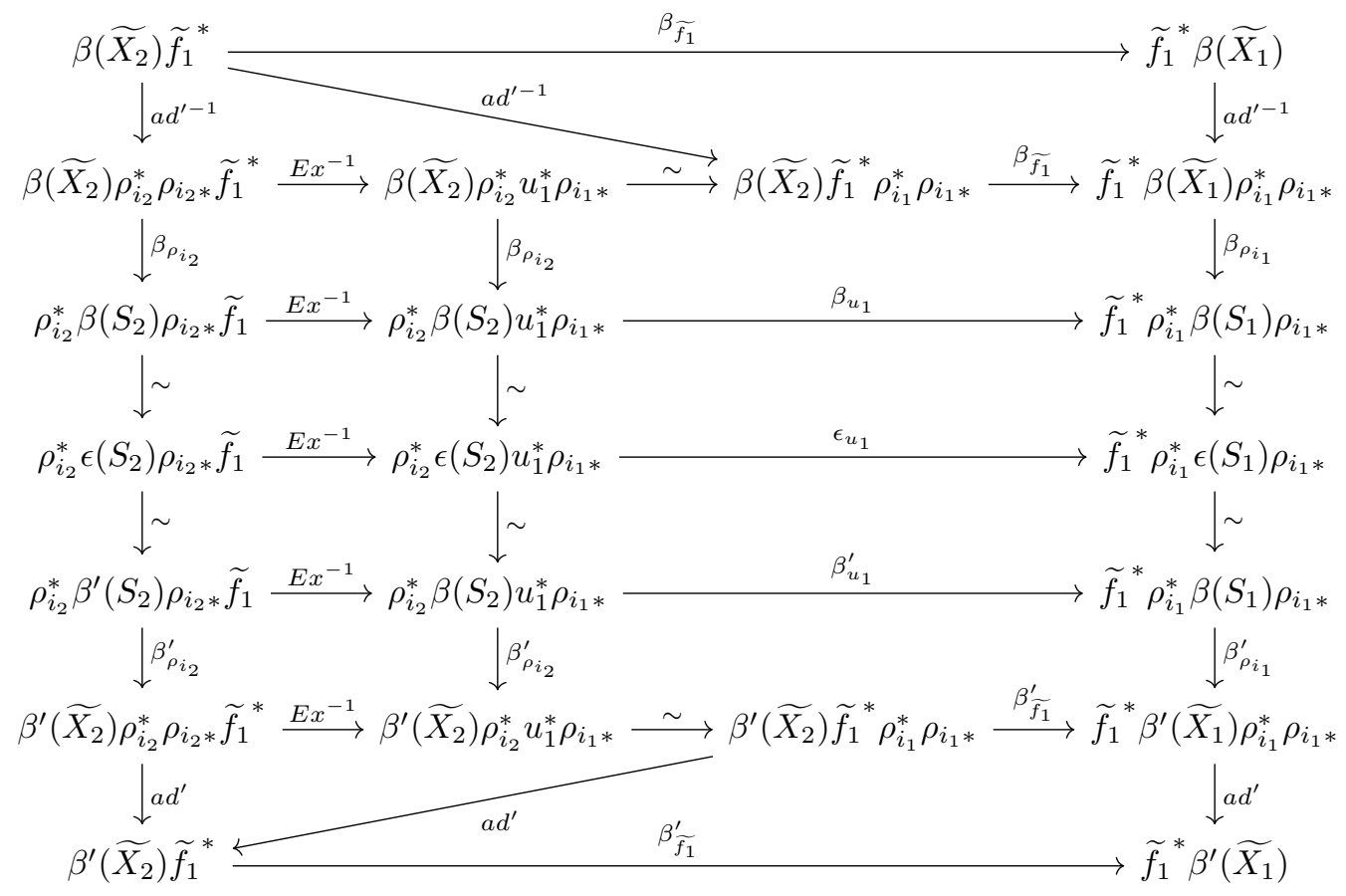

of functors, whose small diagrams commute. Here, the arrows denoted by $a d^{\prime-1}$ are defined and isomorphisms by (Loc), and the arrows denoted by $E x^{-1}$ are defined and an isomorphism by (4.10). Thus $\beta$ is isomorphic to $\beta^{\prime}$, so we have proved the uniqueness of $\beta$.

\section{Proof of (1.4), part III}

6.1. Under the notations and hypotheses of (4.4), assume $\mathcal{C}=\mathscr{S}$ and $\mathcal{E}=\mathscr{S}^{\text {sm }}$, and consider a commutative diagram

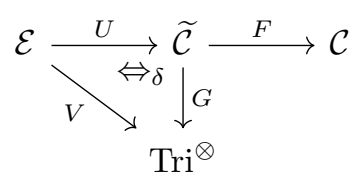

of 2-categories where

(i) $V$ is a $\mathscr{P}^{\prime}$-premotivic pseudofunctor satisfying (Loc).

(ii) $G$ is a $\widetilde{P}$-premotivic pseudofunctor satisfying (Loc),

(iii) $\delta$ is a pseudonatural equivalence. 
In this section, we will show that for any morphism $\widetilde{f_{1}}: \widetilde{X_{2}} \rightarrow \widetilde{X_{1}}$ given by a commutative diagram

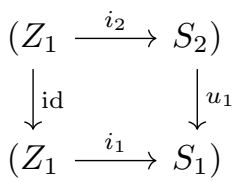

in $\mathcal{C}, \widetilde{f}_{1}^{*}$ is an equivalence.

Proposition 6.2. Under the notations and hypotheses of (6.1), if $u_{1}$ is a closed immersion, then $\widetilde{f}_{1}^{*}$ is an equivalence.

Proof. It suffices to show that the counit and unit

$$
\widetilde{f}_{1}^{*} \widetilde{f}_{1 *} \stackrel{a d^{\prime}}{\longrightarrow} \text { id, } \quad \text { id } \stackrel{a d}{\longrightarrow} \widetilde{f}_{1_{*}} \widetilde{f}_{1}^{*}
$$

are isomorphisms. The counit is the composition

$$
\rho_{i_{2}}^{*} u_{1}^{*} \rho_{i_{1} *} \rho_{i_{1}}^{!} u_{1 *} \rho_{i_{2} *} \stackrel{a d^{\prime}}{\longrightarrow} \rho_{i_{2}}^{*} u_{1}^{*} u_{1 *} \rho_{i_{2} *} \stackrel{a d^{\prime}}{\longrightarrow} \rho_{i_{2}}^{*} \rho_{i_{2} *} \stackrel{a d^{\prime}}{\longrightarrow} \text { id. }
$$

The first arrow is an isomorphism by (5.6(1)), and the second and third arrows are isomorphisms by (Loc). Thus the composition is also an isomorphism.

The unit id $\stackrel{a d}{\longrightarrow} \widetilde{f}_{1_{*}} \widetilde{f}_{1}^{*}$ is the composition

$$
\text { id } \stackrel{a d}{\longrightarrow} \rho_{i_{1}}^{!} \rho_{i_{1} *} \stackrel{a d}{\longrightarrow} \rho_{i_{1}}^{!} u_{1 *} u_{1}^{*} \rho_{i_{1} *} \stackrel{a d}{\longrightarrow} \rho_{i_{1}}^{!} u_{1_{*}} \rho_{i_{2} *} \rho_{i_{2}}^{*} u_{1}^{*} \rho_{i_{1} *} .
$$

The first arrow is an isomorphism by (Loc), and the third arrow is an isomorphism by (5.6(2)). Thus the remaining is to show that the second arrow is an isomorphism.

Consider the commutative diagram

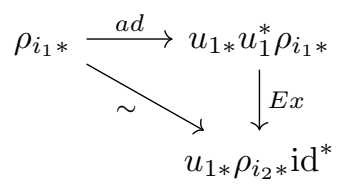

of functors. The vertical arrow is an isomorphism by (4.10), so the horizontal arrow is an isomorphism. Thus the second arrow of (6.2.1) is an isomorphism.

Proposition 6.3. Under the notations and hypotheses of (6.1), if $u_{1}$ is an open immersion, then $\widetilde{f}_{1}{ }^{*}$ is an equivalence.

Proof. It suffices to show that the counit and unit

$$
\widetilde{f}_{1}^{*} \widetilde{f}_{1_{*}} \stackrel{a d^{\prime}}{\longrightarrow} \text { id, } \quad \text { id } \stackrel{a d}{\longrightarrow} \widetilde{f}_{1_{*}} \widetilde{f}_{1}^{*}
$$

are isomorphisms. The counit is the composition

$$
\rho_{i_{2}}^{*} u_{1}^{*} \rho_{i_{1} *} \rho_{i_{1}}^{!} u_{1 *} \rho_{i_{2} *} \stackrel{a d^{\prime}}{\longrightarrow} \rho_{i_{2}}^{*} u_{1}^{*} u_{1 *} \rho_{i_{2} *} \stackrel{a d^{\prime}}{\longrightarrow} \rho_{i_{2}}^{*} \rho_{i_{2} *} \stackrel{a d^{\prime}}{\longrightarrow} \text { id. }
$$


The first arrow is an isomorphism by (5.6(1)), and the second arrow is an isomorphism by $4.8(5))$. The third arrow is also an isomorphism by (Loc). Thus the composition is an isomorphism.

The unit id $\stackrel{a d}{\longrightarrow} \widetilde{f}_{1_{*}} \widetilde{f}_{1}^{*}$ is the composition

$$
\mathrm{id} \stackrel{a d}{\longrightarrow} \rho_{i_{1}}^{!} \rho_{i_{1} *} \stackrel{a d}{\longrightarrow} \rho_{i_{1}}^{!} u_{1 *} u_{1}^{*} \rho_{i_{1} *} \stackrel{a d}{\longrightarrow} \rho_{i_{1}}^{!} u_{1 *} \rho_{i_{2} *} \rho_{i_{2}}^{*} u_{1}^{*} \rho_{i_{1} *} .
$$

The first arrow is an isomorphism by (Loc), and the third arrow is an isomorphism by (5.6(1)). Thus the remaining is to show that the second arrow is an isomorphism. For this, by definition, it suffices to show that the morphism

$$
K \stackrel{a d}{\longrightarrow} u_{1 *} u_{1}^{*} K
$$

in $V\left(S_{1}\right)$ is an isomorphism for any object $K$ of $V\left(S_{1}\right)$ such that $j_{1}^{*} K=0$. Here, $j_{1}$ denotes the complement of $i_{1}$.

Consider the Cartesian diagram

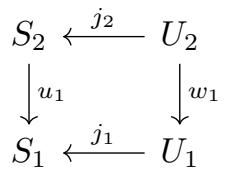

in $\mathcal{C}$. Then we have the Mayer-Vietoris distinguish triangle

$$
K \longrightarrow u_{1 *} u_{1}^{*} K \oplus j_{1 *} j_{1}^{*} K \longrightarrow\left(j_{1} w_{1}\right)_{*}\left(j_{1} w_{1}\right)^{*} K \longrightarrow K[1]
$$

in $V\left(S_{1}\right)$. Since $j_{1}^{*} K=0$, we have $\left(j_{1} w_{1}\right)^{*} K=0$. Thus the morphism $K \stackrel{a d}{\longrightarrow} u_{1 *} u_{1}^{*} K$ is an isomorphism.

Proposition 6.4. Under the notations and hypotheses of (6.1), the question that $\widetilde{f}_{1}^{*}$ is an equivalence is Zariski local on $S_{1}$.

Proof. Let $t_{1}: S_{1}^{\prime} \rightarrow S_{1}$ be a Zariski cover induced by open immersions

$$
j_{1}: U_{1} \rightarrow S_{1}, \ldots, j_{r}: U_{r} \rightarrow S_{1}
$$

and consider the diagram

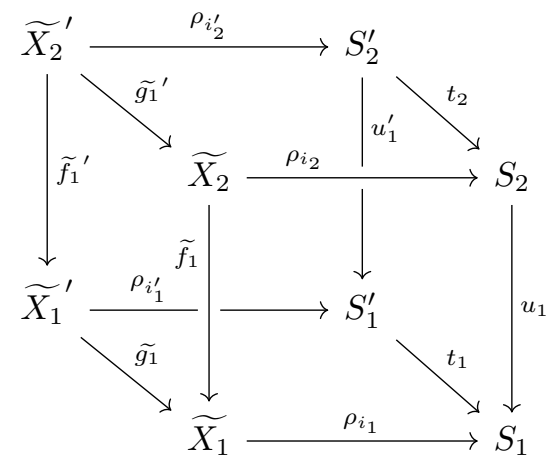

in $\widetilde{\mathcal{C}}$ where each square is Cartesian. The statement is that the equivalence of $\widetilde{f}_{1}^{\prime}$ implies the equivalence of $\widetilde{f}_{1}$. Hence assume that $\widetilde{f}_{1}^{\prime}$ is an equivalence. 
In the commutative diagrams
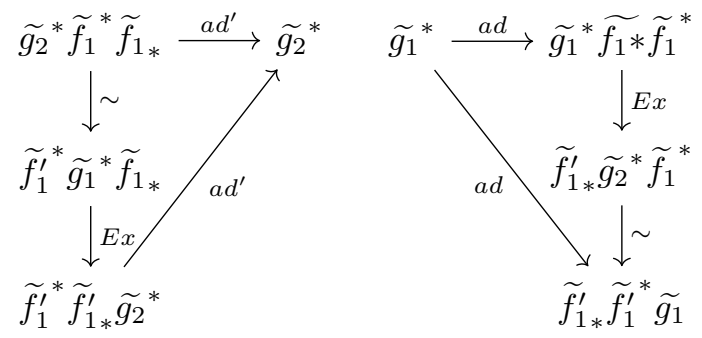

of functors, the diagonal arrows are isomorphisms since $\tilde{f}_{1}^{\prime}$ is an equivalence. The arrows denoted by $E x$ are also isomorphisms by (B-3). Thus the horizontal arrows are isomorphisms.

Then the natural transformations

$$
\rho_{i_{2}^{\prime} *}{\widetilde{g_{2}}}^{*} \widetilde{f}_{1}^{*} \widetilde{f}_{1_{*}} \stackrel{a d^{\prime}}{\longrightarrow} \rho_{i_{2}^{\prime}}{\widetilde{g_{2}}}^{*}, \quad \rho_{i_{1} *}^{\prime}{\widetilde{g_{1}}}^{*} \stackrel{a d}{\longrightarrow} \rho_{i_{1} *}^{\prime}{\widetilde{g_{1}}}^{*} \widetilde{f_{1} * \widetilde{f}_{1}}
$$

are isomorphisms, so by (4.10), the natural transformations

$$
t_{2}^{*} \rho_{i_{2} *} \widetilde{f}_{1}^{*} \widetilde{f}_{1_{*}} \stackrel{a d^{\prime}}{\longrightarrow} t_{2}^{*} \rho_{i_{2} *}, \quad t_{1}^{*} \rho_{i_{1} *} \stackrel{a d}{\longrightarrow} t_{1}^{*} \rho_{i_{1} *} \widetilde{f_{1} * \widetilde{f}_{1}^{*}}
$$

are isomorphisms. Then for any open immersion $j: U \rightarrow S_{1}$ that is an intersection of $j_{1}, \ldots, j_{n}$, the natural transformations

$$
j_{\sharp} j^{*} \rho_{i_{2} *} \widetilde{f}_{1}^{*} \widetilde{f}_{1_{*}} \stackrel{a d^{\prime}}{\longrightarrow} j_{\sharp} j^{*} \rho_{i_{2} *}, \quad j_{\sharp} j^{*} \rho_{i_{1} *} \stackrel{a d}{\longrightarrow} j_{\sharp} j^{*} \rho_{i_{1} *} \widetilde{f_{1} * \widetilde{f}_{1}^{*}}
$$

are isomorphisms. Applying the Mayer-Vietoris distinguished triangle repeatedly, we see that the natural transformations

$$
\rho_{i_{2} *}{\widetilde{f_{1}}}^{*} \widetilde{f}_{1 *} \stackrel{a d^{\prime}}{\longrightarrow} \rho_{i_{2} *}, \quad \rho_{i_{1} *} \stackrel{a d}{\longrightarrow} \rho_{i_{1} *} \widetilde{f_{1} * \widetilde{f}_{1}^{*}}
$$

are isomorphisms. Since $\rho_{i_{1} *}$ and $\rho_{i_{2} *}$ are fully faithful by (Loc), the counit and unit

$$
\widetilde{f}_{1}^{*} \widetilde{f}_{1 *} \stackrel{a d^{\prime}}{\longrightarrow} \text { id, } \quad \text { id } \stackrel{a d}{\longrightarrow} \widetilde{f_{1}} * \widetilde{f}_{1}^{*}
$$

are isomorphism. Thus $\widetilde{f}_{1}$ is an equivalence.

Proposition 6.5. Under the notations and hypotheses of (6.1), consider morphisms

$$
\widetilde{X_{2}} \underset{\tilde{f}_{2}}{\stackrel{\widetilde{f_{1}}}{\longrightarrow}} \widetilde{X_{1}}
$$

in $\widetilde{\mathcal{C}}$ given by a diagram

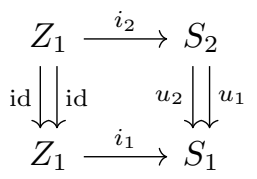

in $\mathcal{C}$. Then there is a natural isomorphism

$$
\widetilde{f}_{1}^{*} \cong{\widetilde{f_{2}}}^{*} .
$$


Proof. The morphisms $u_{1}$ and $u_{2}$ have the factorizations

$$
S_{2} \underset{\left(u_{2}, \text { id }\right)}{\stackrel{\left(u_{1}, \mathrm{id}\right)}{\longrightarrow}} S_{1} \times S_{2} \longrightarrow S_{1}
$$

where the right arrow is the projection. The left arrows are closed immersions since $S_{1}$ is separated. Hence replacing $S_{1}$ by $S_{1} \times S_{2}$, we may assume that $u_{1}$ and $u_{2}$ are closed immersions.

Then consider the diagram

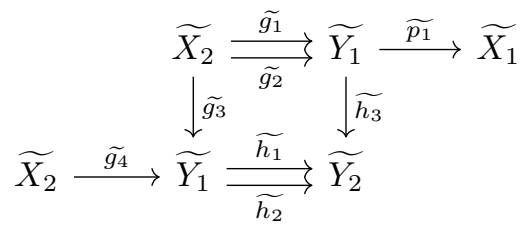

in $\widetilde{\mathcal{C}}$ given by the diagram

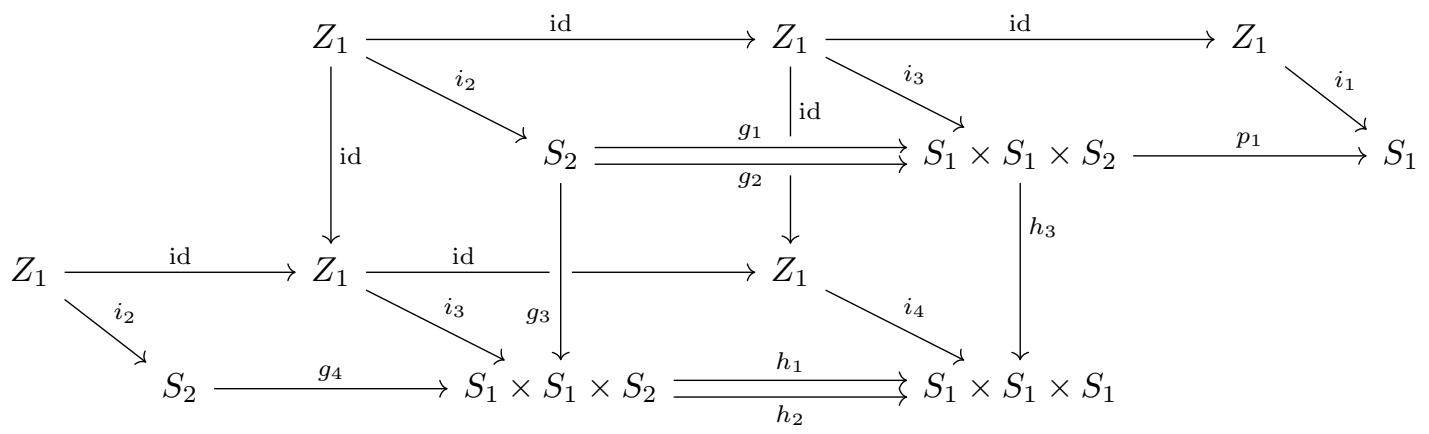

in $\mathcal{C}$ where

(i) $i_{3}=\left(i_{1}, i_{1}, i_{2}\right), i_{4}=\left(i_{1}, i_{1}, i_{1}\right)$,

(ii) $g_{1}=\left(u_{1}, u_{2}\right.$, id $), g_{2}=\left(u_{2}, u_{1}\right.$, id $), g_{3}=\left(u_{1}, u_{1}\right.$, id $), g_{4}=\left(u_{2}, u_{2}\right.$, id $)$,

(iii) $p_{1}$ denotes the first projection,

(iv) $h_{1}=\tau_{23} \circ\left(\mathrm{id} \times \mathrm{id} \times u_{2}\right), h_{2}=\tau_{13} \circ\left(\mathrm{id} \times \mathrm{id} \times u_{2}\right), h_{3}=\mathrm{id} \times \mathrm{id} \times u_{1}$ where $\tau_{i j}$ denotes the transposition of $i$-th and $j$-th $S_{1}$.

Note that $g_{4}$ is a closed immersion since $S_{1}$ is separated. We have the natural transformations

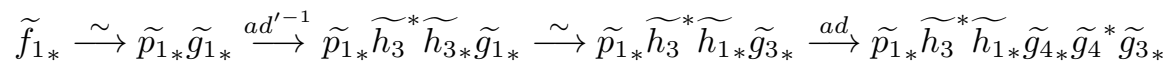

$$
\begin{aligned}
& \stackrel{\sim}{\longrightarrow} \widetilde{p_{1}}{\widetilde{h_{3}}}^{*}\left(\widetilde{h_{1}} \widetilde{g_{4}}\right)_{*} \widetilde{g}_{4}^{*} \widetilde{g}_{3 *} \stackrel{\sim}{\longrightarrow} \widetilde{p_{1}}{\widetilde{h_{3}}}^{*}\left(\widetilde{h_{2}} \widetilde{g_{4}}\right)_{*} \widetilde{g_{4}} * \widetilde{g_{3}} \stackrel{\sim}{\longrightarrow} \widetilde{p_{1}} \widetilde{h_{3}} * \widetilde{h_{2}} \widetilde{g_{4}} \widetilde{g}^{*} * \widetilde{g_{3}} \\
& \stackrel{a d^{-1}}{\longrightarrow} \widetilde{p_{1}} \widetilde{h_{3}}{ }^{*} \widetilde{h_{2}} \widetilde{g_{3}} \stackrel{\sim}{\longrightarrow} \widetilde{p_{1}} \widetilde{h_{3}} \widetilde{h}^{*} \widetilde{h_{3}} \widetilde{g_{2}} \stackrel{a d}{\longrightarrow} \widetilde{p_{1}} \widetilde{g_{2}} \stackrel{\sim}{\longrightarrow} \widetilde{f_{2}} \cdot
\end{aligned}
$$

Here, the second arrow is defined and an isomorphism by (Loc), and the tenth arrow is an isomorphism by the same reason. The fourth arrow is an isomorphism by (6.2) since $g_{4}$ is a closed immersion, and the eighth arrow is defined and an isomorphism by the same reason. Thus the composition is an isomorphism. Its left adjoint is $\widetilde{f}_{1}{ }^{*} \cong \widetilde{f}_{2}{ }^{*}$. 
Theorem 6.6. Under the notations and hypotheses of (6.1), $\widetilde{f}_{1}^{*}$ is an equivalence.

Proof. Consider the diagram

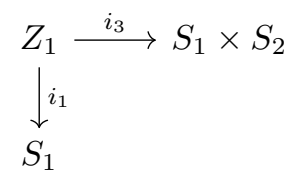

in $\mathcal{C}$ where $i_{3}$ denotes the morphism induced by $i_{1}$ and $i_{2}$. By [Sch05, 3.9], this can be extended to a Cartesian diagram

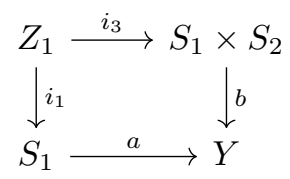

of schemes such that $a$ and $b$ are closed immersions. Choose a Zariski cover $\left\{Y_{i}\right\}_{i \in I}$ of $Y$ such that $I$ is finite and each $Y_{i}$ is affine, and put

$$
U_{i}=a^{-1}\left(Y_{i}\right), \quad V_{i}=q_{1}^{-1} b^{-1}\left(Y_{i}\right), \quad W_{i}=i_{1}^{-1}\left(U_{i}\right)
$$

where $q_{1}: S_{2} \rightarrow S_{1} \times S_{2}$ denotes the graph morphism of $u_{1}$. Then we have the commutative diagram

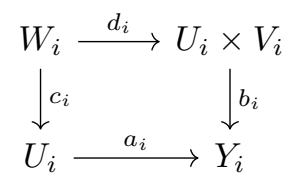

in $\mathcal{C}$ where $W_{i}=i_{1}^{-1}\left(U_{i}\right), c_{i}$ and $d_{i}$ are closed immersions induced by $i_{1}$ and $i_{2}$, and $a_{i}$ and $b_{i}$ are morphisms induced by $a$ and $b$. Note that $a_{i}$ is a closed immersion and that $b_{i}$ is an immersion.

The morphism $u_{1}: S_{2} \rightarrow S_{1}$ has the factorization

$$
S_{2} \stackrel{q_{1}}{\rightarrow} S_{1} \times S_{2} \stackrel{p_{7}}{\rightarrow} S_{1}
$$

where $p_{1}$ denotes the projection. Since $S_{1}$ is separated, $q_{1}$ is a closed immersion. Hence by (6.2), we reduce to the case when $\widetilde{f}_{1}$ is given by the commutative diagram

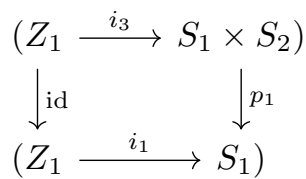

in $\mathcal{C}$. Then by (6.4), we reduce to the case when $\widetilde{f}_{1}$ is given by the commutative diagram

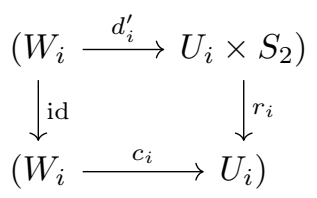


in $\mathcal{C}$ where $d_{i}^{\prime}$ denotes the morphism induced by $i_{3}$ and $r_{i}$ denotes the projection. Since $d_{i}^{\prime}$ has the factorization

$$
W_{i} \stackrel{d_{i}}{\rightarrow} U_{i} \times V_{i} \rightarrow U_{i} \times S_{2}
$$

where the second arrow is the open immersion induced by the open immersion $V_{i} \rightarrow S_{2}$, by (6.3), we reduce to the case when $\widetilde{f}_{1}$ is given by the commutative diagram

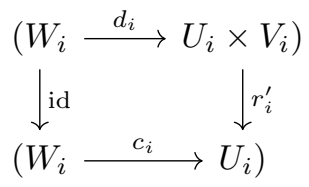

in $\mathcal{C}$ where $r_{i}^{\prime}$ denotes the projection. By (6.2), since $a_{i}$ is a closed immersion, we reduce to the case when $\widetilde{f}_{1}$ is given by the commutative diagram

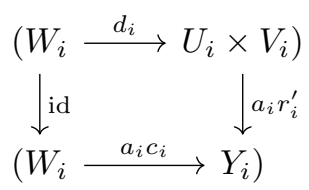

in $\mathcal{C}$. Consider the morphism $\widetilde{f}_{2}$ given by the commutative diagram

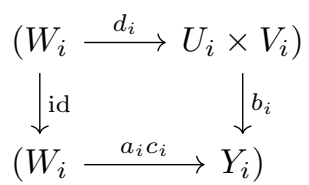

in $\mathcal{C}$. By (6.2) and (6.3), $\widetilde{f}_{2}^{*}$ is an equivalence since $b_{i}$ is an immersion, and by (6.5), $\widetilde{f}_{1}^{*} \cong \widetilde{f}_{2}^{*}$. Thus $\widetilde{f}_{1}^{*}$ is an equivalence.

\section{Proof of (1.4), part IV}

Theorem 7.1. Consider a commutative diagram

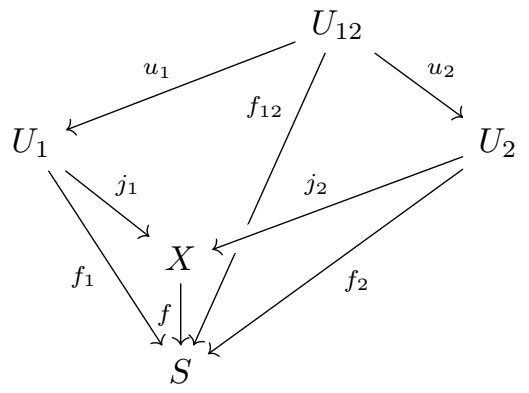

in $\mathscr{S}$ where the small inner square is Cartesian and $j_{1}$ and $j_{2}$ are open immersions. Let $\mathscr{P}$ be a class of morphisms in $\mathscr{S}$ containing all open immersions and stable by compositions and pullbacks, and let $H: \mathscr{S} \rightarrow \operatorname{Tri}^{\otimes}$ be a $\mathscr{P}$-motivic pseudofunctor satisfying (Loc). If $f_{1}^{*}, f_{2}^{*}$, and $f_{12}^{*}$ have left adjoints, then $f^{*}$ also has a left adjoint. 
Proof. Put $j_{12}=j_{1} u_{1}$, and we denote by $f_{1 \sharp}\left(\right.$ resp. $f_{2 \sharp}$, resp. $\left.f_{12 \sharp}\right)$ the left adjoint of $f_{1}^{*}$ (resp. $f_{2}^{*}$, resp. $\left.f_{12}^{*}\right)$.

(I) Construction of $f_{\sharp} K$. Consider the natural transformation

$$
\eta: f_{12 \sharp} j_{12}^{*} \longrightarrow f_{1 \sharp} j_{1}^{*} \oplus f_{2 \sharp} j_{2}^{*}
$$

given by the left adjoint of the composition

$$
\begin{aligned}
& j_{1 *} f_{1}^{*} \oplus j_{2 *} f_{2}^{*} \stackrel{\sim}{\longrightarrow} j_{1 *} j_{1}^{*} f^{*} \oplus j_{2 *} j_{2}^{*} f^{*} \\
& \stackrel{\operatorname{ad\oplus ad}}{\longrightarrow} j_{1 *} u_{1 *} u_{1}^{*} j_{1}^{*} f^{*} \oplus j_{2 *} u_{2 *} u_{2}^{*} j_{2}^{*} f^{*} \stackrel{\sim}{\longrightarrow} j_{12 *} f_{12}^{*} \oplus j_{12 *} f_{12}^{*} \longrightarrow j_{12 *} f_{12}^{*}
\end{aligned}
$$

where the fourth arrow is the summation. For any object $K$ of $H(X)$, using an axiom of triangulated categories, choose $f_{\sharp} K$ as a cone of $f_{12 \sharp} j_{12}^{*} \stackrel{\eta}{\longrightarrow} f_{1 \sharp} j_{1}^{*} \oplus f_{2 \sharp} j_{2}^{*}$. Then we have a distinguished triangle

$$
f_{12 \sharp} j_{12}^{*} K \stackrel{\eta}{\longrightarrow} f_{1 \sharp} j_{1}^{*} K \oplus f_{2 \sharp} j_{2}^{*} K \longrightarrow f_{\sharp} K \longrightarrow f_{12 \sharp} j_{12}^{*} K \longrightarrow f_{1 \sharp} j_{1}^{*}[1] .
$$

(II) Construction of $K \stackrel{a d}{\longrightarrow} f^{*} f_{\sharp} K$. Let $K$ be an object of $H(X)$. Consider the natural transformation

$$
\mu_{1}: j_{1 \sharp} \longrightarrow f^{*} f_{1 \sharp}
$$

given by the left adjoint of the composition

$$
f_{1}^{*} f_{*} \stackrel{\sim}{\longrightarrow} j_{1}^{*} f^{*} f_{*} \stackrel{a d^{\prime}}{\longrightarrow} j_{1}^{*} .
$$

We similarly have the natural transformations

$$
\begin{gathered}
\mu_{2}: j_{2 \sharp} \longrightarrow f^{*} f_{2 \sharp}, \\
\mu_{12}: j_{12 \sharp} \longrightarrow f^{*} f_{12 \sharp} .
\end{gathered}
$$

Then we have the commutative diagram

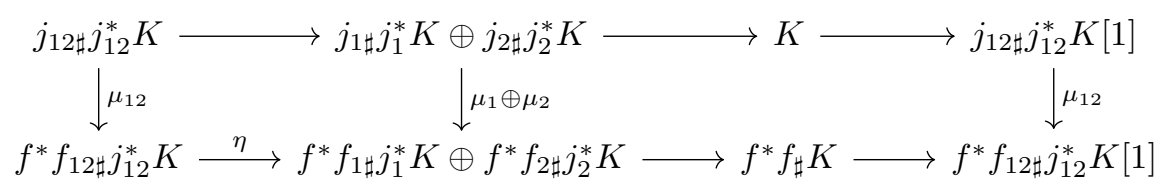

in $H(X)$ where the first row is the Mayer-Vietoris distinguished triangle and the second row is induced by the distinguished triangle (7.1.1). Using an axiom of triangulated categories, choose a morphism $K \stackrel{a d}{\longrightarrow} f^{*} f_{\sharp} K$ in $H(X)$ making the above diagram commutative.

(III) Construction of $\operatorname{Hom}_{H(S)}\left(f_{\sharp} K, L\right) \stackrel{\sim}{\longrightarrow} \operatorname{Hom}_{H(X)}\left(K, f^{*} L\right)$. Consider the homomorphism

$$
\varphi: \operatorname{Hom}_{H(S)}\left(f_{\sharp} K, L\right) \longrightarrow \operatorname{Hom}_{H(X)}\left(K, f^{*} L\right)
$$

of abelian groups given by

$$
\left(f_{\sharp} K \stackrel{\alpha}{\longrightarrow} L\right) \mapsto\left(K \stackrel{a d}{\longrightarrow} f^{*} f_{\sharp} K \stackrel{\alpha}{\longrightarrow} f^{*} L\right) .
$$


We will show that $\varphi$ is bijective. Using the Mayer-Vietoris distinguished triangle

$$
j_{12 \sharp} j_{12}^{*} K \longrightarrow j_{1 \sharp} j_{1}^{*} K \oplus j_{2 \sharp} j_{2}^{*} K \longrightarrow K \longrightarrow j_{12 \sharp j_{12}^{*}} K[1],
$$

we reduce to the case when $K$ is in the essential image of $j_{1 \sharp}, j_{2 \sharp}$, or $j_{12 \sharp}$. We will only consider the case when $K$ is in the essential image of $j_{1 \sharp}$ since the proofs for the other two cases are the same.

If $K=j_{1 \sharp} K^{\prime}$ for some object $K^{\prime}$ of $H\left(X_{1}\right)$, using [4.8(5)) to (7.1.1), we have the isomorphism

$$
\psi: f_{1 \sharp} K^{\prime} \longrightarrow f_{\sharp} j_{1 \sharp} K^{\prime} .
$$

Then using (4.8(5)) to (7.1.2), we have the commutative diagram

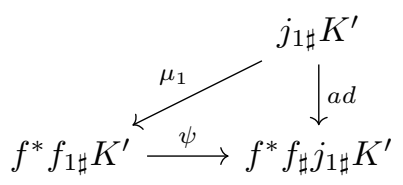

in $H(X)$.

Let $L$ be an object of $H(S)$, and consider the diagram

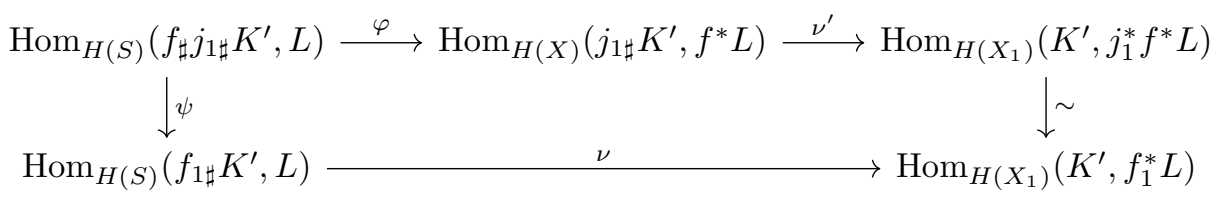

of abelian groups where $\nu$ (resp. $\nu^{\prime}$ ) denotes the isomorphisms induced by the adjunction of $f_{1 \sharp}$ and $f_{1}^{*}$ (resp. $j_{1 \sharp}$ and $\left.j_{1}^{*}\right)$. Then consider an element $\alpha \in \operatorname{Hom}_{H(S)}\left(f_{\sharp} j_{1 \sharp} K^{\prime}, L\right)$. Its image in $\operatorname{Hom}_{H\left(X_{1}\right)}\left(K^{\prime}, f_{1}^{*} L\right)$ via the upper right route and the lower left route are the compositions

$$
\begin{gathered}
K^{\prime} \stackrel{a d}{\longrightarrow} j_{1}^{*} j_{1 \sharp} K^{\prime} \stackrel{a d}{\longrightarrow} j_{1}^{*} f^{*} f_{\sharp} j_{1 \sharp} K^{\prime} \stackrel{\alpha}{\longrightarrow} j_{1}^{*} f^{*} L \stackrel{\sim}{\longrightarrow} f_{1}^{*} L, \\
K^{\prime} \stackrel{a d}{\longrightarrow} f_{1}^{*} f_{1 \sharp} K^{\prime} \stackrel{\psi^{-1}}{\longrightarrow} f_{1}^{*} f_{\sharp} j_{1 \sharp} K^{\prime} \stackrel{\alpha}{\longrightarrow} f_{1}^{*} L
\end{gathered}
$$

respectively. They are equal since the diagram

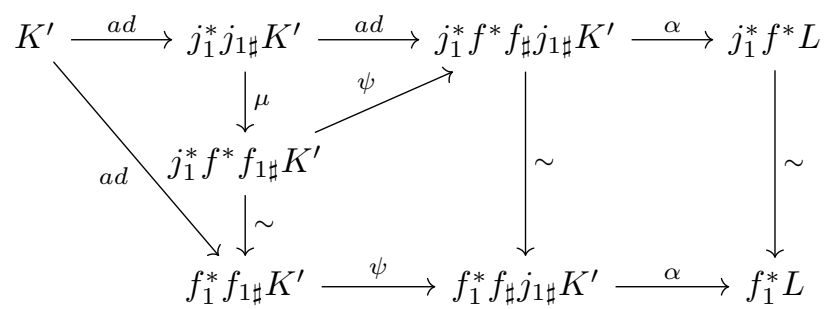

in $H(S)$ commutes by the commutativity of (7.1.3). Thus (7.1.4) commutes. This means that the homomorphism

$$
\varphi: \operatorname{Hom}_{H(S)}\left(f_{\sharp} K, L\right) \longrightarrow \operatorname{Hom}_{H(X)}\left(K, f^{*} L\right)
$$

is an isomorphism. 
(IV) Construction of $f_{\sharp} K \longrightarrow f_{\sharp} L$. Let $K$ and $L$ be objects of $H(X)$. By (III), the homomorphism

$$
\varphi: \operatorname{Hom}_{H(S)}\left(f_{\sharp} K, f_{\sharp} L\right) \longrightarrow \operatorname{Hom}_{H(X)}\left(K, f^{*} f_{\sharp} L\right)
$$

given by

$$
\left(f_{\sharp} K \stackrel{\alpha^{\prime}}{\longrightarrow} f_{\sharp} L\right) \mapsto\left(K \stackrel{a d}{\longrightarrow} f^{*} f_{\sharp} K \stackrel{\alpha^{\prime}}{\longrightarrow} f^{*} f_{\sharp} L\right)
$$

is an isomorphism. This means that for any morphism $\alpha: K \rightarrow L$ in $H(X)$, in the diagram

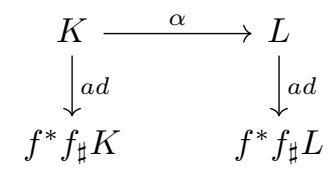

in $H(X)$, there is a unique morphism $f_{\sharp} K \longrightarrow f_{\sharp} L$ in $H(S)$ such that the induced morphism $f^{*} f_{\sharp} K \longrightarrow f^{*} f_{\sharp} L$ makes the above diagram commutative. This morphism is denoted by $f_{\sharp} \alpha$ : $f_{\sharp} K \rightarrow f_{\sharp} L$.

(V) Functoriality of $f_{\sharp}$. Let $\alpha: K \rightarrow L$ and $\beta: L \rightarrow M$ be morphisms in $H(S)$. Consider the diagram

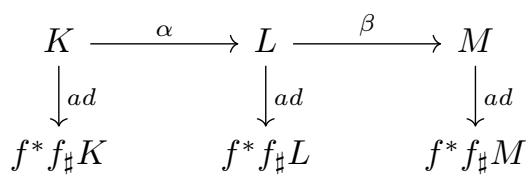

in $H(S)$. By definition, $f_{\sharp} \alpha$ (resp. $f_{\sharp} \beta$, resp. $f_{\sharp}(\beta \alpha)$ ) is a unique morphism such that the induced morphism $f^{*} f_{\sharp} K \longrightarrow f^{*} f_{\sharp} L$ (resp. $f^{*} f_{\sharp} K \longrightarrow f^{*} f_{\sharp} L$, resp. $f^{*} f_{\sharp} K \longrightarrow f^{*} f_{\sharp} M$ ) makes the above diagram commutative. Thus

$$
f_{\sharp} \beta \circ f_{\sharp} \alpha=f_{\sharp}(\beta \circ \alpha),
$$

so $f_{\sharp}$ is a functor.

(VI) Final step of the proof. We will show that $f_{\sharp}$ is left adjoint to $f^{*}$ to complete the proof. For this, it suffices to show that the isomorphism

$$
\varphi: \operatorname{Hom}_{H(S)}\left(f_{\sharp} K, L\right) \longrightarrow \operatorname{Hom}_{H(X)}\left(K, f^{*} L\right)
$$

is functorial on $K$ and $L$. Let $\beta: K \rightarrow K^{\prime}$ be a morphism in $H(X)$, and consider the diagram

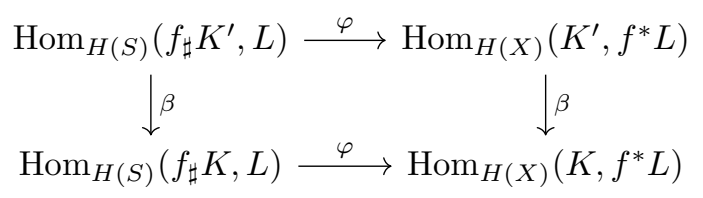

of abelian groups. For an element $\alpha \in \operatorname{Hom}_{H(S)}\left(f_{\sharp} K^{\prime}, L\right)$, its image in $\operatorname{Hom}_{H(X)}\left(K, f^{*} L\right)$ via the upper right route and lower left route are the compositions

$$
\begin{gathered}
K \stackrel{\beta}{\longrightarrow} K^{\prime} \stackrel{a d}{\longrightarrow} f^{*} f_{\sharp} K^{\prime} \stackrel{\alpha}{\longrightarrow} f^{*} L, \\
K \stackrel{a d}{\longrightarrow} f^{*} f_{\sharp} K \stackrel{\beta}{\longrightarrow} f^{*} f_{\sharp} K^{\prime} \stackrel{\alpha}{\longrightarrow} f^{*} L
\end{gathered}
$$


respectively. They are equal since the diagram

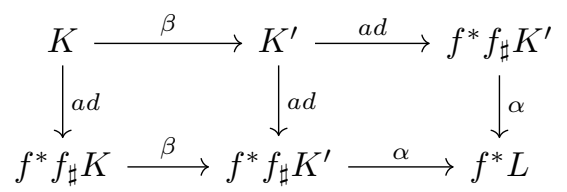

in $H(X)$ commutes. Thus (7.1.5) commutes, so $\varphi$ is functorial on $K$.

Let $\gamma: L \rightarrow L^{\prime}$ be a morphism in $H(X)$, and consider the diagram

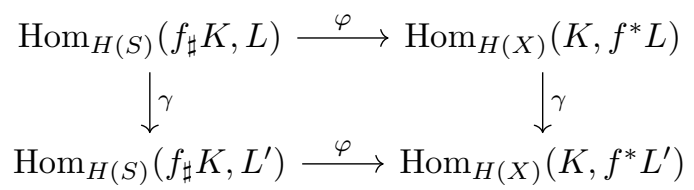

of abelian groups. For any element $\alpha \in \operatorname{Hom}_{H(S)}\left(f_{\sharp} K, L\right)$, its image in $\operatorname{Hom}_{H(X)}\left(K, f^{*} L^{\prime}\right)$ via the upper right route and lower left route are both equal to the composition

$$
K \stackrel{a d}{\longrightarrow} f^{*} f_{\sharp} K \stackrel{\alpha}{\longrightarrow} f^{*} L \stackrel{\beta}{\longrightarrow} f^{*} L^{\prime} .
$$

Thus (7.1.6) commutes, so $\varphi$ is functorial on $L$. This completes the proof that $f_{\sharp}$ is left adjoint to $f^{*}$.

Proposition 7.2. Under the notations and hypotheses of (7.1), let $H^{\prime}: \mathscr{S} \rightarrow \operatorname{Tri}^{\otimes}$ be another pseudofunctor satisfying (Loc), and let $\gamma: H \rightarrow H^{\prime}$ be a pseudonatural transformation satisfying $\left(\mathrm{C}_{-}\right.$ $1)$. Assume that $f^{*}, f_{1}^{*}, f_{2}^{*}$, and $f_{12}^{*}$ have left adjoints denoted by $f_{\sharp}, f_{1 \sharp}, f_{2 \sharp}$, and $f_{3 \sharp}$ respectively. If the exchange transformations

$$
f_{1 \sharp} \gamma\left(U_{1}\right) \stackrel{E x}{\longrightarrow} \gamma(S) f_{1 \sharp}, \quad f_{2 \sharp} \gamma\left(U_{2}\right) \stackrel{E x}{\longrightarrow} \gamma(S) f_{2 \sharp}, \quad f_{12 \sharp} \gamma\left(U_{12}\right) \stackrel{E x}{\longrightarrow} \gamma(S) f_{12 \sharp}
$$

are isomorphisms, then the exchange transformation

$$
f_{\sharp} \gamma(X) \stackrel{E x}{\longrightarrow} \gamma(S) f_{\sharp}
$$

is an isomorphism.

Proof. For each object $Y$ of $\mathcal{C}$, let $\gamma^{\prime}(Y)$ denote the right adjoint of $\gamma$. Consider the right adjoints of the above natural transformations

$$
f_{1}^{*} \gamma^{\prime}(S) \stackrel{E x}{\longrightarrow} \gamma^{\prime}\left(U_{1}\right) f_{1}^{*}, \quad f_{2}^{*} \gamma^{\prime}(S) \stackrel{E x}{\longrightarrow} \gamma^{\prime}\left(U_{2}\right) f_{2}^{*}, \quad f_{12}^{*} \gamma^{\prime}(S) \stackrel{E x}{\longrightarrow} \gamma^{\prime}\left(U_{1}\right) f_{12}^{*}, \quad f^{*} \gamma^{\prime}(S) \stackrel{E x}{\longrightarrow} \gamma^{\prime}(X) f^{*} .
$$

The first three natural transformations are isomorphisms by assumption. Consider the commutative diagram

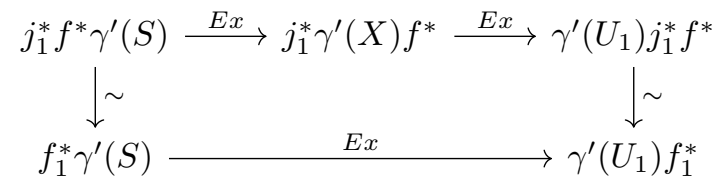


of functors. The lower horizontal arrow is an isomorphism by assumption, and the upper right horizontal arrow is an isomorphism since $\mathscr{P}$ contains all open immersions. Thus the upper left horizontal arrow is an isomorphism. Similarly we have isomorphisms

$$
j_{2}^{*} f^{*} \gamma^{\prime}(S) \stackrel{E x}{\longrightarrow} j_{2}^{*} \gamma^{\prime}(X) f^{*}, \quad j_{12}^{*} f^{*} \gamma^{\prime}(S) \stackrel{E x}{\longrightarrow} j_{12}^{*} \gamma^{\prime}(X) f^{*}
$$

are isomorphisms. Consider the commutative diagram

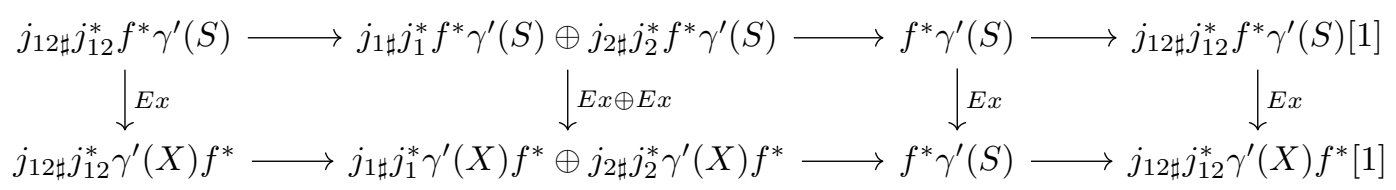

of functors where each row is induced by the Mayer-Vietoris distinguished triangle. The first and second columns are isomorphisms, so the third column is an isomorphism.

7.3. Now, we prove the main theorem (1.4).

Theorem 7.4. Consider a diagram

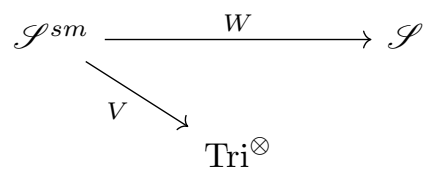

of 2-categories where

(i) $V$ is a Sm-premotivic pseudofunctor satisfying (Loc),

(ii) $W$ denotes the inclusion functor.

Then there is a contravariant pseudofunctor $H: \mathscr{S} \rightarrow$ Tri $^{\otimes}$ satisfying (Loc) and a pseudonatural equivalence $\kappa: V \rightarrow H \circ W$ making the above diagram commutes.

The above construction is functorial in the following sense. Suppose that we have a commutative diagram

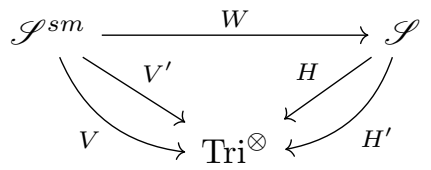

of 2-categories and a diagram

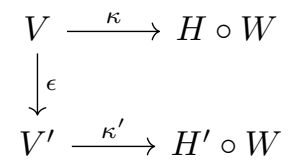

of pseudofunctors where $V^{\prime}$ and $H^{\prime}$ are Sm-premotivic pseudofunctors satisfying (Loc), $\kappa^{\prime}$ is a pseudonatural equivalence, and $\epsilon$ is a Sm-premotivic pseudonatural transformation. Then there exists a Sm-premotivic pseudonatural transformation $H \rightarrow H^{\prime}$ unique up to isomorphism such that the induced Sm-pseudonatural transformation $H \circ W \rightarrow H^{\prime} \circ W$ makes the diagram (1.4.1) commutative. 
Proof. Consider the diagram

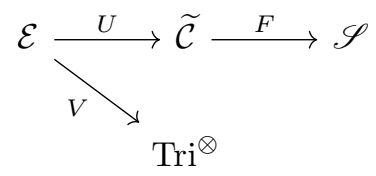

of 2-categories where $\mathcal{C}=\mathscr{S}, \mathcal{E}=\mathscr{S}^{s m}$, and $\widetilde{\mathcal{C}}, F$, and $U$ are given by (4.4). By (5.13), there are a $\widetilde{S m}$-premotivic pseudofunctor $G: \widetilde{\mathcal{C}} \rightarrow \operatorname{Tri}^{\otimes}$ satisfying (Loc) and a pseudonatural equivalence $\delta: V \rightarrow G \circ U$ such that the diagram

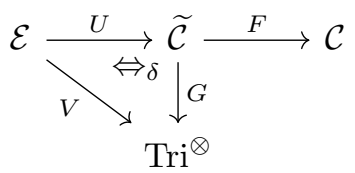

of 2-categories commutes. Then by (․6.6), for any morphism $\widetilde{f_{1}}: \widetilde{X_{2}} \rightarrow \widetilde{X_{1}}$ given by a commutative diagram

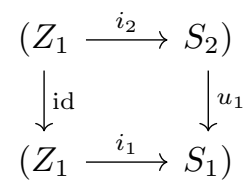

in $\mathcal{C}, \widetilde{f}_{1}{ }^{*}$ is an equivalence. Thus by (3.7), there are a contravariant pseudofunctor $H: \mathcal{C} \rightarrow \operatorname{Tri}^{\otimes}$ and a pseudonatural equivalence $\alpha: G \rightarrow H \circ F$ such that the diagram

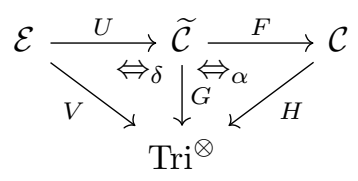

of 2-categories commutes, and by (2.13), $H$ is $F(\widetilde{S m})$-premotivic where $\widetilde{S m}$ is defined in (4.4). Thus we have a commutative diagram

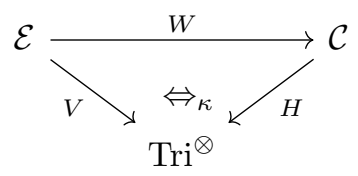

of 2-categories where $W=F \circ U$ and $\kappa=(\alpha \circ U) \circ \delta$. The remaining of the first part of the statement is showing that $H$ is $S m$-premotivic. For this, consider a smooth morphism $v_{1}: Z_{2} \rightarrow Z_{1}$ in $\mathcal{C}$, and choose a closed immersion $i_{1}: Z_{1} \rightarrow S_{1}$ where $S_{1}$ is in $\mathcal{E}$. Then by [EGA, IV.18.1.1], Zariski locally on $Z_{2}$, there is a Cartesian diagram

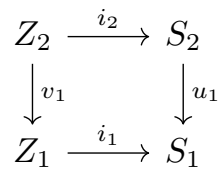


in $\mathcal{C}$ such that $u_{1}$ is smooth. Thus Zariski locally on $Z_{2}, v_{1}$ is a $F(\widetilde{S m})$-morphism, so Zariski locally on $Z_{1}, v_{1}^{*}$ has a left adjoint. Then (7.1) implies that $v_{1}$ has a left adjoint since $Z_{2}$ is quasi-compact. Thus we have proven the first part of the statement.

Consider a diagram

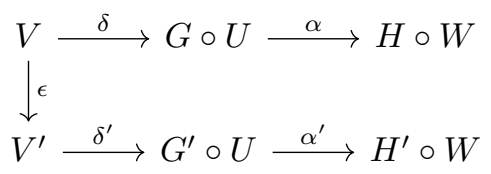

of pseudofunctors. By (5.15), there is a $\widetilde{S m}$-premotivic pseudonatural transformation $\beta: G \rightarrow G^{\prime}$ such that the induced pseudonatural transformation $G \circ U \rightarrow G^{\prime} \circ U$ makes the above diagram commutative, and then by (3.9) and (2.14), there is a $F(\widehat{S m})$-premotivic pseudonatural transformation $\gamma: H \rightarrow H^{\prime}$ such that the induced pseudonatural transformation $H \circ W \rightarrow H^{\prime} \circ W$ makes the above diagram commutes. By (7.2), $\gamma$ is $S m$-premotivic pseudonatural transformation as in the above paragraph. Hence the remaining to show that $\gamma$ is unique up to isomorphism. If $\gamma^{\prime}: H \rightarrow H^{\prime}$ is another $S m$-premotivic pseudonatural transformation such that the induced pseudonatural transformation $H \circ W \rightarrow H^{\prime} \circ W$ makes the above diagram commutative, then by (5.15), the two pseudonatural transformations

$$
\alpha^{\prime-1} \circ(\gamma \circ F) \circ \alpha: G \rightarrow G^{\prime}, \quad \alpha^{\prime-1} \circ\left(\gamma^{\prime} \circ F\right) \circ \alpha: G \rightarrow G^{\prime}
$$

are isomorphic. Thus by (3.9), $\gamma$ and $\gamma^{\prime}$ are isomorphic since we have the commutative diagrams

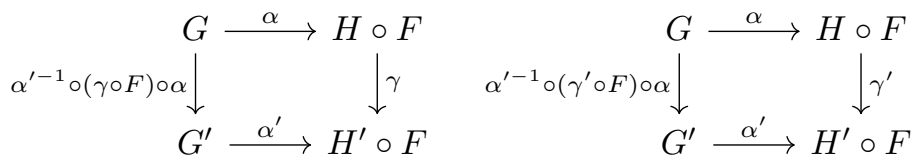

of pseudonatural transformations.

\section{Proof of (1.6)}

Theorem 8.1. Consider a commutative diagram

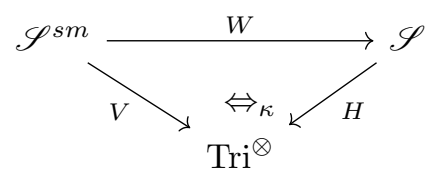

where

(i) $W$ denotes the inclusion functor,

(ii) $H$ is a $\mathscr{P}$-premotivic pseudofunctor satisfying (Loc),

(iii) $V$ is a $\mathscr{P}^{\prime}$-premotivic pseudofunctor satisfying (Loc),

(iv) $\kappa: V \rightarrow H \circ W$ is a pseudonatural equivalence.

Then we have the followings. 
(1) If $V$ satisfies (B-5), then $H$ satisfies (B-5).

(2) If $V$ satisfies (B-6), then $H$ satisfies (B-6).

(3) If $V$ satisfies (B-6) and (B-7), then $H$ satisfies (B-7).

(4) If $V$ satisfies (B-6) and (B-8), then $H$ satisfies (B-8).

Proof. (1) Let $i: Z \rightarrow S$ be a closed immersion in $\mathscr{S}$ where $S$ is in $\mathscr{S}^{s m}$. Consider the Cartesian diagram

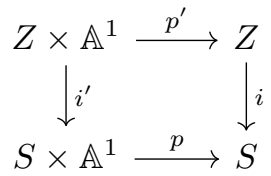

in $\mathscr{S}$ where $p$ denotes the projection. Then we have the commutative diagram

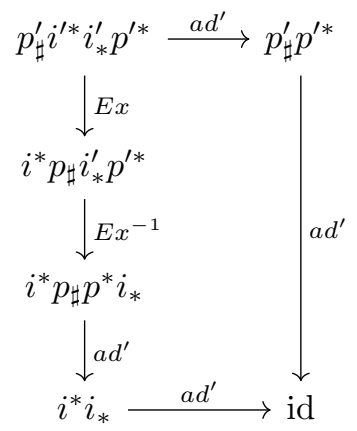

of functors. Here, the left middle vertical arrow is defined and an isomorphism by (4.10). The left top vertical arrow is an isomorphism by (B-3), and the lower horizontal and upper horizontal arrows are isomorphisms by (Loc). Thus the right vertical arrow is an isomorphism since the left bottom vertical arrow is an isomorphism by (B-5) for $V$.

(2) Let $i: Z \rightarrow S$ be a closed immersion in $\mathcal{C}$ such that $S \in \mathscr{S}^{s m}$. If $V$ satisfies (B-6), then there is an object $\tau$ of $H(S)$ such that $1_{S}(1) \otimes \tau \cong 1_{S}$. Then

$$
1_{Z}(1) \otimes i^{*} \tau \cong i^{*}\left(1_{S}(1) \otimes \tau\right) \cong i^{*} 1_{S} \cong 1_{Z},
$$

so $1_{Z}(1)$ is $\otimes$-invertible in $H(Z)$. Thus $H$ satisfies (B-6).

(3) Suppose that $V$ satisfies (B-6) and (B-7). Then by (2), $H$ satisfies (B-6). Let $i: Z \rightarrow S$ be a closed immersion in $\mathcal{C}$ such that $S \in \mathscr{S}^{s m}$. For any object $X$ of $\mathscr{S}$, let $\mathcal{F}_{X}$ denote the family consisting of objects

$$
f_{\sharp} 1_{X^{\prime}}(d)[n]
$$

in $H(S)$ where $(d, n) \in \mathbb{Z} \times \mathbb{Z}$ and $g: X^{\prime} \rightarrow X$ is a smooth morphism in $\mathscr{S}$. The remaining is to show that $\mathcal{F}_{Z}$ generates $H(Z)$. Let $L \rightarrow L^{\prime}$ be a morphism in $H(Z)$ such that the induced homomorphism

$$
\operatorname{Hom}_{H(Z)}\left(K^{\prime}, L\right) \rightarrow \operatorname{Hom}_{H(Z)}\left(K^{\prime}, L^{\prime}\right)
$$


is an isomorphism for any object $K^{\prime}$ of $\mathcal{F}_{Z}$. Then the induced homomorphism

$$
\operatorname{Hom}_{H(Z)}\left(i^{*} K, L\right) \rightarrow \operatorname{Hom}_{H(Z)}\left(i^{*} K, L^{\prime}\right)
$$

is an isomorphism for any object $K$ of $\mathcal{F}_{S}$, so the induced homomorphism

$$
\operatorname{Hom}_{H(S)}\left(K, i_{*} L\right) \rightarrow \operatorname{Hom}_{H(S)}\left(K, i_{*} L^{\prime}\right)
$$

is an isomorphism for any object $K$ of $\mathcal{F}_{S}$. Since $V$ satisfies (B-7), this means that the induced morphism $i_{*} L \rightarrow i_{*} L^{\prime}$ in $H(S)$ is an isomorphism. Thus the morphism $L \rightarrow L^{\prime}$ is an isomorphism since $i_{*}$ is fully faithful by (Loc). This proves that $H$ satisfies (B-7).

(4) Suppose that $V$ satisfies (B-6) and (B-8). Then by (2), $H$ satisfies (B-6). Let $i: Z \rightarrow S$ be a closed immersion in $\mathscr{S}$ such that $S$ is in $\mathscr{S}^{s m}$. We will first show that the object $1_{Z}$ in $H(Z)$ is compact. By (Loc), we have the distinguished triangle

$$
j_{\sharp} j^{*} 1_{S} \stackrel{a d^{\prime}}{\longrightarrow} 1_{S} \stackrel{a d}{\longrightarrow} i_{*} i^{*} 1_{S} \longrightarrow j_{\sharp} j^{*} 1_{S}[1]
$$

in $H(S)$. Since $V$ satisfies (B-8), $j_{\sharp} j^{*} 1_{S}$ and $1_{S}$ are compact. Thus $i_{*} i^{*} 1_{S}$ is compact, so $1_{Z} \cong i^{*} 1_{S}$ is compact since $i_{*}$ is fully faithful by (Loc). Let $g: Z^{\prime} \rightarrow Z$ be a smooth morphism in $\mathscr{S}$. Then we have

$$
\operatorname{Hom}_{H(Z)}\left(g_{\sharp} 1_{Z^{\prime}},-\right)=\operatorname{Hom}_{H\left(Z^{\prime}\right)}\left(1_{Z^{\prime}}, g^{*}(-)\right),
$$

and it commutes with small sums since $g^{*}$ commutes with small sums and we have shown that $1_{Z^{\prime}}$ is compact.

Theorem 8.2. In (7.4), if $V$ satisfies the axioms from (B-5) to (B-8), then $H$ satisfies the Grothendieck six operations formalism in (1.6).

Proof. It follows from (8.1), (4.15), and (4.16).

\section{Applications}

9.1. Consider the pseudofunctor

$$
\operatorname{DM}(-, \Lambda): \mathscr{S}^{s m} \rightarrow \operatorname{Tri}^{\otimes},
$$

which is the restriction of the pseudofunctor $\operatorname{DM}(-, \Lambda)$ to $\mathscr{S}^{s m}$ defined in [CD12, 11.1.1]. By CD12, 11.1.2], it satisfies the axioms from (B-1) to (B-6), and by [CD12, 11.1.6], it satisfies (B-7) and (B-8). Moreover, by [CD12, 11.4.2], it satisfies $\left(\operatorname{Loc}_{i}\right)$ for any closed immersion $i: Z \rightarrow S$ in $\mathscr{S}^{s m}$. Since $\operatorname{DM}(\emptyset, \Lambda)=0$, the pseudofunctor $\operatorname{DM}(-, \Lambda)$ satisfies (Loc). Thus by (7.4), we can define the following.

Definition 9.2. We denote by

$$
\operatorname{DM}^{l o c}(-, \Lambda): \mathscr{S} \rightarrow \operatorname{Tri}^{\otimes}
$$

a $S m$-premotivic pseudofunctor satisfying (Loc) that is an extension of $\operatorname{DM}(-, \Lambda): \mathscr{S}^{s m} \rightarrow \operatorname{Tri}^{\otimes}$ to $\mathscr{S}$, which is unique up to pseudonatural equivalence.

9.3. By (8.2), it satisfies the Grothendieck six operations formalism in [CD12, 2.4.50]. 
Remark 9.4. Note that the definition of $\operatorname{DM}^{l o c}(-, \Lambda)$ depends on the base scheme $T$. We usually assume that $T$ is the spectrum of a field or a Dedekind domain. When $T$ is changed by a smooth quasi-projective morphism $T^{\prime} \rightarrow T$ of schemes, by definition, $D M^{l o c}(-, \Lambda)$ is not changed.

9.5. We can also apply (7.4) to étale realization. Assume that $T$ is a noetherian scheme separated over Spec $\mathbb{Z}[1 / n]$ where $n>1$ is an integer. Consider the pseudofunctor

$$
\mathrm{DM}_{\grave{e} t}(-, \Lambda): \mathscr{S}^{s m} \rightarrow \operatorname{Tr}^{\otimes}
$$

defined in [CD16, 2.2.4]. Then consider the change of coefficients

$$
\operatorname{DM}(-, \mathbb{Z}) \rightarrow \operatorname{DM}(-, \mathbb{Z} / n \mathbb{Z}),
$$

which is the Nisnevich version of the $S m$-premotivic pseudonatural transformation in [CD16, 5.3.1]. Consider also the $S m$-premotivic pseudonatural transformation

$$
\mathrm{DM}(-, \mathbb{Z} / n \mathbb{Z}) \rightarrow \mathrm{DM}_{\grave{e} t}(-, \mathbb{Z} / n \mathbb{Z})
$$

defined in [CD16, 2.2.8]. We also have the $S m$-premotivic pseudonatural equivalence

$$
\mathrm{DM}_{\grave{e} t}(-, \mathbb{Z} / n \mathbb{Z}) \rightarrow \mathrm{D}_{\grave{e} t}(-, \mathbb{Z} / n \mathbb{Z})
$$

by [CD16, 4.4.5]. Here, for $S \in \mathscr{S}, \mathrm{D}_{\grave{e} t}(S, \mathbb{Z} / n \mathbb{Z})$ denotes the derived category of sheaves of $\mathbb{Z} / n \mathbb{Z}$ modules on the small étale site of $S$.

Then we obtain the étale realization $R_{\grave{e} t, n}$, which is the composition

$$
\operatorname{DM}(-, \mathbb{Z}) \rightarrow \operatorname{DM}(-, \mathbb{Z} / n \mathbb{Z}) \rightarrow \mathrm{DM}_{\grave{e ̀ t}}(-, \mathbb{Z} / n \mathbb{Z}) \rightarrow \mathrm{D}_{\grave{e} t}(-, \mathbb{Z} / n \mathbb{Z}) .
$$

The pseudofunctor

$$
\mathrm{D}_{\grave{e} t}(-, \mathbb{Z} / n \mathbb{Z}): \mathscr{S} \rightarrow \operatorname{Tri}^{\otimes}
$$

satisfies (Loc) and the axioms from (B-1) to (B-4), whose proofs are in [SGA4, tome 3]. Hence by (7.4), we can define the following.

Definition 9.6. Assume that $T$ is a noetherian scheme separated over $\operatorname{Spec} \mathbf{Z}[1 / n]$ where $n>1$ is an integer. We denote by

$$
R_{\grave{e ̀ t}, n}^{l o c}: \mathrm{DM}^{l o c}(-, \mathbb{Z}) \rightarrow \mathrm{D}_{\grave{e} t}(-, \mathbb{Z} / n \mathbb{Z})
$$

a $S m$-premotivic pseudonatural transformation that is an extension of $R_{\grave{e} t, n}$ to $\mathscr{S}$, which is unique up to isomorphism.

Remark 9.7. Note that the remark (9.4) is also applied for $R_{\grave{e} t, n}^{l o c}$.

9.8. Using (9.6), we can also obtain the $\ell$-adic realization

$$
\mathrm{DM}^{l o c}(-, \mathbb{Z}) \rightarrow \mathrm{D}_{\grave{e} t}\left(-, \mathbb{Z}_{\ell}\right)
$$

when $T$ is a noetherian scheme separated over $\operatorname{Spec} \mathbb{Z}[1 / \ell]$.

9.9. In the case that $\Lambda$ is a $\mathbf{Q}$-algebra, let us compare $\operatorname{DM}^{l o c}(-, \Lambda)$ with $\mathrm{DA}_{\grave{e} t}(-, \Lambda$ ) (see Ayo14, 3.1] for the definition of $\mathrm{DA}_{t}(-, \Lambda)$ where $t$ is a topology on $\left.\mathscr{S}\right)$ when $T$ is the spectrum of a field or a Dedekind domain. They are equivalent on $\mathscr{S}^{s m}$ by [CD12, 16.2.22], and the pseudofunctor

$$
\operatorname{DA}(-, \Lambda): \mathscr{S} \rightarrow \operatorname{Tri}^{\otimes}
$$

satisfies (Loc) by CD12, 6.2.2]. Thus by (7.4), there is a pseudonatural equivalence between $\mathrm{DM}^{l o c}(-, \Lambda): \mathscr{S} \rightarrow \operatorname{Tri}^{\otimes}$ and $\mathrm{DA}_{\grave{e} t}(-, \Lambda): \mathscr{S} \rightarrow \operatorname{Tri}^{\otimes}$ that is an extension of the pseudonatural equivalence on $\mathscr{S}^{s m}$, which is unique up to isomorphism. 


\section{Orientation}

10.1. Consider the $S m$-premotivic pseudofunctor

$$
\operatorname{DM}^{e f f}(-, \Lambda): \mathscr{S}^{s m} \rightarrow \operatorname{Tri}^{\otimes},
$$

which is the restriction of the $S m$-premotivic pseudofunctor $\operatorname{DM}^{e f f}(-, \Lambda)$ defined in [CD12, 11.1.1]. By [CD12, 6.3.15], it satisfies $\left(\operatorname{Loc}_{i}\right)$ for any closed immersion $i: Z \rightarrow S$ in $\mathscr{S}^{s m}$. Then $\operatorname{DM}^{\text {eff }}(-, \Lambda)$ satisfies (Loc) since $\operatorname{DM}^{e f f}(\emptyset, \Lambda)=0$. Thus by (7.4), we can define the following.

Definition 10.2. We denote by

$$
\operatorname{DM}^{e f f, l o c}(-, \Lambda): \mathscr{S} \rightarrow \operatorname{Tri}^{\otimes}
$$

a $S m$-premotivic pseudofunctor satisfying (Loc) that is an extension of $\mathrm{DM}^{e f f}(-, \Lambda): \mathscr{S}^{s m} \rightarrow \operatorname{Tri}^{\otimes}$ to $\mathscr{S}$, which is unique up to pseudonatural equivalence.

10.3. Consider the $S m$-premotivic pseudofunctor

$$
\mathcal{H}_{\bullet}: \mathscr{S} \rightarrow \operatorname{Tri}^{\otimes}
$$

in [MV, Following paragraph of 3.2.12]. In [CD12, 11.2.16], if we use [CD12, 5.3.19] instead of [CD12, 5.3.28], we obtain the $S m$-premotivic pseudonatural transformation

$$
\mathrm{DA}_{N i s}(-, \Lambda) \rightarrow \mathrm{DM}^{e f f}(-, \Lambda)
$$

of pseudofunctors from $\mathscr{S}^{s m}$ to $\operatorname{Tri}^{\otimes}$. By the argument in [CD12, 5.3.35], we obtain the $S m$ premotivic pseudonatural transformation

$$
\mathcal{H}_{\bullet} \rightarrow \mathrm{DA}_{N i s}(-\Lambda)
$$

of pseudofunctors from $\mathscr{S}^{s m}$ to $\operatorname{Tri}^{\otimes}$. Thus by composing these two, we obtain the $S m$-premotivic pseudonatural transformation

$$
\mathcal{H}_{\bullet} \rightarrow \operatorname{DM}^{e f f}(-, \Lambda) \text {. }
$$

of pseudofunctors from $\mathscr{S}^{s m}$ to $\operatorname{Tri}^{\otimes}$ By [MV] 3.2.21], $\mathcal{H}_{\bullet}$ satisfies (Loc). Thus by (7.4), the above one can be extended to a $S m$-premotivic pseudonatural transformation

$$
\mathcal{H}_{\bullet} \rightarrow \mathrm{DM}^{\text {eff }, l o c}(-, \Lambda)
$$

of pseudofunctors from $\mathscr{S}$ to $\operatorname{Tr}^{\otimes}{ }^{\otimes}$, and it is unique up to isomorphism.

Consider the $S m$-premotivic pseudonatural transformation

$$
\Sigma^{\infty}: \mathrm{DM}^{e f f}(-, \Lambda) \rightarrow \mathrm{DM}(-, \Lambda)
$$

of pseudofunctors from $\mathscr{S}^{s m}$ to $\operatorname{Tri}^{\otimes}$ that is the restriction of $\Sigma^{\infty}$ to $\mathscr{S}^{s m}$ defined in CD12, 11.1.2.1]. Since $\mathrm{DM}^{\text {eff, loc }}$ satisfies (Loc), by (17.4), the above one can be extended to a $S m$-premotivic pseudonatural transformation

$$
\Sigma^{\infty}: \mathrm{DM}^{e f f, l o c}(-, \Lambda) \rightarrow \mathrm{DM}^{l o c}(-, \Lambda)
$$


of pseudofunctors from $\mathscr{S}$ to $\operatorname{Tr}^{\otimes}{ }^{\otimes}$, which is unique up to isomorphism.

Now in CD12, 11.3.1], we have the morphism $\mathfrak{c}_{1}: M\left(\mathbf{P}^{\infty}\right) \rightarrow \mathbf{1}(1)[2]$ in $\mathrm{DM}^{\text {eff }}(\operatorname{Spec} \mathbf{Z}, \Lambda)=$ $\mathrm{DM}^{e f f, l o c}(\operatorname{Spec} \mathbf{Z}, \Lambda)$. Then using the $S m$-premotivic pseudonatural transformations constructed in the above paragraphs, the argument in [CD12, §11.3] holds for $\operatorname{DM}^{e f f, l o c}(-, \Lambda)$ and $\operatorname{DM}^{l o c}(-, \Lambda)$. Thus we can construct a function

$$
c_{1}: \operatorname{Pic}(X) \rightarrow \operatorname{Hom}_{\mathrm{DM}^{l o c}(X, \Lambda)}(M(X), \mathbf{1}(1)[2])
$$

for each object $X$ of $\mathscr{S}$ such that $c_{1}$ is functorial on $X$ and that $c_{1}(L): M\left(\mathbf{P}^{1}\right) \rightarrow \mathbf{1}(1)[2]$ is the canonical projection where $L$ is the canonical bundle of $\mathbf{P}^{1}$.

Then by the argument in [CD12, 2.4.40], we have the following theorem.

Theorem 10.4. The Sm-premotivic pseudofunctor $\operatorname{DM}^{l o c}(-, \Lambda)$ admits an orientation in the sense of [CD12, 2.4.38].

\section{REFERENCES}

[Ayo07] J. Ayoub, Les six opérations de Grothendieck et le formalisme des cycles évanescents dans le monde motivique, Astérisque, vol. 314 and 315 (2007).

[Ayo14] J. Ayoub, La réalisation étale et les opérations de Grothendieck, Annales scientifiques de l'Ecole normale suprieure 47, fascicule 1 (2014) 1-145.

[CD12] D.-C. Cisinski and F. Déglise, Triangulated categories of mixed motives, arXiv:0912.2110v3, 2012.

[CD16] D.-C. Cisinski and F. Déglise, Étale motives, Compositio Mathematica, 152 (2016) no. 3 556-666.

[EGA] J. Dieudonn'e and A. Grothendieck. - Éléments de géométrie algébrique. Inst. Hautes Études Sci. Publ. Math. 4, 8, 11, 17, 20, 24, 28, 32, (1961-1967).

[MV] F. Morel and V. Voevodsky, A ${ }^{1}$-homotopy theory of schemes, Publ. Math. IHES 90 (1999), 45143.

[Sch05] K. Schwede, Gluing schemes and a scheme without closed points, In Recent progress in arithmetic and algebraic geometry, Contemp. Math. 386, Amer. Math. Soc., Providence, RI (2005) 157-172.

[SGA4] M. Artin, A. Grothendieck, and J.-L. Verdier, Théorie des topos et cohomologie étale des schémas, Lecture Notes in Mathematics 269, 270, 305, Springer-Verlag, Berlin (1972). 